\title{
NUCLEATE POOL BOILING HEAT TRANSFER IN WICKLESS HEAT PIPES (TWO-PHASE CLOSED THERMOSYPHONS): A CRITICAL REVIEW OF CORRELATIONS
}

\author{
Valentin Guichet ${ }^{1}$, Sulaiman Almahmoud ${ }^{1}$ and Hussam Jouhara ${ }^{1,{ }^{, *}}$ \\ 1. Institute of Energy Futures, College of Engineering, Design and Physical Sciences, \\ Brunel University London, UB8 3PH, UK \\ *. Corresponding author. E-mail address: hussam.jouhara@brunel.ac.uk (H. Jouhara)
}

\begin{abstract}
With the objective of estimating the pool boiling resistance of two-phase closed thermosyphons (TPCTs), the work presented gives the state of the art of nucleate pool boiling correlations. A deep analysis of up-to-date equations reported for bubbles and nucleate boiling is carried out and recommendations are provided to select the most suitable and reliable mathematical models. After introducing the operation of thermosyphons, a section dedicated to bubbles provides basic knowledge on nucleation processes and bubble growth. The main boiling mechanisms occurring in thermosyphons with a filling ratio of $100 \%$, i.e. pool boiling, are explained in a comprehensive way. The current state of the art of correlations predicting the number of active nucleation sites, the bubble departure diameters and bubble departure frequencies is presented. In addition, 27 correlations of nucleate pool boiling heat transfer coefficients are critically reviewed. For all sections, the advised correlations are reported in a clear and simple way using tables. The aim of this paper is to provide sufficient knowledge on twophase heat transfer in TPCTs to facilitate the nucleate pool boiling heat transfer coefficient calculations for thermosyphon users. This paper can be taken as a starting point in the study of pool boiling in thermosyphons and heat pipes.
\end{abstract}

Keywords: Boiling, Thermosyphons, Correlation, Heat transfer, Heat pipes

\section{Contents}

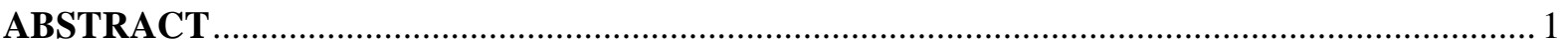

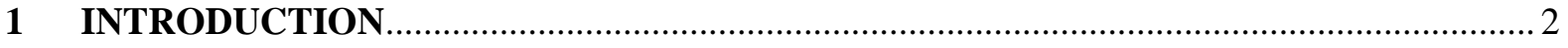

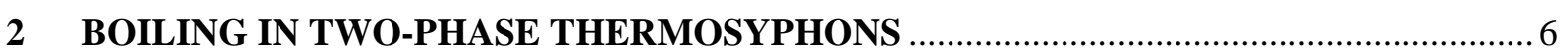

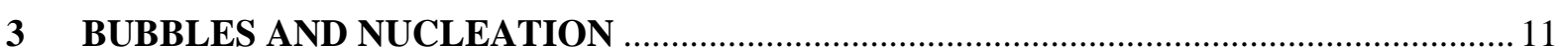

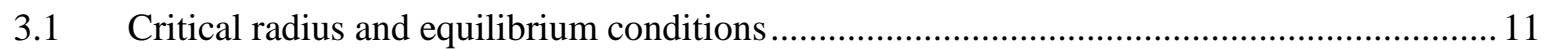

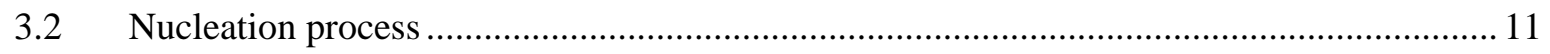

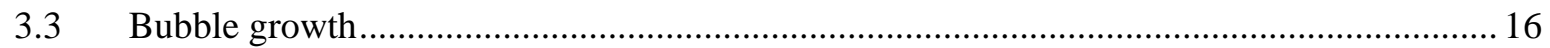

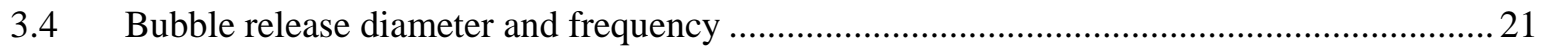

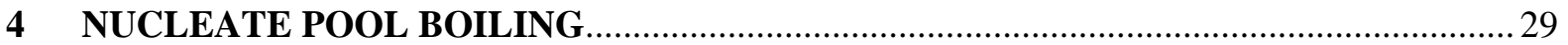

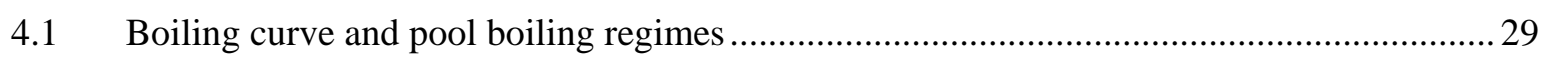

4.2 Nucleate pool boiling heat transfer correlations .................................................................. 32

5 FURTHER INVESTIGATIONS ON NUCLEATE POOL BOILING ….............................. 49

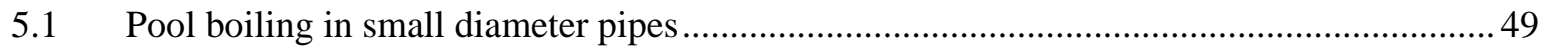

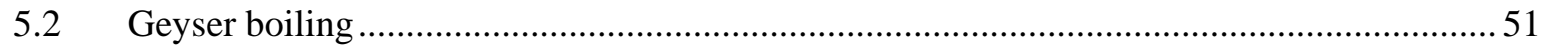

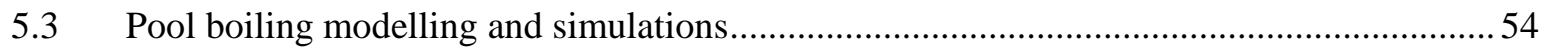




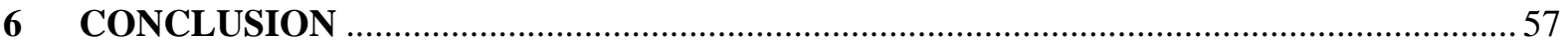

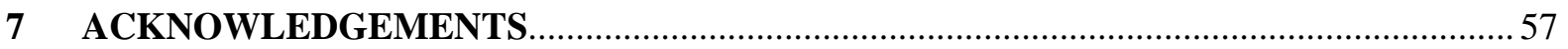

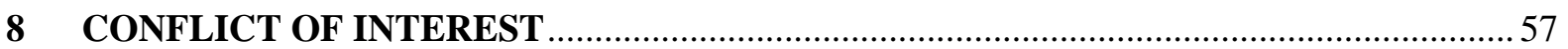

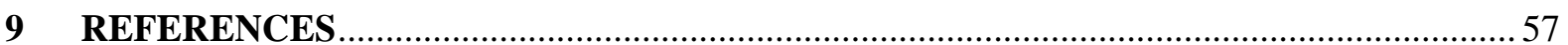

\section{INTRODUCTION}

First introduced by Gaugler [1] in 1944, heat pipes are passive devices capable of transmitting large amounts of heat with small temperature differences. By using a phase change cycle of a working fluid, the effective thermal conductivity of heat pipes can reach $100 \mathrm{~kW} \cdot \mathrm{m}^{-1} \mathrm{~K}^{-1}$ which is about 250 times higher than that of copper. In heat pipes, the working fluid receiving heat boils and turns to vapour at the evaporator section. Attracted by the temperature gradient in the pipe, the vapour condenses at the cold section of the condenser, thus releasing its heat by conduction. Finally, the liquid phase of the fluid returns to the evaporator by gravity (thermosyphons) or capillary action (wicked heat pipes). Heat pipes are not only attractive because of their high thermal conductivities; the isothermal surface property, the heat source and heat sink separation and the high reliability of heat pipes permit a wide range of applications [2]. Despite their early introduction, heat pipes were only investigated more deeply for industrial applications in the 1960s when the physicist George Grover developed in 1963 a capillarybased heat pipe to remove heat from space reactors without an external pump [3]. Following space applications, heat pipes were spread as cooling devices for CPUs and computers. Today, heat pipes are used in many sectors such as residential and commercial refrigeration systems, heating, ventilation and air conditioning (HVAC), waste-heat recovery, heat exchangers, data-centre cooling, metal and automotive industries, solar thermal and photovoltaic devices, geothermal energy, the food chain, nuclear and cryogenic systems, among others [2], [4]-[15]. With regard to the global warming concerns raised in the last decades, heat pipes are one of the environmentally-friendly solutions that open new opportunities for industry and individuals to tackle waste heat emissions, thus reducing expense in an environmentally respectful way. Yet, heat pipes are still little known to the general public and the widespread use of these devices has only been initiated. This is mainly due to high manufacturing costs that used to represent high investments for purchasers. However, with the recent progress made in the manufacturing processes of heat pipes, this technology is becoming more affordable and is attracting industrialists as the return-on-investment time of heat pipe-based installations is significantly reduced. For instance, in an economic assessment of the use of heat pipes in ventilation systems, Jouhara [16] reported a payback period of one month. Beyond the demonstrated potential of this technology, the complex mechanisms involved in heat pipes make them difficult to study from a theoretical point of view. Even if boiling has been widely studied in the last century, researchers still struggle to agree on the exact physical phenomena implied. A demonstration of this statement is the high number of heat transfer correlations reported in the literature. Up to today, it remains hazardous to predict boiling [17] and most of the boiling correlations reported have an accuracy of around $\pm 30 \%$ [18]. This represents the main obstacle in large scale heat pipe implementation as the difficulty in delivering accurate predictions of heat pipe performance leads to inaccurate payback time estimates for potential investors. In that regard, boiling mechanisms are still intensively studied today. However, to the authors' knowledge, the particular case of nucleate pool boiling applied to thermosyphons has been little reviewed so far. Moreover, the need to gather and analyse the high number of nucleate boiling heat transfer correlations can be felt. Indeed, for many researchers using heat pipes in industrial applications, it is difficult to select and use suitable boiling correlations. Hence, a deeper understanding of two-phase phenomena is to be provided. By reviewing the main boiling mechanisms occurring in gravity assisted two-phase closed thermosyphons (TPCTs) this paper intends to provide a basic knowledge of nucleate pool boiling heat transfer in wickless heat pipes. 


\section{Nomenclature}

A

Ar

$B_{o}$

C

$\mathrm{Ca}$

Co

$c_{p}$

$C_{s f}$

D

$D_{d}$

$f_{d}$

$g$

h

$h^{*}$

$i_{l v}$

$J$

Ja

$k$

$k_{\text {bolt }}$

$k_{f}$

$L$

$L_{b}$

$\dot{m}$

$M_{\text {mol }}$

$N_{a}$

$N_{\text {mol }}$

$\mathrm{Nu}$

$N_{0}$

$P$

$P_{a t m}$

$P_{\text {crit }}$

Pr
Surface area

Archimedes number, $\left(A r=g L^{3} \rho_{l}\left(\rho_{l}-\rho_{v}\right) / \mu^{2}\right)$

Bond number, $\left(B_{o}=g\left(\rho_{l}-\rho_{g}\right) D_{d}{ }^{2} / \sigma\right)$

Constant

Capillary number, $\left(\mathrm{Ca}=\mu_{v} U / \sigma \cos \theta\right)$

Confinement number, $\left(C o=L_{b} / D_{i}\right)$

Specific heat

Constant in Rohsenow correlation depending on the surface-fluid combination

Diameter

Bubble departure diameter

Bubble departure frequency

Gravitational acceleration

Heat transfer coefficient

Dimensionless heat transfer coefficient,

$\left(h^{*}=h / P_{\text {crit }}\left(R_{\text {mol }} / M_{\text {mol }} T_{\text {crit }}\right)^{1 / 2}\right)$

Latent heat of vaporization

Density of nuclei per unit of volume and time

Jakob number, $\left(J a=\Delta T c_{p, l} \rho_{l} / \rho_{v} i_{l v}\right)$

Thermal conductivity

Boltzmann constant, $1.38065 \times 10^{-23}$

Molecular evaporation rate

Length

Bubble length scale, $\left(L_{b}=\left[\sigma / g\left(\rho_{l}-\rho_{v}\right)\right]^{1 / 2}\right)$

Mass flow rate

Molecular weight

Number of active nucleation sites per unit surface area

Avogadro number, $\left(N_{m o l}=6.022 \times 10^{20}\right)$

Nusselt number, $(N u=h L / k)$

Number density of single activated molecules

Pressure

Atmospheric pressure

Critical pressure

Prandtl number, $\left(\operatorname{Pr}=\mu c_{p} / k\right)$ $\mathrm{m}^{2}$

dimensionless

dimensionless

dimensionless

dimensionless

dimensionless

$\mathrm{J} . \mathrm{kg}^{-1} \cdot \mathrm{K}^{-1}$

dimensionless

$\mathrm{m}$

m

$\mathrm{s}^{-1}$

$\mathrm{m} \cdot \mathrm{s}^{-2}$

$\mathrm{W} \cdot \mathrm{m}^{-2} \cdot \mathrm{K}^{-1}$

dimensionless

$$
\begin{gathered}
\mathrm{J} \cdot \mathrm{kg}^{-1} \\
\mathrm{~m}^{-3} \cdot \mathrm{s}^{-1}
\end{gathered}
$$

dimensionless

W. $\mathrm{m}^{-1} \cdot \mathrm{K}^{-1}$

$\mathrm{m}^{2} \cdot \mathrm{kg} \cdot \mathrm{s}^{-2} \cdot \mathrm{K}^{-1}$

molecules. $\mathrm{s}^{-1}$

m

m

kg. $\mathrm{s}^{-1}$

kg. $\mathrm{kmol}^{-1}$

$\mathrm{m}^{-2}$

$\mathrm{kmol}^{-1}$

dimensionless

$\mathrm{m}^{-2}$

N. $\mathrm{m}^{-2}$

N. $\mathrm{m}^{-2}$

N. $\mathrm{m}^{-2}$

dimensionless 


\begin{tabular}{|c|c|c|}
\hline$P^{*}$ & Dimensionless reduced pressure, $\left(P^{*}=P / P_{\text {crit }}\right)$ & N. $m^{-2}$ \\
\hline$q$ & Heat flow rate & $\mathrm{W}$ \\
\hline$q^{\prime \prime}$ & Heat flux per unit surface area & W. $\mathrm{m}^{-2}$ \\
\hline$q^{*}$ & $\begin{array}{l}\text { Dimensionless heat flux, } \\
\left(q^{*}=q^{\prime \prime} / P_{\text {crit }}\left(R_{\text {mol }} T_{\text {crit }} / M_{\text {mol }}\right)^{1 / 2}\right)\end{array}$ & dimensionless \\
\hline$r$ & Radius & $\mathrm{m}$ \\
\hline$r_{c a v}$ & Radius of a cavity & $\mathrm{m}$ \\
\hline$r_{c a v, \max }$ & Maximum radius of cavities on the surface & $\mathrm{m}$ \\
\hline$r_{s}$ & Radius of the largest cavity on the surface & $\mathrm{m}$ \\
\hline$r^{+}$ & Dimensionless radius in Eq. (25) and Eq. (29) & dimensionless \\
\hline$R$ & Thermal resistance & $\mathrm{K} . \mathrm{W}^{-1}$ \\
\hline$R_{m o l}$ & Molar specific gas constant, $\left(R_{m o l}=8314.4598\right)$ & $\mathrm{J} \cdot \mathrm{K}^{-1} \cdot \mathrm{kmol}^{-1}$ \\
\hline$R_{a}$ & $\begin{array}{l}\text { Arithmetic mean deviation of the profile } \\
\text { (Mittenräuwert), ISO4287-1:1984/DIN4762 }\end{array}$ & $\mathrm{m}$ \\
\hline$R_{a, p}$ & Average roughness parameter & $\mu \mathrm{m}$ \\
\hline$R e$ & Reynolds number & dimensionless \\
\hline$R_{p}$ & $\begin{array}{l}\text { Maximum peak height of the profile (Glättungstiefe), } \\
\text { ISO4287-1 : 1984/DIN4762 }\end{array}$ & $\mathrm{m}$ \\
\hline$R^{*}$ & $\begin{array}{l}\text { Dimensionless } \quad \text { surface } \quad \text { roughness } \\
\left(R^{*}=R_{a} /\left(R_{\text {mol }} T_{\text {crit }} / P_{\text {crit }} N_{\text {mol }}\right)^{1 / 3}\right)\end{array}$ & dimensionless \\
\hline$t$ & Time & s \\
\hline$t_{g}$ & Bubble growth period & $\mathrm{s}$ \\
\hline$t_{w}$ & Bubble waiting period & $\mathrm{s}$ \\
\hline$t^{+}$ & Dimensionless time in Eq. (25) and Eq. (29) & dimensionless \\
\hline$T$ & Temperature & $\mathrm{K}$ \\
\hline$T_{\text {crit }}$ & Critical temperature & $\mathrm{K}$ \\
\hline$T_{r}$ & Reduced temperature, $\left(T / T_{\text {crit }}\right)$ & dimensionless \\
\hline$v$ & Specific volume & $\mathrm{m}^{3} \cdot \mathrm{kg}^{-1}$ \\
\hline$v_{r}$ & Reduced specific volume, $\left(v / v_{\text {crit }}\right)$ & dimensionless \\
\hline$V$ & Volume & $\mathrm{m}^{3}$ \\
\hline$U$ & Velocity & $\mathrm{m} \cdot \mathrm{s}^{-1}$ \\
\hline \multicolumn{3}{|c|}{ Greek Symbols } \\
\hline$\alpha$ & Thermal diffusivity, $\left(\alpha=k / \rho c_{p}\right)$ & $\mathrm{m}^{2} \cdot \mathrm{s}^{-1}$ \\
\hline$\Gamma$ & Coefficient to consider the possibility of nuclei decay & dimensionless \\
\hline$\Delta P_{\text {sat }}$ & $\begin{array}{l}\text { Difference in saturation (vapour) pressure between the } \\
\text { wall temperature and the saturation temperature, } \\
\left(\Delta P_{s a t}=\left.P_{v}\right|_{T=T_{w}}-\left.P_{v}\right|_{T=T_{s a t}}\right)\end{array}$ & $\mathrm{Pa}$ \\
\hline
\end{tabular}


$\Delta P_{0} \quad$ Initial pressure difference between interior and exterior of the bubble

$\Delta R \quad$ Boiling space dimension

$\mathrm{m}$

$\Delta T_{\text {excess }} \quad$ Excess temperature between the hot surface and the saturated liquid, $\left(\Delta T_{\text {excess }}=\left(T_{s}-T_{\text {sat }}\right)\right)$

K

Wall superheat, difference of temperature between the

$\Delta T_{\text {sat }} \quad$ wall and the saturation temperature of the liquid, $\left(\Delta T_{\text {sat }}=\left(T_{w}-T_{\text {sat }}\right)\right)$

$\rho \quad$ Density

$\sigma \quad$ Surface tension

kg. $\mathrm{m}^{-3}$

$\theta \quad$ Contact angle

$\theta_{a} \quad$ Contact angle of an advancing wave

N. $\mathrm{m}^{-1}$

$\mu \quad$ Dynamic viscosity

$v \quad$ Kinematic viscosity, $(v=\mu / \rho)$

$\psi \quad$ Mixing Coefficient in El-Genk [19] correlation

$\psi_{m} \quad$ Mouth angle of a cavity

\section{Subscripts}

a $\quad$ Adiabatic

atm Atmospheric

$b \quad$ Bubble

boiling Boiling

c Condenser

cav Cavity

Condensation Condensation

crit Critical

$d \quad$ Departure

e Evaporator

excess Excess

ext External

$\mathrm{fb} \quad$ Falling film boiling

$g \quad$ Growth

$h p \quad$ Heat pipe

$i \quad$ Inner

in Internal

$l \quad$ Liquid

$\max \quad$ Maximum

mol Molar

$n b \quad$ Nucleate boiling 


$\begin{array}{cl}n c & \text { Natural convection } \\ o & \text { Out } \\ p b & \text { Pool boiling } \\ r & \text { Reduced } \\ s & \text { Solid } \\ s a t & \text { Saturation } \\ v & \text { Vapour } \\ W & \text { Wall } \\ w & \text { Waiting time } \\ x & \text { Axial } \\ 0 & \text { Standard conditions }\end{array}$

\section{Superscripts}

Per surface area

$$
\mathrm{m}^{-2}
$$

Dimensionless

dimensionless

Per unit of time

$$
\mathrm{s}^{-1}
$$

\section{Acronyms}

CFD Computational fluid dynamic

CHF Critical heat flux

CSF Continuum surface force model

FR Filling ratio

HTC Heat transfer coefficient

HVAC Heating, ventilation and air conditioning

RMS Root mean square (deviation)

TPCT Two-phase closed thermosyphon

UDF User defined function

VOF Volume of fluid

\section{BOILING IN TWO-PHASE THERMOSYPHONS}

Defined as the process in which the addition of heat to a liquid provokes the creation of vapour, boiling is a wide scientific area in which many different phenomena can occur depending on the geometry, operating conditions, heating method, fluid flow, etc... Despite the fact that both evaporation and boiling are liquid-to-vapour phase change processes, it seems important to distinguish between them. On the one hand, evaporation takes place at the liquid-vapour interface and occurs when the vapour pressure is less than the saturation pressure of the liquid. On the other hand, boiling takes place at the solid-liquid interface. In that case, the liquid is in contact with a hot surface, the temperature of which is above the saturation temperature, which forces the liquid to turn to vapour and create bubbles [20]. Boiling can be subdivided into the following categories [21]:

- Pool boiling: 
In pool boiling, no motion of the liquid by external means is implied. The only motion of fluid within the liquid pool is created by natural convection currents and bubble formation. A typical example of pool boiling is water boiling in a pan.

- Falling film boiling and thin-film evaporation:

In thin film evaporation and falling film boiling, a liquid flows by gravity on a heated surface. At low heat flux, the thin film only absorbs heat and evaporation can be observed at the liquid surface. Yet, at higher heat fluxes, bubbles can appear at the solid-liquid interface and falling film boiling occurs.

- $\quad$ Forced convective boiling (often defined as Flow Boiling):

In forced convective boiling, the liquid is forced to flow inside a heated passage by the action of external means. The vapour is trapped inside the section and flows in the same direction as the liquid phase, thus forming a two-phase flow. Interactions between the liquid and vapour phases are strong. The most common example of forced convective boiling is water circulating through a heated pipe.

\section{- Cross flow boiling:}

In cross flow boiling, the liquid is forced to flow across a heated surface. A typical example of cross flow boiling is water flowing through a bank of heated cylinders in which the direction of the flow is normal to the cylinders' axes.

\section{- Hot surface rewetting}

As its name indicates, in hot surface rewetting a fluid comes into contact with a hot dry surface. The surface is rewetted and a violent generation of vapour can be observed. This type of boiling can be illustrated by the quenching of a hot metal.

In thermosyphons, the two-types of boiling mainly considered are pool boiling and thin film evaporation/falling film boiling. As briefly introduced, thermosyphons use a phase change cycle of a working fluid to transfer large amounts of heat. Indeed, boiling heat transfer can reach higher heat transfer coefficients (HTCs) in comparison to conventional conduction, convection or radiative heat transfer. This can be explained as the temperature of the fluid is almost constant during a phase change. The energy received is transported under the form of latent heat. As a form of convection heat transfer from a surface to a liquid, boiling is governed by Newton's law of cooling:

$$
q_{\text {boiling }}=h\left(T_{s}-T_{\text {sat }}\right)=h \Delta T_{\text {sat }}
$$

where $q_{\text {boiling }}$ is the boiling heat flux per surface unit area $\left(\mathrm{W} \cdot \mathrm{m}^{-2}\right), h$ is the heat transfer coefficient $\left(\mathrm{W} . \mathrm{m}^{-2} \cdot \mathrm{K}^{-1}\right)$ and $\left(T_{s}-T_{s a t}\right)$ is the temperature difference between the heated solid surface and the saturated liquid (K). In thermosyphons, pool boiling takes place at the evaporator. While receiving heat from a heat source, the temperature of the evaporator wall increases above the saturation point of the liquid which causes the onset of the phase change of the working fluid in the pool. The energy given by the hot wall is carried by the vapour as latent heat transport:

$$
q=\dot{m} i_{l v}
$$

where $q$ is the heat flux (W), $\dot{m}$ is the mass flow rate $\left(\mathrm{kg} . \mathrm{s}^{-1}\right)$ and $i_{l v}$ is the latent heat of vaporization $\left(\mathrm{J} . \mathrm{kg}^{-1}\right)$. Attracted by the temperature gradient inside the heat pipe, the vapour flows to the cold section of the thermosyphon, the condenser. Due to the heat sink contact, the wall temperature of the condenser is below the saturation point of the working fluid. This forces the vapour to condense on the cold wall, thus releasing its latent heat energy. Finally, the condensate returns to the thermosyphon evaporator by forming a thin liquid film on the wall. This operational cycle of a thermosyphon in addition to the detailed view of pool boiling and falling film boiling is presented in Fig. 1. 


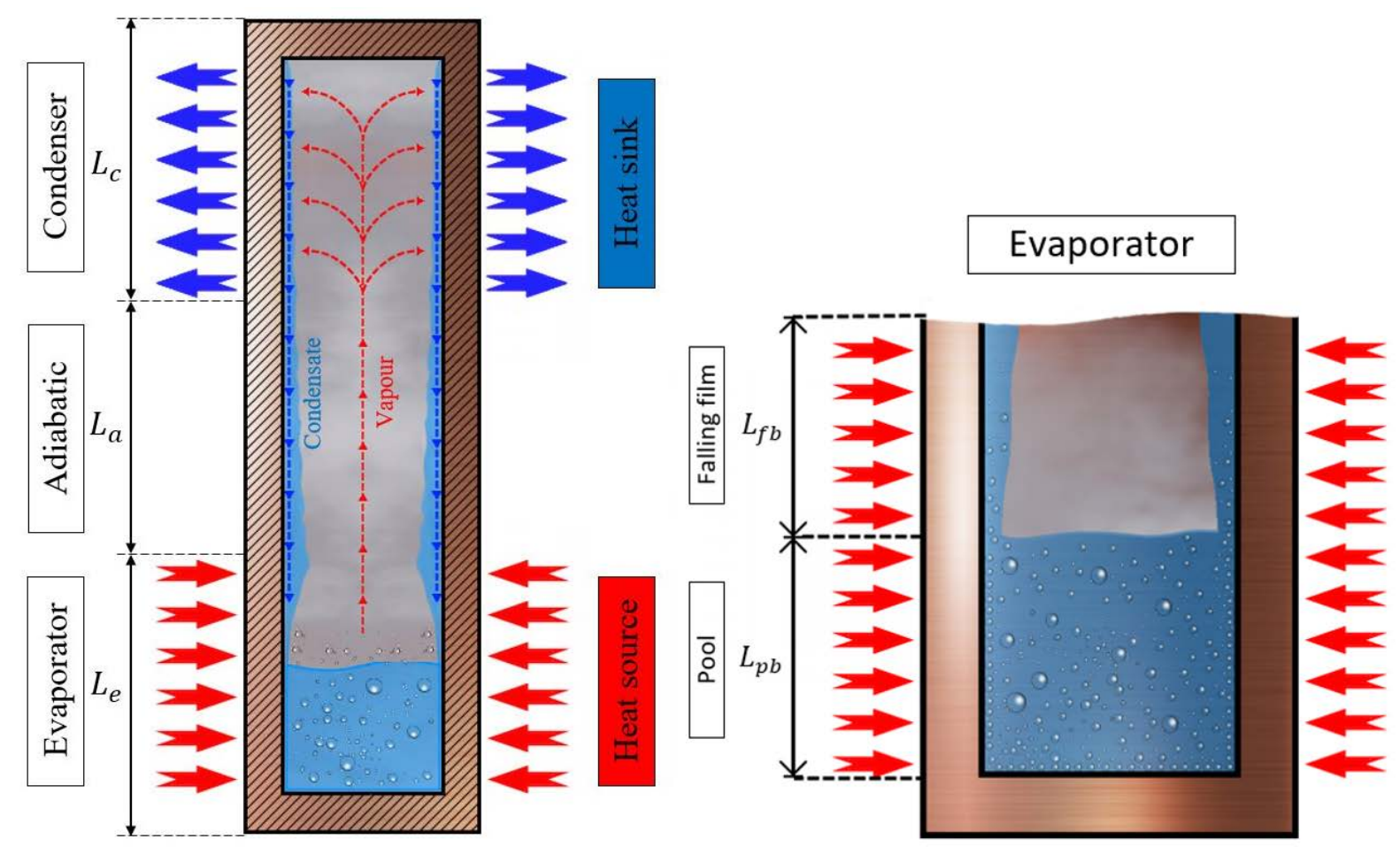

Figure 1: Working cycle of a thermosyphon and boiling mechanisms at the evaporator, case where both falling film and pool boiling take place with a filling ratio $\mathrm{FR} \approx 50 \%$

In Fig. 1, the filling ratio $F R$ of the thermosyphon presented, which represents the ratio of the liquid pool volume to the volume of the evaporator, is lower than $100 \%$. Indeed, in the case where $F R<$ $100 \%$, both falling film boiling and pool boiling take place at the evaporator. However, in the case where the pool completely fills the evaporator $(F R=100 \%)$, pool boiling only occur.

To estimate the performance of a heat pipe, the thermal resistance similarity is used. The electro-thermal analogy considered is presented in Fig. 2. 

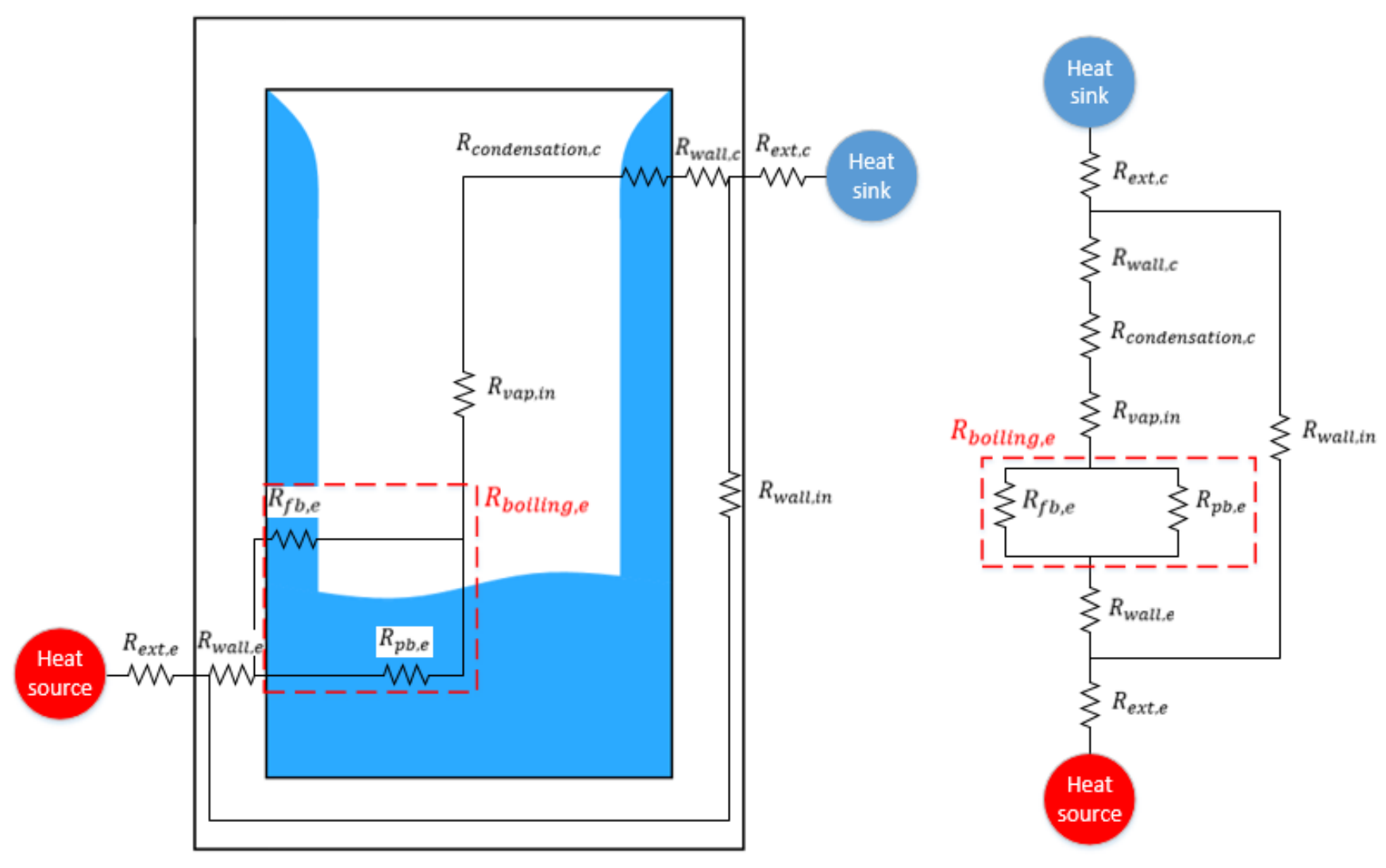

Figure 2: Thermal resistance analogy of a two-phase thermosyphon

The total heat transfer through the pipe can be obtained from:

$$
q_{t o t}=\frac{\Delta T}{R_{h p}}
$$

where $\Delta T$ is the temperature difference between the heat source and heat sink $(\mathrm{K})$ and $R_{h p}$ is the total

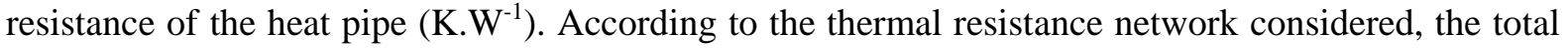
resistance of the heat pipe $R_{h p}$ can be estimated by:

$$
\begin{aligned}
R_{h p}=R_{\text {ext }, e}+ & R_{\text {ext }, c} \\
& +\left[\frac{1}{R_{\text {wall }, \text { in }}}\right. \\
& \left.+\frac{1}{R_{\text {wall }, e}+R_{\text {boiling }, e}+R_{\text {vap }, \text { in }}+R_{\text {condensation }, c}+R_{\text {wall }, c}}\right]
\end{aligned}
$$

where $R_{\text {ext,e }}$ and $R_{\text {ext,c }}$ are the external resistances at the evaporator and condenser, respectively, $R_{\text {wall,in }}$ is the axial conduction resistance of the wall, $R_{\text {wall,e }}$ and $R_{\text {wall,c }}$ are the radial conduction resistance of the wall at the evaporator and condenser, $R_{\text {boiling,e }}$ is the boiling resistance at the evaporator, $R_{\text {vap,in }}$ is the internal vapor resistance due to pressure drop and $R_{\text {condensation, }}$ is the condensation resistance at the condenser. While the boiling resistance is detailed hereafter, the values of the other resistances are briefly expressed in Table 1 [21], [22]. The subscripts e, a and c refer to evaporator, adiabatic and condenser, respectively. 
Table 1: Thermal resistances of a thermosyphon

\begin{tabular}{|c|c|}
\hline Resistance & Nomenclature \\
\hline $\begin{aligned} R_{\text {ext }, e} & =1 /\left(h_{o, e} A_{o, e}\right) \\
R_{\text {ext }, c} & =1 /\left(h_{o, c} A_{o, c}\right)\end{aligned}$ & $\begin{array}{l}h_{o} \text { is the external heat transfer } \\
\text { coefficient } \\
* \quad A_{o} \text { is the external area in contact with the } \\
\text { heat source/heat sink }\end{array}$ \\
\hline$R_{\text {wall,in }}=\left(0.5 L_{e}+L_{a}+0.5 L_{c}\right) /\left(A_{x} k_{w}\right)$ & $\begin{array}{l}A_{x} \text { is the cross-sectional area of the wall } \\
L \text { is the length } \\
k_{w} \text { is the wall thermal conductivity }\end{array}$ \\
\hline $\begin{array}{l}R_{\text {wall }, e}=\ln \left(D_{o} / D\right) /\left(2 \pi L_{e} k_{w}\right) \\
R_{\text {wall }, c}=\ln \left(D_{o} / D\right) /\left(2 \pi L_{c} k_{w}\right)\end{array}$ & $\begin{array}{l}\text { * } D_{o} \text { is the external diameter of the } \\
\text { thermosyphon } \\
* \quad D \text { is the internal diameter of the } \\
\text { thermosyphon }\end{array}$ \\
\hline$R_{v a p, \text { in }}=T_{v}\left(P_{v, e}-P_{v, c}\right) / \rho_{v} i_{l v} q$ & $\begin{array}{l}T_{v} \text { is the temperature of the vapour } \\
P_{v} \text { is the vapour pressure } \\
q \text { is the heat flow rate }\end{array}$ \\
\hline$R_{\text {condensation }, c}=1 /\left(h_{\text {condensation }} A_{c, \text { in }}\right)$ & $\begin{array}{l}* h_{\text {condensation }} \text { is the condensation heat } \\
\text { transfer coefficient } \\
* \quad A_{c, \text { in }} \text { is the inside area of the condenser }\end{array}$ \\
\hline
\end{tabular}

With regard to the equivalent boiling resistance presented in Fig. 2, the boiling resistance at the evaporator can be obtained by:

- In the case where both pool boiling and falling film boiling are considered (FR $<100 \%)$

$$
\frac{1}{R_{\text {boiling,e }}}=\frac{1}{R_{p b, e}}+\frac{1}{R_{f b, e}}=\frac{L_{p b, e}+L_{f b, e}}{L_{p b, e} R_{p b, e}}+\frac{L_{p b, e}+L_{f b, e}}{L_{f b, e} R_{f b, e}}
$$

which can be reformulated as:

$$
R_{\text {boiling,e }}=\frac{1}{h_{p b, e} A_{p b, e}+h_{f b, e} A_{f b, e}}=\frac{1}{h_{p b, e} A_{p b, e}+h_{f b, e}\left(A_{e}-A_{p b, e}\right)}
$$

The importance of each boiling regime between pool boiling and falling film boiling is related to the filling ratio. Indeed, for small filling ratios, the height of the pool is decreased while the length of the film in contact with the heated section is increased. Therefore, in that case, falling film boiling becomes more important relative to pool boiling. The boiling resistance of each regime can be related to the filling ratio $F R$ by expressing the surface areas as:

$$
A_{p b, e}=\pi D_{i n} L_{e} F R \text { and } A_{f b, e}=\pi D_{i n} L_{e}(1-F R)
$$

- In the case where only pool boiling is considered ( $\mathrm{FR}=100 \%)$

$$
R_{\text {boiling,e }}=R_{p b, e}=\frac{1}{h_{p b, e} A_{p b}}
$$

In this study, the special case where only pool boiling is considered, i.e. for thermosyphons with a filling ratio $\mathrm{FR}=100 \%$, is investigated. To predict the performance of a thermosyphon in the case where only pool boiling is considered, it can be deduced from the preceding equation that the pool boiling heat transfer coefficient is required. The development of correlations to estimate the heat transfer coefficient in pool boiling is a challenge researchers are still facing. Indeed, the complexity of the mechanisms involved, the difficulties of observing and explaining the micro phenomena, the lack of reproducibility between the experiments and the important discrepancy between the authors' observations make the development of predicting correlations hazardous. For heat pipes users, estimating the boiling resistance 
of an installation remains tough regarding the high number of correlations reported in the literature and the complexity of the two-phase boiling heat transfer occurring. The objective of the following sections is to review and clarify the reported scientific phenomena taking place in pool boiling for the special application in thermosyphons. In order to ease the selection of suitable boiling correlations in the prediction of thermosyphon performance, numerous correlations are reported and recommendations on their use are made.

\section{BUBBLES AND NUCLEATION}

In pool boiling, the highest heat transfer coefficients are obtained for nucleate boiling regimes. In nucleate boiling, the high heat flux imposed on the heating surface forces the liquid phase to turn to vapour, thus creating a bubble. Bubbles represent the centre point of boiling, as their presence, population density, diameter, shape, release frequency and characteristics rule boiling heat transfer. Despite the high number of studies on bubbles, the mechanisms by which the bubble action determines the heat transfer coefficient is still not clearly understood [23]. Yet, explaining the scientific phenomena related to bubbles is a key to determining accurate correlations of boiling heat transfer.

\subsection{Critical radius and equilibrium conditions}

In this section, the case of a bubble under equilibrium conditions and surrounded by liquid is considered. The bubble owes its existence to the surface tension $\sigma$ at the liquid-vapor interface. The difference of temperature between the vapour bubble and the bulk liquid at its contact define the intensity of the surface tension force. At the critical temperature of the fluid, the surface tension becomes zero. This explains why no bubbles are formed at supercritical pressure and temperature. To define the equilibrium conditions allowing the existence of a bubble, an energy balance on a control volume between the bubble and the surrounding liquid leads to [21]:

$$
\sigma d A_{i}-P_{v} d V_{v}-P_{l} d V_{l}=0
$$

where $\sigma$ is the surface tension $\left(\mathrm{J} \cdot \mathrm{m}^{-2}\right), A_{i}$ is the sphere surface of the bubble $\left(\mathrm{m}^{2}\right), P_{v}$ and $P_{l}$ are the vapor and liquid pressure $\left(\mathrm{J}^{-3}{ }^{-3}\right.$ ) and $V_{v}$ and $V_{l}$ are the vapor and liquid volumes $\left(\mathrm{m}^{3}\right)$. For a system with constant temperature and volume $\left(d V_{v}=-d V_{l}\right)$, the Young-Laplace equation relating the difference between the vapour pressure and liquid pressure to the bubble surface tension can be deduced:

$$
P_{v}-P_{l}=\sigma \frac{d A_{i}}{d V_{v}}=\frac{2 \sigma}{r}
$$

where $r$ is the bubble radius. By integrating this pressure difference expressed with the ClapeyronClausius equation, in the case where the kinematic viscosity ratio between the liquid and the vapour phase is such that $v_{l} / v_{v} \ll 1$, the critical radius of a bubble $r_{c r i t}$ to reach equilibrium conditions is given as [21]:

$$
r_{c r i t}=2 \sigma T_{s a t} v_{v} /\left(i_{l v} \Delta T_{s a t}\right)
$$

where $T_{s a t}$ is the saturation temperature of the fluid (K), $v_{v}$ is the kinematic viscosity of the vapour $\left(\mathrm{m}^{2} . \mathrm{s}^{-1}\right), i_{l v}$ is the latent heat of vaporization $\left(\mathrm{J} \mathrm{kg}^{-1}\right)$ and $\Delta T_{s a t}$ is the difference between the temperature of the system and the saturation temperature. For circular bubbles surrounded by liquid fluid without any external influences, bubbles with a radius lower than the critical radius will collapse under the influence of the liquid pressure. On the contrary, bubbles with higher radius will grow due to a higher surface tension.

\subsection{Nucleation process}

The process during which a bubble is created is called nucleation. The nucleation is of importance in the prediction of the density of active nucleation sites during boiling. The more active nucleation sites are, the more bubbles will be formed and thus, the higher the heat transfer will be. It is commonly agreed that two types of nucleation processes exist regarding their location. In the case where the bubbles appear amongst the bulk of liquid, the nucleation is called "homogeneous nucleation". In the case where the bubble is formed near a surface at the solid-liquid interface, the process is designated "heterogeneous nucleation". In most of the boiling experiments, the prevailing nucleation process is 
heterogeneous nucleation due to lower energy needs in the creation of a bubble at the contact of a surface in comparison with a nucleation in the fluid. Homogeneous nucleation is rare.

In homogeneous nucleation, local fluctuations within the liquid bulk can form bubbles with a radius sufficiently important for them to persist. The amount of energy required for this process is high and the temperature of the liquid needs to be significantly superior to the saturation point of the working fluid. To estimate the temperature necessary to initiate a homogeneous process two limits can be considered: the thermodynamic limit and the kinetic limit. The thermodynamic limit can be explained using a Pressure-Volume (PV) phase diagram of the fluid that defines the regions of existence for both liquid and vapour phases. A PV phase diagram of water is presented in Fig. 3 [24].

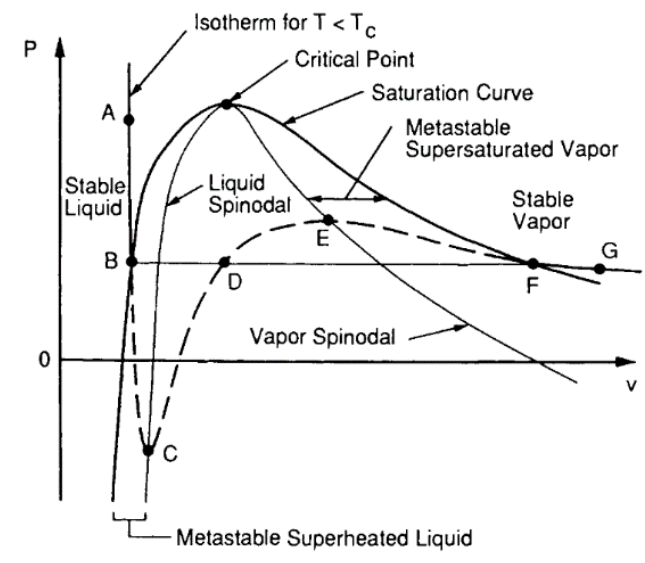

Figure 3: Pressure-volume phase diagram of water, from Carey [24]

It is stipulated that under some conditions of temperature, pressure and volume, the fluid transits from liquid phase to vapour phase when it reaches the liquid spinodal. The liquid spinodal, defined as the ultimate region beyond which the liquid turns to vapour (Point $\mathrm{C}$ ), is described by the condition:

$$
\left.\frac{\partial P}{\partial V}\right|_{T}=0
$$

From this analysis, Carey [24] estimated the temperature at which the liquid phase can no longer persist and gives the following reduced temperature $T_{r}$ to onset homogeneous nucleation as:

$$
T_{r}=\left[\frac{\left(3 v_{r}-1\right)^{2}}{4 v_{r}{ }^{3}}\right]^{1 /(\lambda+1)}
$$

where $T_{r}=T / T_{\text {crit }}$ is the reduced temperature, $v_{r}=v / v_{\text {crit }}$ is the reduced specific volume and $\lambda$ is a coefficient. This correlation can easily be compared to Van der Waals equation $(\lambda=0)$ and Berthelot equation $(\lambda=1)$ by adjusting the value of the parameter $\lambda$. By correcting a first empirical correlation by Lienhard [25], Lienhard et al.[26] proposed the following reduced temperature for the liquid spinodal of a wide range of fluids:

$$
T_{r}-T_{r, s a t}=0.923-T_{r, s a t}+0.077 T_{r, s a t}{ }^{9}
$$

Yet, this approach can be discussed, as the phase diagram and in particular the liquid spinodal is defined for systems with very limited disturbances, imperfections, and with little molecular interactions and collisions, which is rarely the case for real fluids as discussed by Lienhard [26]. Therefore, the analogy between liquid spinodal and homogeneous nucleation must be used with caution. The kinetic limit principle stipulates that microscopic seeds of vapor, called "nuclei", reside randomly in the liquid. To form a nucleus with a diameter greater than the critical diameter, it was found that the density of nuclei per unit of volume and time $J$ must be high (typically $J>10^{12}$ [21]). Avedisan [27] reported the following expression for the mean rate of nuclei formation $J$ :

$$
J=\Gamma k_{f} N_{0} \exp \left\{\frac{-16 \pi \sigma^{3}}{3 k_{\text {bolt }} T_{l}\left[\eta P_{\text {sat }}\left(T_{l}\right)-P_{l}\right]^{2}}\right\}
$$


where $\Gamma$ is a factor to consider the possibility that a nucleus can decay ( $\Gamma$ is close to 1 ), $k_{f}$ is the molecular evaporation rate (molecule. ${ }^{-1}$ ), $N_{0}$ is the number density of single activated molecules, $\sigma$ is the surface tension $\left(\mathrm{N} . \mathrm{m}^{-1}\right), \quad k_{\text {bolt }}$ is the Boltzmann constant $\left(k_{\text {bolt }}=1.38065 \times\right.$ $\left.10^{-23} \mathrm{~m}^{2} \cdot \mathrm{kg} \cdot \mathrm{s}^{-2} \cdot \mathrm{K}^{-1}\right), P_{s a t}\left(T_{l}\right)$ is the saturation pressure of the liquid at temperature $T_{l}$ and $\eta$ is given by:

$$
\eta=\exp \left\{\frac{P_{l}-P_{s a t}\left(T_{l}\right)}{\rho_{l} R_{m o l} T_{l}}\right\}
$$

where $R_{m o l}$ is the molar specific gas constant $\left(R_{m o l}=8314.4598 \mathrm{~J} \cdot \mathrm{K}^{-1} \cdot \mathrm{kmol}^{-1}\right)$. According to the work by Avedisan [27], the factor $\Gamma k_{f}$ can be approximated by:

$$
\Gamma k_{f} \approx \frac{2 \sigma}{M_{m o l}}
$$

where $M_{m o l}$ is the molecular weight $\left(\mathrm{kg} \cdot \mathrm{kmol}^{-1}\right)$. Carey [24] proposed a similar, yet simpler form of the mean rate of nuclei formation $J$ :

$$
J=1.44 \times 10^{40}\left(\frac{\rho_{l}^{2}}{M_{\text {mol }}}\right)^{1 / 2} \exp \left\{\frac{-16 \pi \sigma^{3}}{3 k_{\text {bolt }} T_{l}\left[\eta P_{\text {sat }}\left(T_{l}\right)-P_{l}\right]^{2}}\right\}
$$

with $\eta$ given by Eq. (15). By taking the criterion $J>10^{12}$, the temperature required by the kinetic condition to initiate homogeneous nucleation can be determined. Both thermodynamic and kinetic limits must be considered to anticipate the occurrence of homogeneous nucleation in a liquid. Yet, the temperature required in homogeneous nucleation is higher than those in heterogeneous nucleation, which is predominant. Typically, the superheat required to initiate homogeneous nucleation in a liquid bulk of water at atmospheric pressure is of the order of $200^{\circ} \mathrm{C}$ above the saturation temperature of the fluid. In comparison, the superheat needed is about $10-15^{\circ} \mathrm{C}$ for heterogeneous nucleation which fits the experimentally observed appearance of the first bubbles [21].

In heterogeneous nucleation, the nuclei initiating the formation of bubbles are situated near a hot surface and the nucleation process is eased by the presence of cavities on the surface. Indeed, for all types of surfaces including "smooth surfaces", microscopic cavities exist at the material surface and can generate a bubble. Moreover, the formation of a hotter layer of fluid close to the wall, designated as a "thermal layer", permit the bulk liquid to be locally at a higher temperature, which is favourable for bubble formation. This type of nucleation significantly predominates in boiling experiments and in particular in the boiling regimes occurring in thermosyphons. The strong impact of the surface on the nucleation process and thus on the boiling mechanisms is one of the main reasons of the difficulties encountered in an accurate prediction of boiling. To consider the influence of the surface on the bubble formation, the central notions of active nucleation sites and contact angle must be introduced. An active nucleation site consists of a cavity that actively generates a bubble. By its shape, a vapour nucleus is trapped in the micro cavity and grows under the contact with the hot surface. Even at the bubble departure, the cavity will remain filled by a small portion of vapour that will initiate the nucleation and the growth of the next bubble. It must be known that some cavities situated on the surface may not be active as they are filled with water. Therefore, the boiling heat transfer can be similar between two surfaces with a different number of cavities as the actual number of active cavities generating bubbles is similar. Typical diameters of active cavities $D_{\text {cav }}$ are in the range $2 \mu \mathrm{m}<D_{\text {cav }}<20 \mu \mathrm{m}$. The wettability of a cavity is related to the notion of contact angle. The contact angle between a droplet and a surface represents the angle at the base of the droplet on the surface. It is an intrinsic characteristic of wettability that small contact angles witness a high wettability of the surface and vice versa for high contact angles. This is illustrated in Fig. 4. 


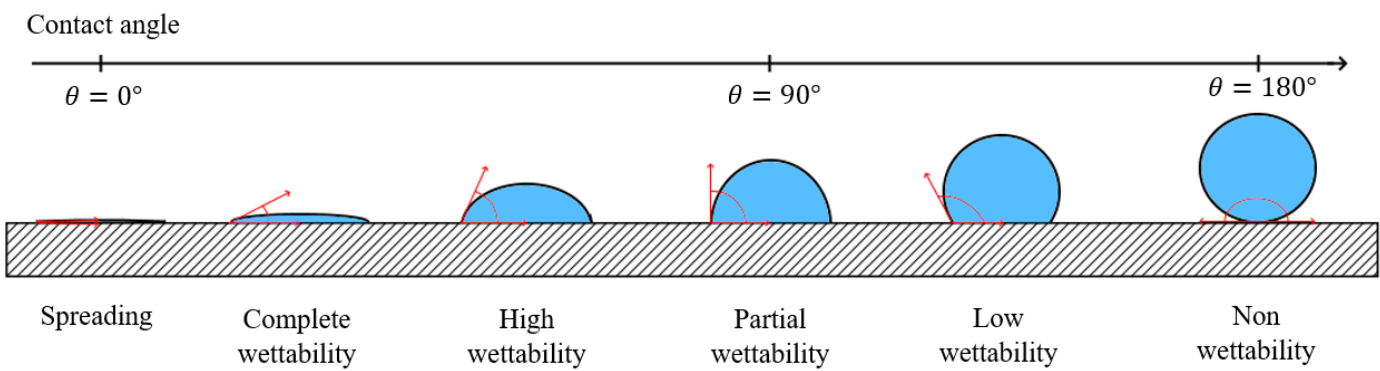

Figure 4: Contact angle of a droplet

In comparison to homogeneous nucleation, for a bubble growing on a planar surface (not in a cavity), Cole [28] and Rohsenow [29] attest that the energy required to generate a bubble is reduced by a factor $f(\theta)$ where $\theta$ is the contact angle given by:

$$
f(\theta)=\left[\frac{2+3 \cos \theta-\cos ^{3} \theta}{4}\right]^{1 / 2}
$$

Nevertheless, this energy is still much higher than the experimental observations of the first bubble appearance. To explain the lower energies required to initiate heterogeneous nucleation, the presence of cavities must be considered. The entrapment of small portions of gas in the micro cavities situated on the surface is mainly responsible for the easy formation of bubbles from a hot surface. Actually, the creation of the bubble is already initiated by the surface as a small amount of vapour is trapped in the existing cavity. Then, to develop a bubble, the energy provided is only necessary to permit the growth of the bubble seed. A model of heterogeneous nucleation was proposed by Hewitt [30], in which bubble growth from a cavity is schematized in Fig. 5.
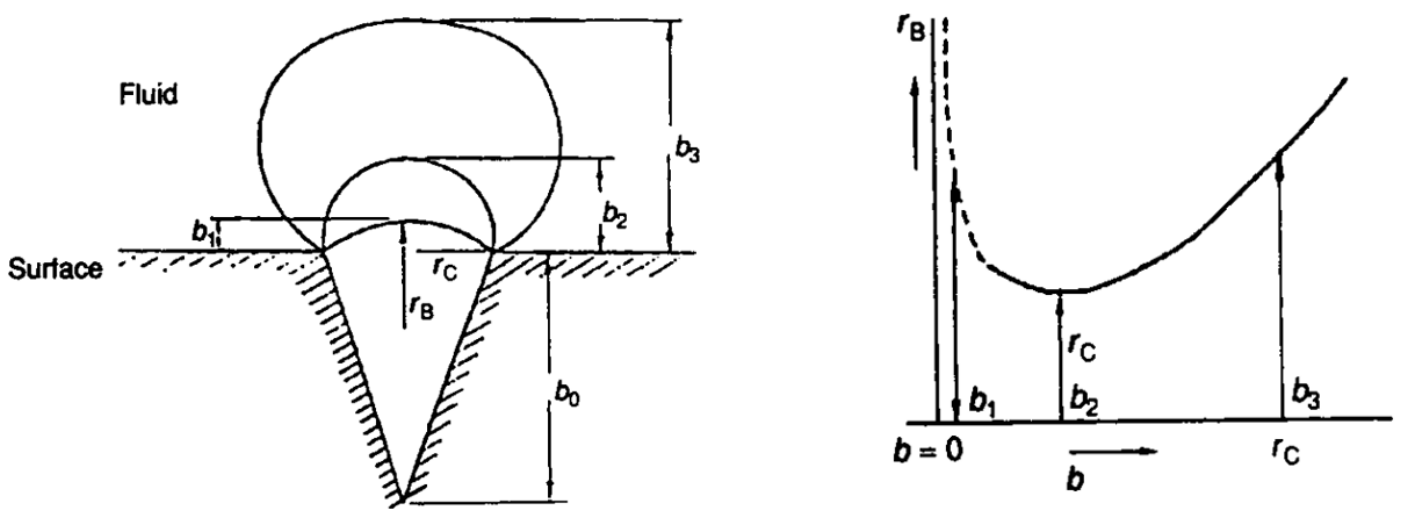

Figure 5: Hewitt [30] model of a heterogeneous nucleation from an idealized cavity

According to this model, the bubble radius is passing by an extremum value $r_{c}$. For a bubble to persist, this radius value must be greater than the critical radius defined in Eq. (11). Hence, using the critical radius expression of a bubble, the wall superheat required to initiate heterogeneous nucleation can be deduced [21]:

$$
\left(\Delta T_{\text {sat }}\right)_{W, \text { crit }}=T_{W, \text { crit }}-T_{\text {sat }}=\left(\frac{8 \sigma T_{s a t} v_{v} q^{\prime \prime}}{i_{l v} k_{l}}\right)^{1 / 2}
$$


where $T_{w, c r i t}$ is the wall critical temperature required to onset heterogeneous nucleation (K), $T_{s a t}$ is the saturation temperature of the fluid $(\mathrm{K}), v_{v}$ is the kinematic viscosity of the vapor $\left(\mathrm{m}^{2} \cdot \mathrm{s}^{-1}\right), q^{\prime}$ is the heat flux per surface area $\left(\mathrm{W} . \mathrm{m}^{-2}\right), i_{l v}$ is the latent heat of vaporization $\left(\mathrm{J} \mathrm{kg}^{-1}\right)$ and $k_{l}$ is the liquid thermal conductivity $\left(\mathrm{W} \cdot \mathrm{m}^{-1} . \mathrm{K}^{-1}\right)$. It has to be known that some inaccuracies can be observed in particular cases and that corrections of this expression are proposed in the literature. In parallel with the minimum wall superheat required to initiate a nucleation process from a cavity, another condition for the occurrence of heterogeneous nucleation is the entrapment of a small amount of gas in the cavity. Indeed, if a cavity is rewetted by the liquid, the nucleation site becomes inactive and bubbles are no longer formed. The condition of vapour entrapment in a cavity depends on the cavity geometry and on the contact angle of an advancing wave of liquid. The cavity model from Wang and Dhir [31] presented in Fig. 6 can be taken as a reference.

Figure 6: Cavity model from Wang and Dhir [31]

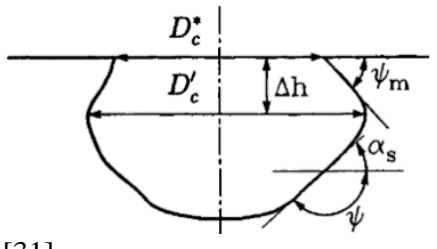

Consider that a wave of liquid is flowing over a surface, the condition for the entrapment of gas in the cavity is given by the Bankoff [32] criterion, relating the contact angle of the advancing liquid wave $\theta_{a}$ to the cavity mouth angle $\psi_{m}$ by:

$$
\theta_{a}>\psi_{m}
$$

If this criterion is verified, a small amount of vapour will be trapped in the micro cavity as the fluid flows over the surface. Thus, an active nucleation site will be formed when submitted to a high wall superheat.

As previously discussed, it has been shown that boiling is mostly governed by the formation of bubbles on the heated surface. The next difficulty in the prediction of boiling heat transfer is the estimation of the number of active nucleation sites $N_{a}$ that generate bubbles. Yet, the prediction of the number of active nucleation sites $N_{a}$ directly leads to a nucleate boiling heat transfer correlation. The large amount of work reported in the literature to measure and evaluate the density of active nucleation sites on a given surface witnesses the difficulty of this study as well as its importance. The number of active nucleation sites can be estimated using the Zhokhov [33]correlation:

$$
\sqrt{N_{a}}=25 \times 10^{-8}\left(\frac{i_{l v} \rho_{v} \Delta T}{T_{s a t} \sigma}\right)^{1.5}
$$

As observed in the work by Wang and Dhir [31], the number of active nucleation sites with a diameter greater than a given value $r_{\text {cav }}$ decreases with increasing radius $r_{\text {cav }}$. In other words, the density of active nucleation sites with small diameter is higher than the density of nucleation sites with greater diameters. According to the authors' experiments, for water on a copper surface with a contact angle $\theta=90^{\circ}$, the density of active nucleation sites with a diameter greater than $2 \mu \mathrm{m}$ was about $10^{4} \mathrm{sites} / \mathrm{cm}^{2}$ while it decreases to about $1 \mathrm{site} / \mathrm{cm}^{2}$ for nucleation sites with diameter greater than $10 \mu \mathrm{m}$. In accordance with this consideration, Mikic and Rohsenow [34] proposed to relate the number of active nucleation sites to the radius $r_{\text {cav }}$ of the cavity. The density of active nucleation sites with a radius greater than $r_{\text {cav }}$ is given by:

$$
N_{a}=C\left(\frac{r_{c a v, \text { max }}}{r_{\text {cav }}}\right)^{m}=C r_{\text {cav }, \text { max }}{ }^{m}\left(\frac{i_{l v} \rho_{v}}{2 T_{\text {sat }} \sigma}\right)^{m} \Delta T_{\text {sat }}^{m}
$$

where $C$ is an experiment-based constant often taken as $C=1$ unit. $m^{-2}, r_{\text {cav,max }}$ is the maximal active cavity radius on the surface $(\mathrm{m})$ and $m$ is a constant (typically, $=6.5$ ). Other correlations to estimate the number of active nucleation sites can be found in the recent review by Mohanty [35]. The number of active nucleation sites has a significant influence on boiling heat transfer in nucleate boiling. 
Therefore, the higher the number of nucleation sites at the evaporator, the better the performance of the thermosyphon.

\subsection{Bubble growth}

Similar to the homogeneous and heterogeneous nucleation that respectively take place in the liquid bulk and near a surface, two different mechanisms of bubble growth are to be considered. In the first case, after a homogeneous nucleation, the bubble grows within the liquid bulk. In the second case, following a heterogeneous nucleation process, the bubble grows from a surface. The objective is to describe the bubble radius evolution with time.

In the case where the bubble has been formed within the liquid bulk, two limits rule the bubble growth, designated as inertia-controlled growth and heat-transfer-controlled growth. In inertiacontrolled growth, the force exercised by the bubble pressing against the surrounding liquid determines the speed of growth of the bubble. Inertia-controlled growth usually takes place at the beginning of the growth. To describe the evolution of the bubble radius $r(t)$ with time $t$ in an inertia-controlled growth of a bubble, the Rayleigh [36] expression is taken as a reference:

$$
r(t)=\left\{\frac{2}{3}\left[\frac{T_{l}-T_{\text {sat }}\left(P_{l}\right)}{T_{\text {sat }}\left(P_{l}\right)}\right] \frac{\rho_{v}}{\rho_{l}} i_{l v}\right\}^{1 / 2} t
$$

In heat-transfer controlled growth, the limit governing the process is the capacity of the liquid to vaporize at the bubble-liquid interface. Heat-transfer-controlled growth takes place after inertiacontrolled growth, in the later stages of bubble formation within the bulk fluid. The evolution of the bubble radius $r(t)$ with time $t$ in heat-transfer-controlled growth is given by the equation by Plesset and Zwick [37]:

$$
r(t)=\frac{2 \Delta T_{s a t} k_{l}}{i_{l v} \rho_{v}}\left(\frac{3 t}{\pi \alpha_{l}}\right)^{1 / 2}
$$

where $k_{l}$ is the thermal conductivity of the liquid $\left(\mathrm{W} \cdot \mathrm{m}^{-1} \cdot \mathrm{K}^{-1}\right)$ and $\alpha_{l}$ is the liquid thermal diffusivity $\left(\mathrm{m}^{2} \cdot \mathrm{s}^{-1}\right)$. Yet, for a bubble growth within the liquid bulk, it is convenient to use correlations that consider both inertia-controlled growth for the early stages and heat-transfer-controlled growth for the later stages. One of the first widely used equations to describe the entire range of a bubble growth from the bulk superheated liquid is the correlation by Mikic et al. [38]:

where,

$$
r^{+}=\frac{2}{3}\left[\left(t^{+}+1\right)^{3 / 2}-\left(t^{+}\right)^{3 / 2}-1\right]
$$

$$
\begin{gathered}
r^{+}=\frac{r(t) A}{B^{2}} \text { and } t^{+}=\frac{t A^{2}}{B^{2}} \\
A=\left\{\frac{2\left[T_{l}-T_{s a t}\left(P_{l}\right)\right] i_{l v} \rho_{v}}{\rho_{l} T_{s a t}\left(P_{l}\right)}\right\}^{1 / 2} \\
B=\left(\frac{12 \alpha_{l}}{\pi}\right)^{1 / 2} J a=\left(\frac{12 \alpha_{l}}{\pi}\right)^{1 / 2}\left\{\frac{\left[T_{l}-T_{s a t}\left(P_{l}\right)\right] c_{p, l} \rho_{l}}{i_{l v} \rho_{v}}\right\}
\end{gathered}
$$

where $J a$ is the Jakob number. However, by assuming that the linearized Clausius-Clapeyron equation can be used to relate vapour pressure and temperature, by considering a constant vapour density and by neglecting the influence of the surface tension on the internal pressure of the bubble, this correlation has shown inaccuracies, especially during the initial stages at high superheats [21], [39], [40]. 
Therefore, corrections have been reported in the literature and the derived expression from Miyatake et al.[39] is recommended to describe the full-stages of bubble growth within a superheated bulk liquid:

$$
r^{+}=\frac{2}{3}\left\{1+\frac{t^{+}}{3} \exp \left[-\left(t^{+}+1\right)^{1 / 2}\right]\right\}\left[\left(t^{+}+1\right)^{3 / 2}-\left(t^{+}\right)^{3 / 2}-1\right]
$$

where,

$$
\begin{gathered}
r^{+}=\frac{A}{B^{2}}\left[r(t)-r_{\text {crit }}\right] \\
t^{+}=\left(\frac{A}{B}\right)^{2}\left\{t-t_{g}\left[1-\exp \left[\left(-\frac{t}{t_{g}}\right)^{2}\right]\right]\right\} \\
A=\left[\frac{2}{3} \frac{\Delta P_{0}}{\rho_{l}}\right]^{1 / 2} \\
B=\left(\frac{12}{\pi}\right)^{1 / 2}\left\{\frac{\left[T_{l}-T_{\text {sat }}\left(P_{l}\right)\right] c_{p, l} \rho_{l} \alpha_{l}{ }^{1 / 2}}{i_{l v} \rho_{v}}\right\} \\
r_{\text {crit }}=\frac{2 \sigma}{\Delta P_{o}} \\
t_{g}=\frac{6 r_{\text {crit }}}{A} \\
\Delta P_{0}=P_{\text {sat }}\left(T_{l}\right)-P_{l}
\end{gathered}
$$

where, $t_{g}$ is the bubble growth delay period (s) and $\Delta P_{0}$ is the initial pressure difference between interior and exterior of a bubble (Pa). The correction of the Mikic [38] correlation by Miyatake [39] is illustrated in Fig. 7.

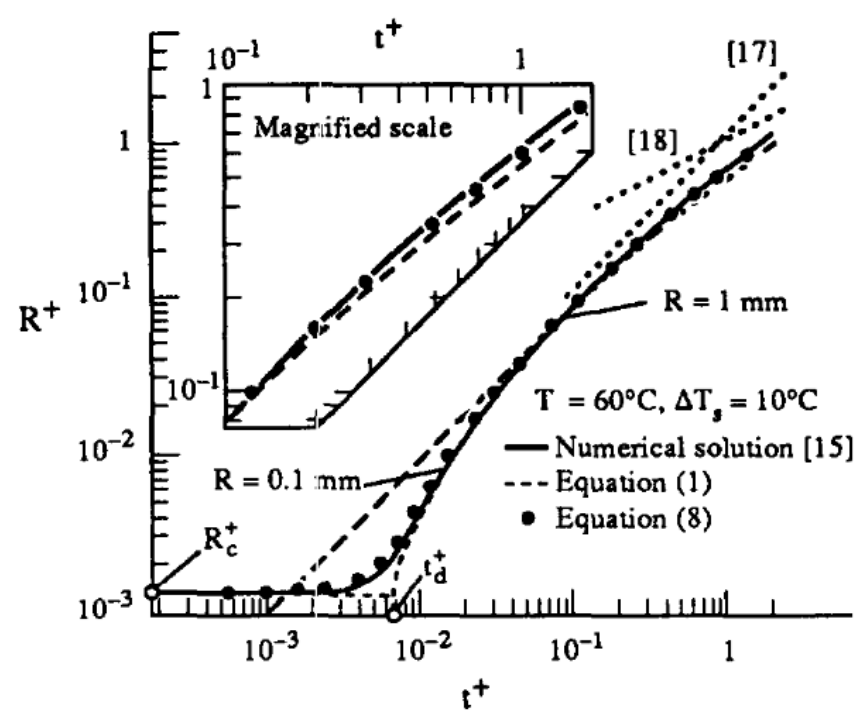

Figure 7: Bubble growth in a superheated bulk liquid (from Miyatake [39]), Comparison between Mikic [38] correlation (Equation 1), the corrected correlation from Miyatake [39] (Equation 8) and the numerical solution

It can be observed that the correction proposed by Miyatake [39] is relevant, in particular for $t^{+}$in the range $10^{-3}<t^{+}<10^{-1}$. The accuracy in the prediction of the bubble radius diameter for bubble growth in a superheated bulk liquid is demonstrated. Other correlations as proposed by Forster [41] and Zuber [42] to describe bubble growth from a superheated layer of liquid are in the form:

$$
r(t)=C J a(\alpha t)^{1 / 2}
$$


where, $C=\sqrt{\pi}$ for the Forster [41] correlation, $C=2 b / \sqrt{\pi}$ for the Zuber [42] correlation with $b$ a constant equal to 1 or $\pi / 2, \alpha$ is the thermal diffusivity $\left(\mathrm{m}^{2} \cdot \mathrm{s}^{-1}\right), t$ is the time (s) and $J a$ is the Jakob number defined by:

$$
J a=\frac{\left[T_{W}-T_{s a t}\left(P_{l}\right)\right] c_{p, l} \rho_{l}}{\rho_{v} i_{l v}}
$$

This form of correlation for bubble growth from a bulk liquid seems important to report as it was taken as a basis to develop models for bubble growth from surfaces.

In the case where the bubble is growing from a surface after a heterogeneous nucleation (which widely predominates in comparison to the bubble growth within the bulk liquid), the growing process differs as it is taking place near the cavity. Understanding the main mechanisms occurring in the heat transfer between the hot surface, the vapour bubble and the liquid is crucial in developing models predicting the bubble radius with time. After a heterogeneous nucleation, the bubble is initiated from the micro cavity. Then, the bubble will grow, detach from the cavity and be released into the bulk fluid. As the previous bubble has detached from the surface, a small portion of vapour is left inside the cavity which will generate the next bubble. Therefore, the bubble growth from a surface can be seen as a cycle. A model of bubble growth from a surface, adapted from Carey [24], is presented in Fig. 8-14.

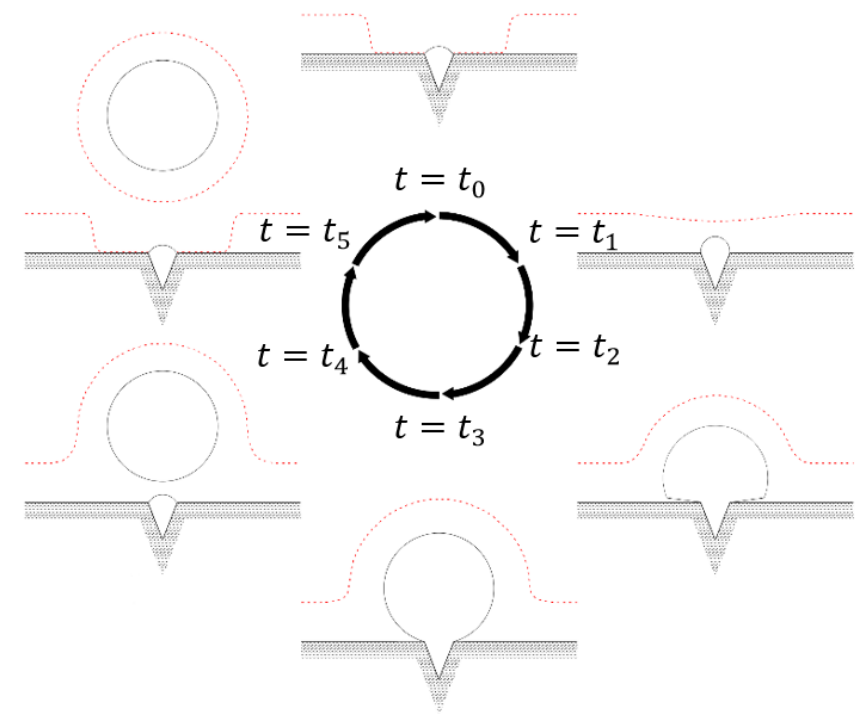

Figure 8: Cyclic model of bubble growth from a surface, adapted from Carey [24]

Each stage of the bubble growth is detailed hereafter. At $t=t_{0}$, the previous bubble has just left the surface. The thermal layer, defined as the liquid portion near the hot surface where the temperature is significantly higher than the bulk liquid due to its proximity with the heated surface, has been partially removed by the departing bubble. Moreover, a small portion of vapour has been left behind and will initiate the next heterogeneous nucleation process as shown in Fig. 9.

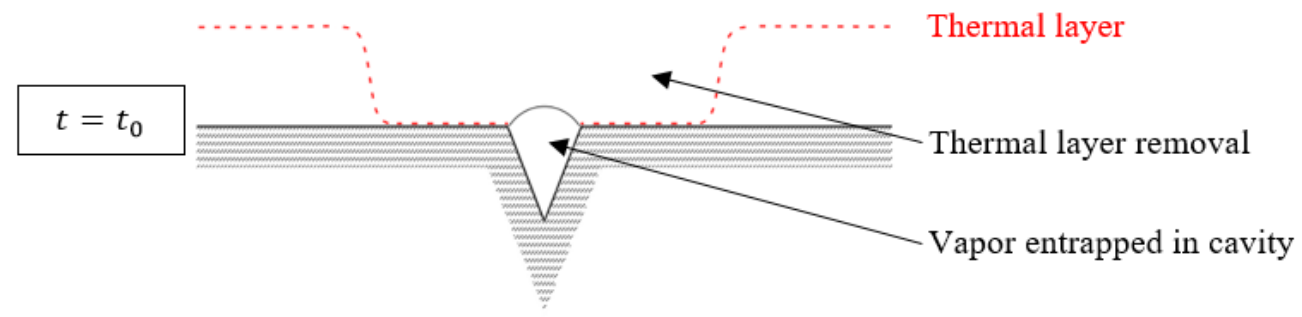

Figure 9: Bubble growth from a surface, stage $\mathrm{t}=\mathrm{t} 0$ 
For a period of time $t_{w}$ called "waiting period", the bubble is not growing as the thermal layer needs to be reformed. Liquid from the bulk enters into contact with the hot surface and starts to warm up by transient conduction, thus forming the superheated thermal layer until $t=t_{1}$ as illustrated in Fig. 10.

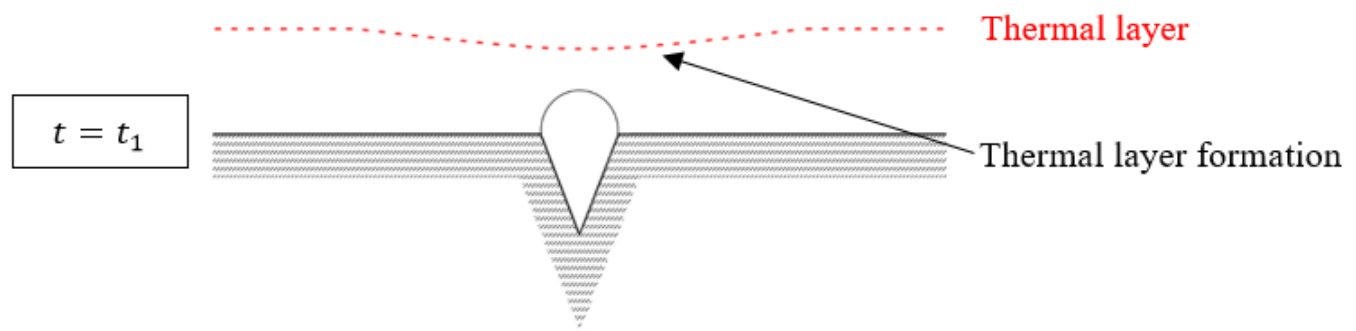

Figure 10: Bubble growth from a surface, stage $t=t 1$

As soon as the thermal layer has been rebuilt, the bubble starts to grow. At first, inertia-control growth occurs and a rapid increase of the bubble radius can be observed. However, the process is significantly eased in comparison with a growth within the bulk liquid due to the presence of a microlayer of liquid between the hot surface and the bubble. In addition, with the portion of liquid turning to vapour at the bubble-liquid interface, the evaporation of this very thin layer in the bubble significantly contributes to the increase in diameter of the bubble. This is schematized in Fig. 11.

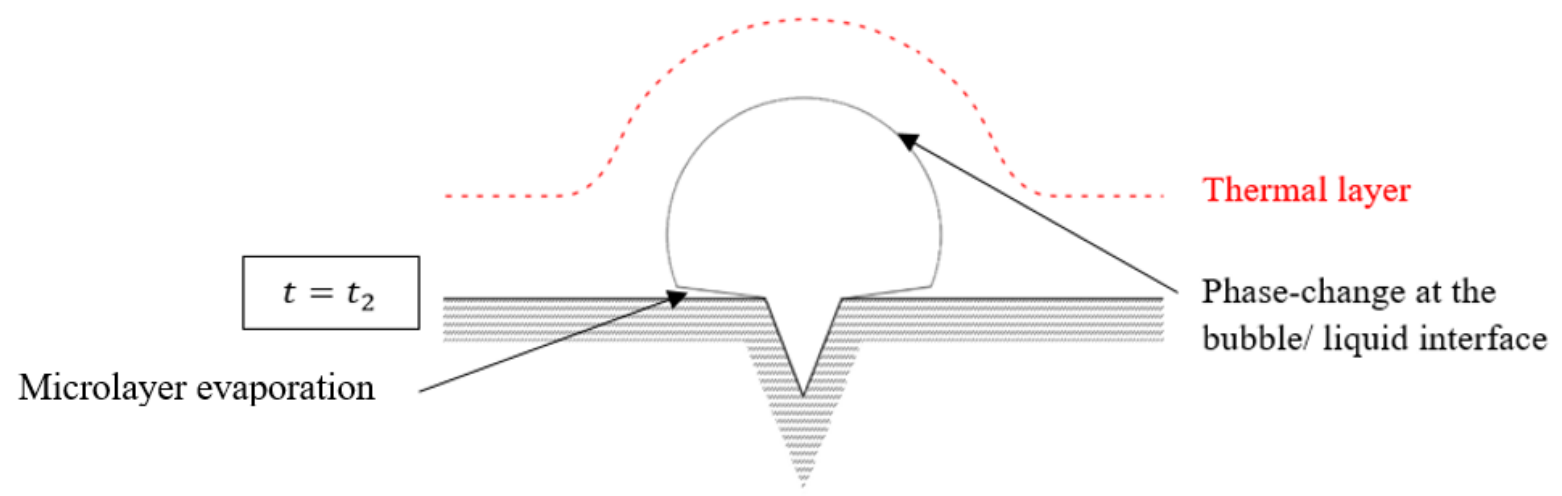

Figure 11: Bubble growth from a surface, stage $t=t 2$

Second, heat-transfer growth takes place and the bubble forms in a spherical shape as in Fig. 12.

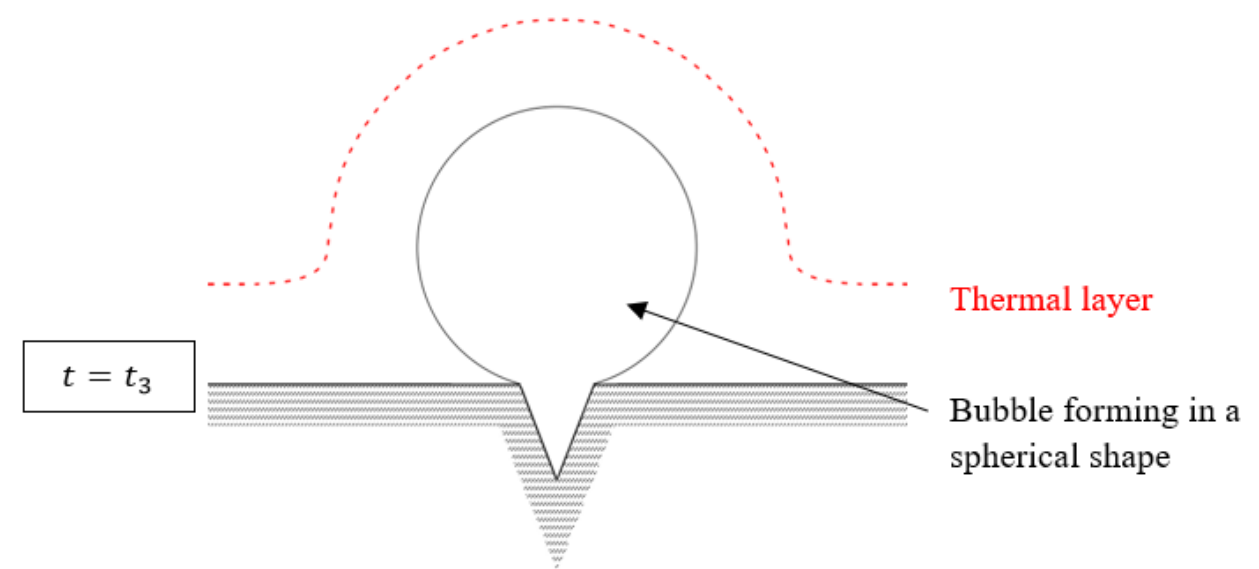

Figure 12: Bubble growth from a surface, stage $t=t 3$ 
At the departure time $t=t_{4}=t_{d}$, the bubble detaches from the surface. The bubble departure diameter is designated as $d_{b}$. A small portion of vapour from the bubble remains entrapped in the cavity which will generate the next bubble by heterogeneous nucleation. This is represented in Fig. 13.

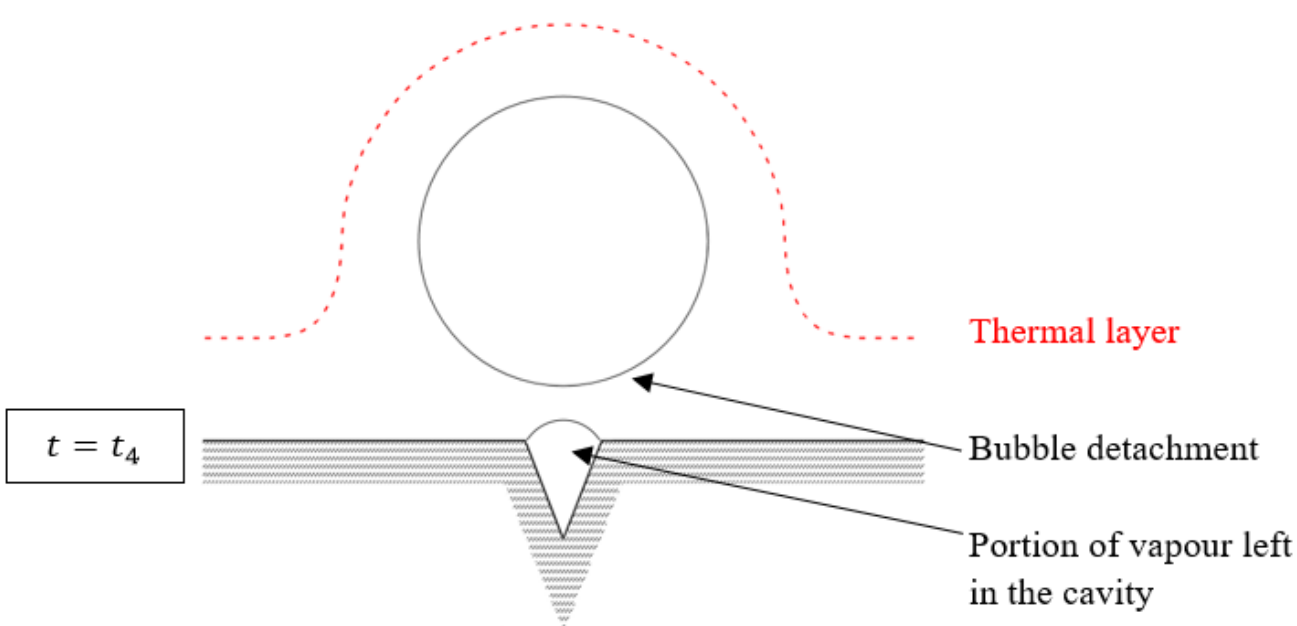

Figure 13: Bupdie growtn rrom a surrace, stage $\tau-\tau 4$

While departing from the hot surface, the bubble intensively enhances the boiling heat transfer. The study of this stage is critical in explaining why boiling heat transfer coefficients are the highest for nucleate boiling regimes, and how the presence of bubbles can improve the performance of a thermosyphon. To begin with, at the bubble departure, a portion of the thermal layer is removed and carried away by the bubble. Hence, this superheated portion of liquid mixes with the cooler bulk liquid. Second, the bubble serves as energy mover: the fluid is locally mixed by stirring action. This combined process is designated "locally enhanced convection" or "sensible heat transport". Then, as a part of the superheated thermal layer is removed, a portion of bulk liquid at lower temperature is able to reach the hot surface and rapidly warms up: this is known as "transient conduction". Finally, when the whole thermal layer carried away has mixed with the bulk liquid, heat transfer can occur between the bubble and the surrounding liquid. If the liquid is at a temperature lower than the saturation point, the bubble will yield its energy to the bulk liquid and turn to liquid. Therefore, energy is also carried by the bubble and can be transmitted to the surroundings by phase-change. This is referred as "latent heat transport". The three described phenomena occurring at the departure of a bubble that are responsible of the enhanced boiling heat transfer coefficients in nucleate boiling are shown in Fig. 14.

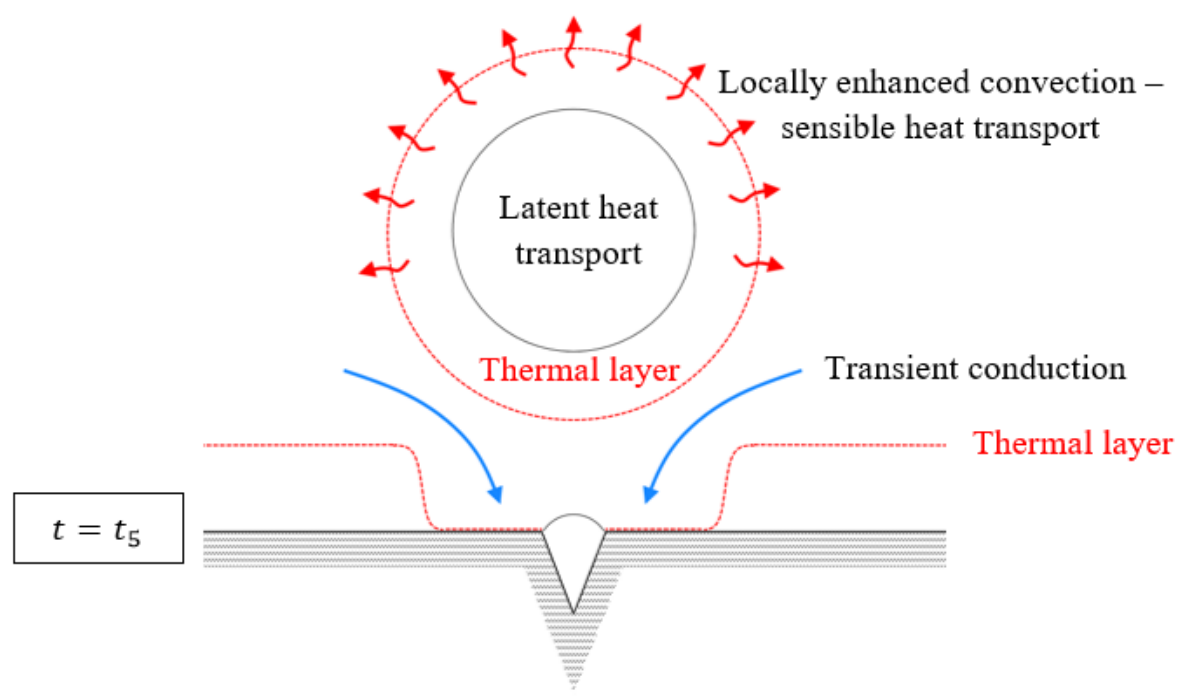

Figure 14: Bubble growth from a surface, stage $t=t 5$ 
Finally, the continuum process of bubble formation can restart and the next bubble formation will be initiated by heterogeneous nucleation. Mikic and Rohsenow [43] were among the first researchers to develop bubble growth correlations from a surface. However, their model considering only heattransfer-controlled growth is not recommended as the microlayer evaporation is neglected. Being inspired by the correlations developed for bubble growth from a superheated layer of liquid, Cole [44] developed the following empirical equation for a bubble growth from a surface:

$$
r(t)=\frac{5}{2} J a^{3 / 4}(\alpha t)^{1 / 2}
$$

Similarly, Cooper [45] proposed:

$$
r(t)=\frac{5}{2} \frac{J a}{P r_{l}^{0.5}}(\alpha t)^{1 / 2}
$$

These correlations are taken as references for a bubble growth from a surface as they consider the evaporation of the microlayer beneath the bubble. Moreover, their simple form makes them convenient to use.

Thus, by coupled phenomena of sensible heat transport, latent heat transport and transient conduction, the departures of bubbles allow a high transfer of energy between the hot surface and the hot liquid. For this reason, parameters dealing with the bubble departure, such as the departure diameter and frequency, are important factors to be determined when predicting nucleate boiling heat transfer.

\subsection{Bubble release diameter and frequency}

The estimation of the bubble diameter $D_{d}$ at departure from a surface is one of the most studied areas of boiling and the number of correlations reported is as high as for nucleate boiling correlations. The introduction of the dimensionless Bond number is relevant for this area of study:

$$
B_{o}=\frac{g\left(\rho_{l}-\rho_{g}\right) D_{d}^{2}}{\sigma}
$$

where $\mathrm{g}$ is the gravitational acceleration $\left(\mathrm{m} . \mathrm{s}^{-2}\right), \rho_{l}$ and $\rho_{v}$ are the liquid and vapor density, $D_{d}$ is the bubble departure diameter $(\mathrm{m})$ and $\sigma$ is the surface tension $\left(\mathrm{N} \cdot \mathrm{m}^{-1}\right)$. By doing a force balance between the buoyancy force and the surface tension of the bubble, Fritz [46] showed the following proportionality:

$$
D_{d} \propto \theta \sqrt{\frac{\sigma}{g\left(\rho_{l}-\rho_{v}\right)}}
$$

where $\theta$ is the contact angle $\left(^{\circ}\right)$. Then, Fritz [46] proposed the equation:

$$
D_{d}=0.0208 \times \theta \sqrt{\frac{\sigma}{g\left(\rho_{l}-\rho_{v}\right)}}
$$

where $\theta=35^{\circ}$ for mixtures and $\theta=45^{\circ}$ for water. This equation is the reference in the development of correlations to predict the bubble departure diameter. Many other correlations to estimate the bubble departure diameter $D_{d}$ for pure liquids have since been developed and some are listed in Table 2. 
Table 2: Bubble departure diameter $D_{d}$ correlations - State of the Art

\begin{tabular}{|c|c|c|c|}
\hline Authors & Correlation & $\begin{array}{c}\text { Frequency } \\
\text { of use }\end{array}$ & Year \\
\hline Fritz [46] & $D_{d}=0.0208 \times \theta \sqrt{\frac{\sigma}{g\left(\rho_{l}-\rho_{v}\right)}}$ & Very high & 1935 \\
\hline $\begin{array}{l}\text { Staniszewski } \\
\text { [47] }\end{array}$ & $D_{d}=0.0071 \times\left[1+\left(0.435 \frac{d D}{d t}\right)\right] \times \theta \sqrt{\frac{\sigma}{g\left(\rho_{l}-\rho_{v}\right)}}$ & Low & 1959 \\
\hline $\begin{array}{l}\text { Rukenstein } \\
{[48]}\end{array}$ & $D_{d}=\left[\frac{3 \pi^{2} \rho_{l} \alpha_{l}^{2} g^{1 / 2}\left(\rho_{l}-\rho_{v}\right)^{1 / 2}}{\sigma^{3 / 2}}\right]^{1 / 3} J a^{4 / 3} \sqrt{\frac{2 \sigma}{g\left(\rho_{l}-\rho_{v}\right)}}$ & Medium & 1961 \\
\hline $\begin{array}{l}\text { Cole and } \\
\text { Shulman [49] }\end{array}$ & $D_{d}=\frac{1000}{P} \sqrt{\frac{\sigma}{g\left(\rho_{l}-\rho_{v}\right)}}$ & Medium & 1966 \\
\hline Cole [50] & $D_{d}=0.04 \times \mathrm{Ja} \sqrt{\frac{\sigma}{g\left(\rho_{l}-\rho_{v}\right)}}$ & Very high & 1967 \\
\hline $\begin{array}{l}\text { Cole and } \\
\text { Rohsenow [51] }\end{array}$ & $\begin{array}{l}\qquad D_{d}=\mathrm{C}\left(J a^{*}\right)^{5 / 4} \sqrt{\frac{\sigma}{g\left(\rho_{l}-\rho_{v}\right)}} \\
\text { where, } \\
\qquad\left\{\begin{array}{c}C=1.5 \times 10^{-4} \text { for water } \\
C=4.65 \times 10^{-4} \text { for other fluids }\end{array}\right. \\
\qquad J a^{*}=\frac{c_{p, l} \rho_{l} T_{s a t}}{\rho_{v} i_{l v}} \text { is the modified Jakob number }\end{array}$ & High & 1969 \\
\hline $\begin{array}{l}\text { Stralen et al. } \\
\text { [52] }\end{array}$ & $D_{d}=2.63\left(\frac{J a^{2} \alpha_{l}^{2}}{g}\right)^{1 / 3}\left[1+\left(\frac{2 \pi}{3 J a}\right)^{1 / 2}\right]^{1 / 4}$ & Medium & 1978 \\
\hline $\begin{array}{l}\text { Golorin et al. } \\
\text { [53] }\end{array}$ & $\begin{aligned} D_{d} & =\frac{1.65 d^{*} \sigma}{g\left(\rho_{l}-\rho_{v}\right)}+\left[\frac{15.6 \rho_{l}}{g\left(\rho_{l}-\rho_{v}\right)}\right]^{1 / 3}\left[\frac{\beta_{d} k_{l}\left(T_{W}-T_{s a t}\right)}{\rho_{v} i_{l v}}\right]^{2 / 3} \\
\text { where, } &$\[ \begin{aligned} * d^{*} & =6 \times 10^{-3} \mathrm{~mm} \\
* \beta_{d} & =6 \text { for water, alcohol and benzene }\end{aligned} \]$\end{aligned}$ & Medium & 1978 \\
\hline $\begin{array}{l}\text { Kutateladze } \\
\text { and Gogonin } \\
\text { [54] }\end{array}$ & $\begin{array}{l}\qquad D_{d}=0.25\left(1+10^{5} K_{l}\right)^{1 / 2} \sqrt{\frac{\sigma}{g\left(\rho_{l}-\rho_{v}\right)}} \\
\text { where, } \\
\qquad K_{l}=\left(\frac{J a}{P r_{l}}\right)\left\{\left[\frac{g \rho_{l}\left(\rho_{l}-\rho_{v}\right)}{\mu_{l}}\right]\left[\frac{\sigma}{g\left(\rho_{l}-\rho_{v}\right)}\right]^{3 / 2}\right\}^{-1}\end{array}$ & High & 1979 \\
\hline
\end{tabular}




\begin{tabular}{|c|c|c|c|}
\hline $\begin{array}{l}\text { Kocamustafao } \\
\text { gu-llari }[55]\end{array}$ & $D_{d}=2.64 \times 10^{-5}\left(\frac{\rho_{l}-\rho_{v}}{\rho_{l}}\right)^{0.9} \sqrt{\frac{\sigma}{g\left(\rho_{l}-\rho_{v}\right)}}$ & Medium & 1983 \\
\hline $\begin{array}{l}\text { Gorenflo et al. } \\
\text { [56] }\end{array}$ & $D_{d}=C\left(\frac{J a^{4} \alpha_{l}^{4}}{g}\right)^{1 / 3}\left[1+\left(1+\frac{2 \pi}{3 J a}\right)^{1 / 2}\right]^{4 / 3}$ & Low & 1986 \\
\hline $\begin{array}{l}\text { Jensen and } \\
\text { Memmel [57] }\end{array}$ & $\begin{array}{l}\qquad D_{d}=0.19\left(1.8+10^{5} K_{l}\right)^{2 / 3} \sqrt{\frac{\sigma}{g\left(\rho_{l}-\rho_{v}\right)}} \\
\text { where, } \\
\qquad K_{l}=\left(\frac{J a}{P r_{l}}\right)\left\{\left[\frac{g \rho_{l}\left(\rho_{l}-\rho_{v}\right)}{\mu_{l}}\right]\left[\frac{\sigma}{g\left(\rho_{l}-\rho_{v}\right)}\right]^{3 / 2}\right\}^{-1}\end{array}$ & High & 1986 \\
\hline $\begin{array}{l}\text { Stephan and } \\
\text { Wenzel [58] }\end{array}$ & $D_{d}=0.25\left[1+\left(\frac{J a}{P r}\right)^{2} \frac{100000}{A r}\right]^{1 / 2} \sqrt{\frac{2 \sigma}{g\left(\rho_{l}-\rho_{v}\right)}}$ & Medium & 1992 \\
\hline $\begin{array}{l}\text { Zeng et al. } \\
\text { [59] }\end{array}$ & $\begin{array}{l}\qquad D_{d}=2\left\{\frac{3}{4} \frac{K^{2 / n}}{g}\left[\frac{3}{2} C n^{2}+n(n-1)\right]\right\}^{n /(2-n)} \\
\text { where, } \\
\quad C=20 / 3 \text { determined experimentally } \\
* K \text { and } n \text { empirical constants }\end{array}$ & Medium & 1993 \\
\hline $\begin{array}{l}\text { Yang et al. } \\
{[60]}\end{array}$ & $\begin{array}{l}\qquad D_{d}=3.0557 \times 10^{3} \frac{\rho_{l}}{\rho_{v}} \frac{\sqrt{c_{p, l} T_{s a t}}}{i_{l v}} \frac{\alpha_{l} P r_{l}^{3 / 5}}{\eta} \\
\text { where, } \\
\qquad \quad \eta=\psi J a^{0.3} \\
* \psi \text { a modified factor }\end{array}$ & Medium & 2000 \\
\hline Lee et al. [61] & $D_{d}=\left(50 \sqrt{27} J a \alpha_{l} \sqrt{\rho_{l} / \sigma}\right)^{2}$ & Low & 2003 \\
\hline $\begin{array}{l}\text { Kim and Kim } \\
{[62]}\end{array}$ & $D_{d}=0.1649 \times \mathrm{Ja}^{0.7} \sqrt{\frac{\sigma}{g\left(\rho_{l}-\rho_{v}\right)}}$ & Low & 2006 \\
\hline $\begin{array}{l}\text { Phan et al. } \\
\text { [63] }\end{array}$ & 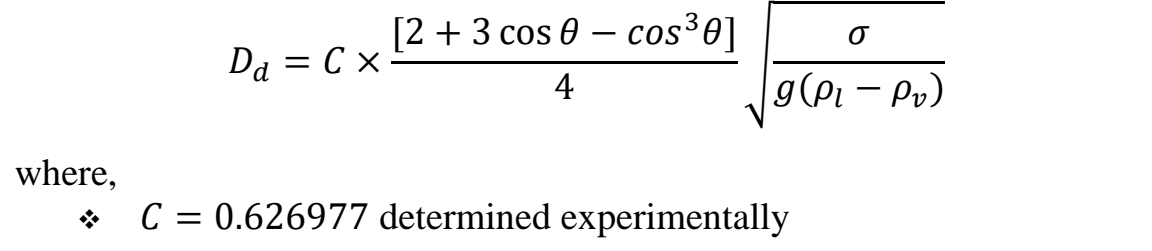 & Medium & 2009 \\
\hline $\begin{array}{l}\text { Phan et al. } \\
\text { [64] }\end{array}$ & $D_{d}=\left(6 \sqrt{\frac{3}{2}}\right)^{1 / 3}\left(\frac{\rho_{l}}{\rho_{v}}\right)^{-1 / 2}\left(\frac{\rho_{l}}{\rho_{v}}-1\right)^{1 / 3}(\tan \theta)^{-1 / 6} \sqrt{\frac{\sigma}{g\left(\rho_{l}-\rho_{v}\right)}}$ & Low & 2010 \\
\hline Nam et al. [65] & $D_{d}=\sqrt{\frac{24 \sin ^{2} \theta}{2+3 \cos \theta-\cos ^{3} \theta}} \sqrt{\frac{\sigma}{g\left(\rho_{l}-\rho_{v}\right)}}$ & High & 2011 \\
\hline $\begin{array}{l}\text { Lamas et al. } \\
{[66]}\end{array}$ & $D_{d}=\sqrt{0.0027\left(\frac{\rho_{l} c_{p, l} \Delta T}{\rho_{v} i_{l v}}\right)^{0.148}\left(\frac{\rho_{l}}{\rho_{v}}\right)^{-0.024} \exp (0.027 \times \theta)\left(\frac{\sigma}{g\left(\rho_{l}-\rho_{v}\right)}\right)}$ & Low & 2012 \\
\hline
\end{tabular}




\begin{tabular}{|c|c|c|c|}
\hline $\begin{array}{l}\text { Hamzekhani et } \\
\text { al. [67] }\end{array}$ & 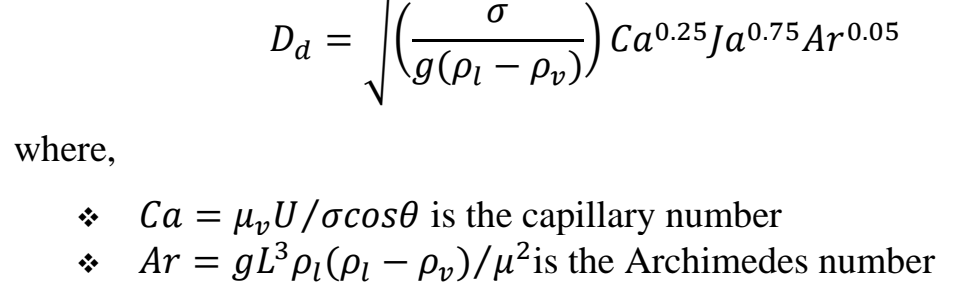 & Low & 2014 \\
\hline $\begin{array}{l}\text { Suszko and El- } \\
\text { Genk [68] }\end{array}$ & $\begin{array}{l}\qquad D_{d}=234+81 \sqrt{t_{b}} \text { for smooth surface } \\
\qquad D_{d}=206+48 \sqrt{t_{b}} \text { for rough surface } \\
\text { where, } t_{b} \text { is the bubble growth period }\end{array}$ & Low & 2015 \\
\hline $\begin{array}{l}\text { Bovard et al. } \\
\text { [69] }\end{array}$ & $\begin{array}{l}\quad D_{d}=C_{0}\left[C_{1}+J a^{C_{2}}(C a)^{C_{3}}\left(\frac{\alpha_{f}}{\alpha_{s}}\right)^{C_{4}}\right] \sqrt{\frac{\sigma}{g\left(\rho_{l}-\rho_{v}\right)}} \\
\text { where, } \\
\qquad \quad C a=\mu_{v} U / \sigma \cos \theta \text { is the capillary number } \\
* C_{0}=17.952177 \\
* C_{1}=0.0172742 \\
* C_{2}=1.285607 \\
* C_{3}=0.661205 \\
* C_{4}=0.025346\end{array}$ & Low & 2017 \\
\hline
\end{tabular}

One must be aware this list is only partial. In particular, correlations using the boiling heat flux were discarded, as the boiling heat flux participating in a single bubble detachment is supposed unknown from thermosyphons' users. In addition, correlations using a time dimension and considering the bubble growth rate are not considered. In most of the cases, these parameters are also not known from TPCTs users. The following analysis deals with the case of pure fluids only. To determine the most suitable correlation to use in the case of thermosyphons, a large number of references were studied. Indeed, as high discrepancies can occur during boiling experiments, it seems more relevant to base conclusions on a quantitative amount of works rather than on one set of experiments. Based on the references [21], [62], [67], [70]-[75] investigating the different correlations reported, the following recommendations are made to predict the departure diameter of a bubble from a surface: The correlation from Jensen and Memmel [57] is firstly recommended as it combines an important accuracy with a relatively simple form. This correlation is a modified version of the Kutateladze and Gogonin [54] correlation that was established using a large set of data. Its reliability has therefore been proved for more than twenty years. The Cole [50] correlation is the second correlation advised as it has been widely used. Its limits are known, in particular, the value of the constant can be discussed. Yet, according to the work reported in the literature, data are globally in a good agreement with this correlation and show an accuracy within $\pm 30 \%$. Unlike expectations, the corrected form of the Cole [50] correlation proposed by Cole and Rohsenow [51] is not advised. Despite the relevant introduction of the corrected Jakob number to solve the discrepancy between experimental and theoretical data regarding the non-proportionality between the departure diameter of the bubble and the wall superheat, the correlation by Cole and Rohsenow [51] is mainly reported as inaccurate and it often overestimates the departure diameter. A promising recent work observed is the correlation by Hamzekhani et al. [67] that was recently reused by some authors [35], [76] and seems to fit quite well to other sets of experimental data. Yet, as this correlation is recent, its reliability remains to be tested on a wider scale. Therefore, the use of this correlation must then be made cautiously. At last, the bubble departure diameter can be estimated with the Fritz [46] correlation 
with a decent accuracy in cases where making an error in the bubble release diameter $D_{d}$ is not a big issue. The summation of the analysis on bubble release diameter $D_{d}$ is presented visually in Table 3.

Table 3: Bubble departure diameter $D_{d}$ correlations - Recommendations

\begin{tabular}{|c|c|c|c|c|}
\hline Authors & $\begin{array}{c}\text { Frequency } \\
\text { of use }\end{array}$ & Year & Comments & $\begin{array}{l}\text { Recomm } \\
\text { endation }\end{array}$ \\
\hline $\begin{array}{l}\text { Jensen and } \\
\text { Memmel [57] }\end{array}$ & High & 1986 & $\begin{array}{l}\text { Good accuracy for a relatively simple form } \\
\text { Modified version of Kutateladze and Gogonin [54] } \\
\text { correlation established using a large set of data. } \\
\text { Reliability proved for more than } 30 \text { years }\end{array}$ & 1 \\
\hline Cole [50] & Very High & 1967 & $\begin{array}{l}* \quad \text { Decent accuracy for a relatively simple form } \\
* \quad \text { The most used correlation after Fritz [46] } \\
* \quad \text { Also suitable for mixtures } \\
\end{array}$ & 2 \\
\hline $\begin{array}{l}\text { Hamzekhani et } \\
\text { al. [67] }\end{array}$ & Low & 2014 & $\begin{array}{l}* \quad \text { Estimated accuracy of } 30 \% \\
* \quad \text { Promising recent correlation } \\
\text { * } \\
\text { Reliability to be proved for a higher number of } \\
\text { experiments } \\
\text { * To be used with caution }\end{array}$ & 3 \\
\hline Fritz [46] & Very high & 1935 & $\begin{array}{ll}* & \text { Basic correlation } \\
* \quad \text { Simplest form } \\
* \quad \text { To be used when an absolute accuracy is not required }\end{array}$ & 4 \\
\hline
\end{tabular}

It must be noticed that the new computational progress made, leading to higher calculation power, is opening new opportunities to understand better the phenomena implied in the bubble growth and release from a surface. In particular, the temperature distribution and the evaporation of the microlayer beneath the bubble are the focus of recent computational studies that lead to new correlations. This can, for instance, be illustrated by the works of Nam et al. [65], Lamas et al. [66], Dhir [74] or Stephan and Fuchs [77].

When estimating the boiling heat transfer, the rate of bubble departure is also of interest. Similar to the bubble departure diameter, numerous correlations have been presented to estimate the average bubble departure frequency $f_{d}$. As previously discussed, the bubble formation from a surface is divided between a bubble growth period $t_{g}$ and a waiting period $t_{w}$. During the bubble growth period $t_{g}$, the nucleation process occurs, followed by the growing of the bubble until its departure. After the departure of the bubble, a period during which the thermal layer needs to be rebuilt is observed and designated as the waiting period $t_{w}$. These two periods can be observed in the work by Collier and Thome [78] where the influence of these two periods on the wall temperature is studied. This is illustrated in Fig. 15. 


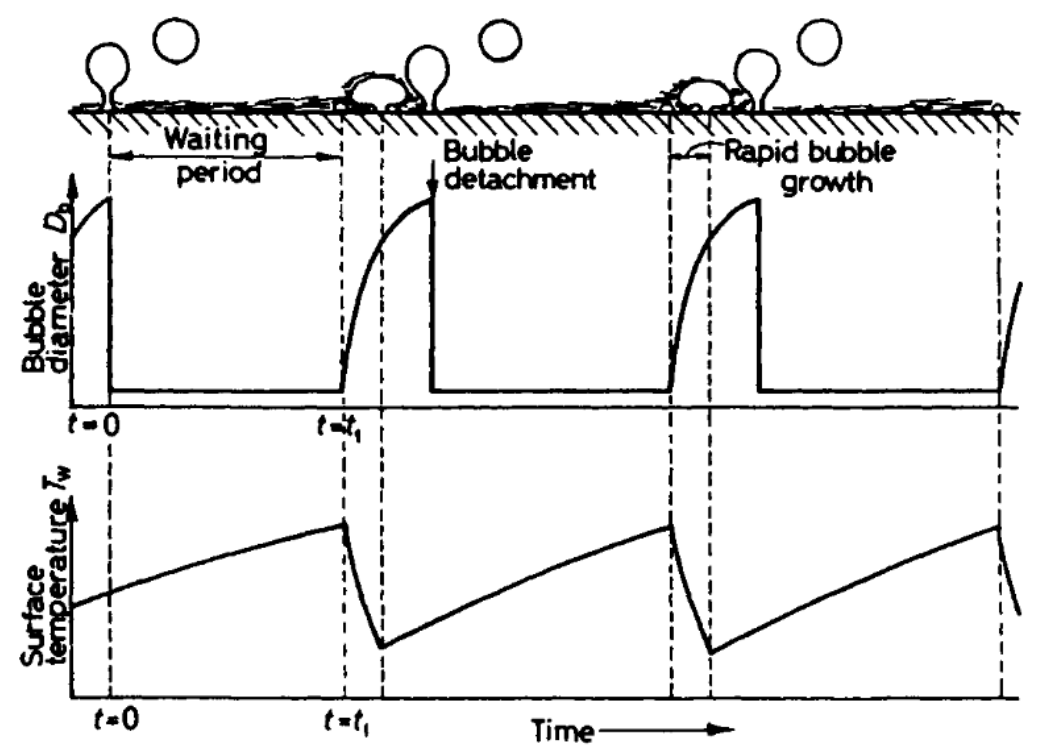

Figure 15: Bubble departure frequency, bubble growth period and waiting period, from Collier and Thome [78] Hence, the bubble departure frequency $f_{d}$ can be expressed by [21]:

$$
f_{d}=\frac{1}{t_{w}+t_{g}}
$$

As the bubble growth time $t_{g}$ is directly linked to the bubble departure diameter, it can be concluded from this expression that the bubble departure frequency $f_{d}$ is related to the bubble departure diameter $D_{d}$. Therefore, most of the correlations reported in the literature to evaluate the bubble departure frequency $f_{d}$ also include the bubble departure diameter as a parameter. In particular, Ivey [79] asserts that the product $f_{d}^{2} D_{d}$ should be constant for a bubble departure governed by inertiacontrolled growth while the product $f_{d}{ }^{1 / 2} D_{d}$ should be constant for a bubble departure governed by heat-transfer-controlled growth. Therefore, the bubble departure frequency $f_{d}$ is mainly correlated as:

$$
f_{d} D_{d}{ }^{m}=f\left(\rho_{l}, \rho_{v}, g, \sigma, \alpha_{l}, J a\right)
$$

Some of the correlations reported in the literature are reviewed in Table 4.

Table 4: Bubble departure frequency $f_{d}$ correlations - State of the Art

\begin{tabular}{|l|c|c|c|}
\hline \multicolumn{1}{|c|}{ Authors } & Correlation & $\begin{array}{c}\text { Frequency } \\
\text { of use }\end{array}$ & Year \\
\hline $\begin{array}{l}\text { Jakob and } \\
\text { Fritz [80] }\end{array}$ & $f_{d} D_{d}=0.078$ & High & 1931 \\
\hline & $f_{d} D_{d}=U_{b}\left[\frac{t_{g}}{t_{w}+t_{g}}\right]$ & & \\
$\begin{array}{l}\text { Jakob and } \\
\text { Linke [81] }\end{array}$ & $\begin{array}{r}\text { where, } \\
* t_{g} \text { is the bubble growth period }\end{array}$ & High & 1933 \\
& $* t_{w}$ is the waiting period & & \\
\hline Jakob [82] & $U_{b}$ is the bubble velocity at departure & Very high & 1949 \\
\hline
\end{tabular}




\begin{tabular}{|c|c|c|c|}
\hline $\begin{array}{l}\text { Peebles and } \\
\text { Garber [83] }\end{array}$ & $f_{d} D_{d}=1.18\left[\frac{t_{g}}{t_{w}+t_{g}}\right]\left[\frac{\sigma g\left(\rho_{l}-\rho_{v}\right)}{\rho_{l}^{2}}\right]^{1 / 4}$ & High & 1953 \\
\hline Cole [84] & $f_{d} D_{d}^{1 / 2}=\left[\frac{4 g\left(\rho_{l}-\rho_{v}\right)}{3 \rho_{l}}\right]^{\frac{1}{2}}$ & Very high & 1960 \\
\hline $\begin{array}{l}\text { McFadden and } \\
\text { Grassmann } \\
\text { [85] }\end{array}$ & $f_{d} D_{d}^{1 / 2}=0.56 g^{1 / 2}$ & Medium & 1962 \\
\hline Zuber [86] & $f_{d} D_{d}=\left(\frac{1.18}{2}\right)\left[\frac{\sigma g\left(\rho_{l}-\rho_{v}\right)}{\rho_{l}^{2}}\right]^{1 / 4}$ & High & 1963 \\
\hline $\begin{array}{l}\text { Hatton and } \\
\text { Hall [87] }\end{array}$ & $\begin{array}{l}\qquad f_{d} D_{d}{ }^{2}=284.7 \alpha_{l} \\
\text { where, } \\
\qquad \alpha_{l} \text { is the liquid thermal diffusivity }\end{array}$ & Low & 1966 \\
\hline Ivey [79] & $\left\{\begin{array}{c}f_{d} D_{d}^{1 / 2}=0.9 g^{1 / 2} \text { for hydrodynamic region } \\
f_{d} D_{d}^{3 / 4}=0.44 g^{1 / 2} \text { for transition region } \\
f_{d} D_{d}^{2}=C \text { for thermodynamic region }\end{array}\right.$ & High & 1967 \\
\hline $\begin{array}{l}\text { Mikic and } \\
\text { Rohsenow [43] }\end{array}$ & $\left\{\begin{array}{c}f_{d}^{1 / 2} D_{d}=\left(\frac{4}{\pi}\right) J a \sqrt{3 \pi \alpha_{l}}\left[\left(\frac{t_{g}}{t_{w}+t_{g}}\right)^{\frac{1}{2}}+\left(1+\frac{t_{g}}{t_{w}+t_{g}}\right)^{\frac{1}{2}}-1\right] \\
f_{d}^{1 / 2} D_{d}=0.83 \mathrm{Ja} \sqrt{\pi \alpha_{l}} \text { for } 0.15<\frac{t_{g}}{t_{w}+t_{g}}<0.8\end{array}\right.$ & High & 1969 \\
\hline Stephan [88] & $f_{d} D_{d}=\frac{1}{\pi}\left[\frac{g}{2}\left(D_{d}+\frac{4 \sigma}{\rho_{l} g D_{d}}\right)\right]^{\frac{1}{2}}$ & Low & 1992 \\
\hline $\begin{array}{l}\text { Kumada and } \\
\text { Sakashita [89] }\end{array}$ & $\begin{array}{l}\qquad f_{d}=0.215\left\{\left[\frac{g\left(\rho_{l}-\rho_{v}\right)}{\rho_{l}}\right]^{\frac{5}{9}} /\left(v_{l} D_{s}\right)^{\frac{1}{9}}\right\} \\
\text { where, } \\
\qquad v_{l} \text { is the liquid kinematic viscosity } \\
* D_{s} \text { is the heating surface diameter when a disk is considered }\end{array}$ & Low & 1995 \\
\hline Kim et al. [90] & $f_{d} D_{d}^{4.85}=7.2 \times 10^{-8}$ & Low & 2006 \\
\hline $\begin{array}{l}\text { Sakashita and } \\
\text { Ono [91] }\end{array}$ & $f_{d}=0.6\left[\frac{g\left(\rho_{l}-\rho_{v}\right)}{\rho_{l}}\right]^{\frac{2}{3}}\left\{v_{l}\left[\frac{g\left(\rho_{l}-\rho_{v}\right) \rho_{l}^{2} v_{l}^{4}}{\sigma^{3}}\right]^{-0.25}\right\}^{\frac{-1}{3}}$ & Low & 2009 \\
\hline
\end{tabular}

In accordance with the previous analysis of bubble departure diameter correlations, one must be aware that correlations using the heat flux have not been considered for similar reasons. In the analysis of nucleate pool boiling in thermosyphons, it is supposed that the heat flux per active nucleation site is not known. Based on the references [21], [35], [62], [73], [89], [92], the following conclusions and recommendations are made to estimate the average bubble departure frequency $f_{d}$ in a thermosyphon evaporator: A high discrepancy of data and correlation analysis can be observed within the literature. Therefore, it seems relevant to base the bubble departure frequency estimation on the reference correlations. The most recommended predictive model of bubble departure frequency is the Cole [84] correlation. This equation, obtained by balancing buoyancy and drag forces, is considered as a major advance in the study of bubble departure and it has been reused by authors to develop derived 
correlations. Its accuracy is decent in most of the cases and it is particularly suitable for water. Initially, this correlation was developed for inertia-controlled growth. Its applicability for heat-transfer controlled growth is to be questioned. The Jakob [82] correlation is the second correlation to consider when evaluating the average bubble departure frequency. As a result of preceding work, this simple form includes the thermo-physical properties of the working fluids and surface tension. By the promising dependence of the bubble departure frequency with this equation, the Jakob [82] correlation has been widely derived as a basis for new predictive models, as attested by the correlations proposed by Peebles and Garber [83] and Zuber [86]. Then, the model of Ivey [79] is advised. According to the author, three regions with different physical mechanisms can be identified: In the hydrodynamic region, the buoyancy and drag forces are predominant and the inertia, surface tension and viscous forces are neglected. A transition region, during which the forces of buoyancy, drag and surface tension are ruling the bubble departure, can be observed until the thermodynamic region takes place. In the thermodynamic region, both bubble diameter and frequency are governed by thermodynamic considerations such as heat-transfer-controlled growth [79]. In this analysis, Ivey [79] reports the previously discussed correlations from Cole [84] and Jakob [82] belonging to the hydrodynamic and transition region, respectively. Even if dividing the bubble departure frequency predictions into a hydrodynamic region and a thermodynamic region is not widely used in the literature, the similarities with the previously discussed nucleation processes and bubble growth, either controlled by inertia or heat transfer, proves the relevance of considering two regions governed by inertia forces or heat-transfer limits. In addition, the three correlations from Ivey [79] are based on a high number of data and compared to previous correlations which seem to prove the reliability of this model. Finally, the more recent work from Kumada and Sakashita [89] can be used to estimate the average frequency of bubble departure. This semi-empirical equation was derived by dimensional analysis of force balance equations. Yet, this correlation has not been widely used yet; its reliability must be tested on a wider scale by other authors to assess its accuracy. The correlations recommended to estimate the bubble departure frequency are presented in Table 5.

Table 5: Bubble departure frequency $f_{d}$ correlations - Recommendations

\begin{tabular}{|c|c|c|c|c|}
\hline Authors & $\begin{array}{c}\text { Frequency } \\
\text { of use }\end{array}$ & Year & Comments & $\begin{array}{l}\text { Recomm } \\
\text { endation }\end{array}$ \\
\hline Cole [84] & Very high & 1960 & $\begin{array}{l}\text { Reference widely used and derived } \\
\text { Decent accuracy } \\
\text { * Simple form } \\
\text { Based on a balance between buoyancy and drag } \\
\text { forces }\end{array}$ & 1 \\
\hline Jakob [82] & Very high & 1949 & $\begin{array}{l}\text { * Reference widely used and derived } \\
\text { * Sesult of preceding work } \\
\text { * Surface tension considered } \\
\text { * This correlation may be completed by another term } \\
\text { to increase the accuracy }\end{array}$ & 2 \\
\hline Ivey [79] & High & 1967 & $\begin{array}{l}\text { * } \quad \text { Based on a high number of data } \\
\text { * } \quad \text { Three regions considered: hydrodynamic, transition } \\
\text { and thermodynamic regions } \\
\text { * Similarities with nucleation and bubble growth } \\
\text { considerations that seem relevant }\end{array}$ & 3 \\
\hline
\end{tabular}




\begin{tabular}{|l|l|l|l|l|}
\hline $\begin{array}{l}\text { Kumada and } \\
\text { Sakashita [89] }\end{array}$ & Low & \multirow{4}{*}{1995} & $\begin{array}{l}* \begin{array}{l}\text { Developed using an important number of data } \\
\text { Compared with widely used references } \\
\end{array} \\
* \begin{array}{l}\text { Recent and not often used } \\
\text { Reliability to be proved by other authors }\end{array}\end{array}$ & 4 \\
\hline
\end{tabular}

Regardless of the recommendations made, one must be aware that a high discrepancy between the experiments reported in the literature and the conclusions of various authors is observed when it comes to estimating the bubble departure frequency. In opposition to the bubble departure diameter analysis where a better idea of the correlation accuracy was reported, researchers still struggle with estimating the average bubble departure frequency [79]. It is unclear that correlations present significant improvements compared to the references established more than fifty years ago by Jakob [82] and Cole [84]. In particular, when considering only the global fluid properties to estimate an average bubble departure frequency, factors such as heat flux, bubble shape, surface-liquid interface, surface aspect, roughness and cavities' shape and number are not considered. Yet, the micro-scale of the phenomena implied seems to rule the bubble departure process and therefore a global scale remains inaccurate and difficult to predict. Moreover, the interdependence of both bubble departure frequency and diameter increases the inaccuracy of predictive models. Therefore, by using up to date reported correlations, high errors (typically more than 50\%) are to be expected when predicting the bubble departure frequency.

\section{NUCLEATE POOL BOILING}

At the evaporator of a heat pipe, pool boiling is taking place. In the case where the thermosyphon's filling ratio is $100 \%$, pool boiling is the only resistance to consider when evaluating the performance of a TPCT. As briefly introduced, pool boiling refers to the phase change of stationary fluids in which no motion is imposed by external means. Different regimes of pool boiling can be observed depending on the heat flux imposed at the heating surface. The heat transfer capacity of a thermosyphon is also impacted by the flow regime in the pool. Hence, it is relevant to present the different pool boiling regimes and to understand the physical phenomena implied to identify the most efficient pool boiling regime. This brings information on the most suitable working conditions for heat pipes to increase their efficiencies. Finally, the analysis of pool boiling proves that the performance of a TPCT is directly linked to the boiling mechanism. Thus, improving boiling heat transfer leads to a significant improvement of thermosyphon efficiencies.

\subsection{Boiling curve and pool boiling regimes}

To start with, boiling is described as "subcooled" or "saturated" boiling, depending on the liquid bulk temperature. In subcooled boiling, the temperature of the volume in the pool is lower than the saturation point of the working fluid. It must be noted that, during subcooled pool boiling, a liquid layer close to the wall can be at the saturation point or above. Hence, bubbles can form at the wall surface but they will detach and collapse in the subcooled bulk. In saturated boiling, the whole pool volume is at the saturation temperature of the working fluid for the given pressure. Bubbles are formed near the wall and reach the liquid-gas interface of the pool. Thus, disturbances appear at the pool surface due to the rise and burst of bubbles. As introduced, different pool boiling regimes are observed regarding the input heat flux. These regimes are illustrated in Fig. 16 using a typical pool-boiling curve of water at 1 atm pressure, adapted from Yunus A. Cengel [20]. Yet, the trend is similar for all fluids. 


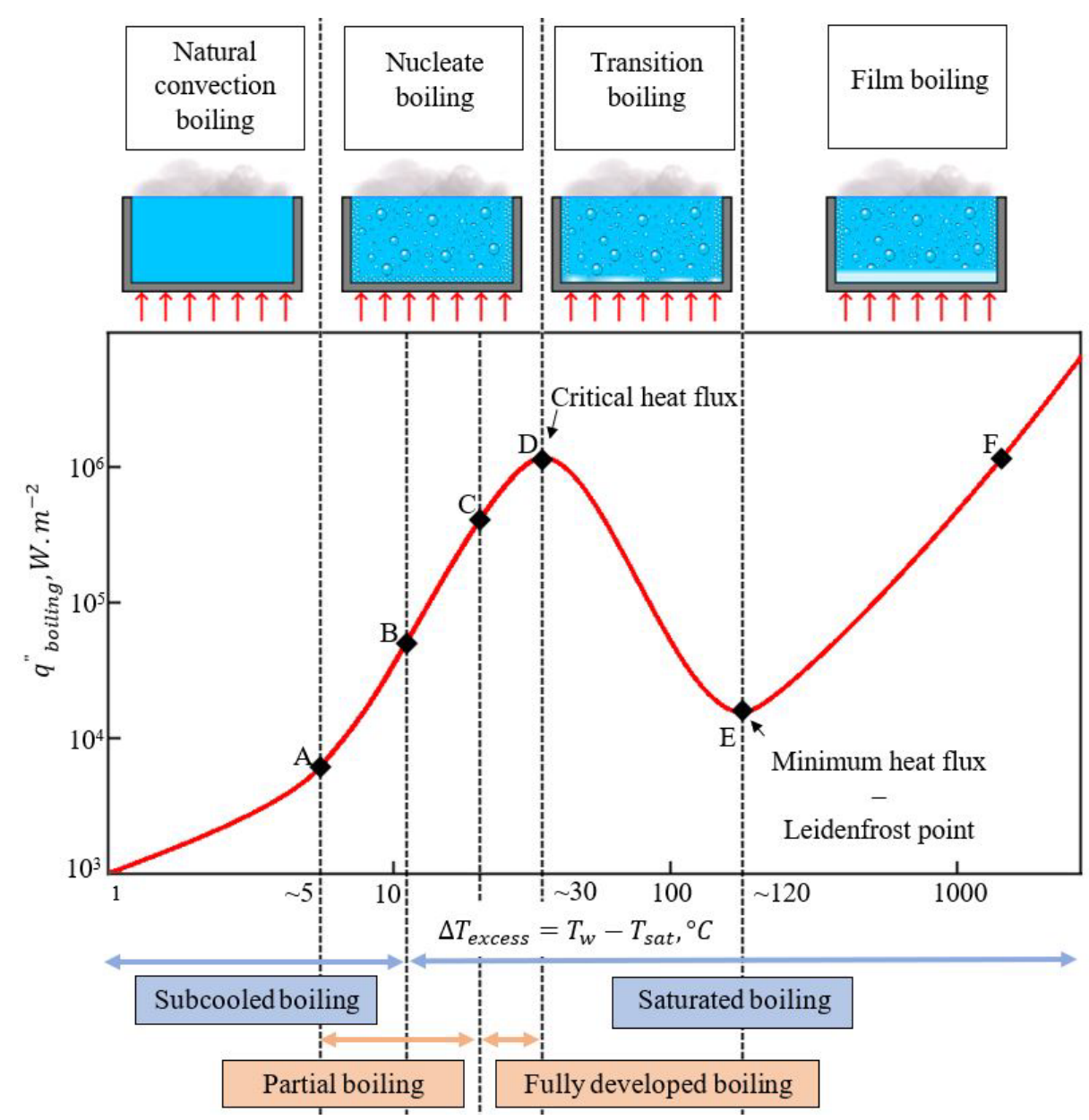

Figure 16: Pool boiling curve of water at 1 atm pressure, adapted from Yunus A. Cengel [20]

The pool-boiling curve presented describes the evolution of the boiling heat flux per unit area $q{ }_{\text {boiling }}$ with the wall superheat $\Delta T_{\text {excess }}$ which is the excess temperature between the wall and the saturation point of the working liquid. The following pool boiling regimes are observed:

\section{- Natural convection boiling (to point A)}

In natural convection boiling, the bulk liquid is subcooled. The wall temperature is only few degrees above the saturation point of the working fluid. Initially, no bubbles are observed. When increasing the excess temperature of the wall, bubbles may form near the evaporator wall but do not detach from the surface before a much higher bubble density is reached. The only motion of fluid observed in the pool is due to natural convection currents, where the local difference of fluid temperature due to the proximity of the heating surface may provoke a change in the fluid density and thus create motion. Because of its low boiling heat transfer coefficients, natural convection has received little attention [19].

\section{- Nucleate boiling (Point A to D)}

In practice, bubbles start to form and detach a few degrees above the fluid saturation point (typically around 2 to $6^{\circ} \mathrm{C}$ for water [20]). The departure of bubbles from the heating surface significantly enhances the boiling heat transfer. This regime is called nucleate boiling. Despite its complexity, nucleate boiling has been widely studied over the years as it presents the best heat transfer coefficients. Therefore, in the case of a thermosyphon, explaining and improving nucleate pool boiling leads to a direct improvement of the TPCT performance. Different nucleate boiling regions can be observed due to the appearance of various physical phenomena. To understand the link between these nucleate boiling mechanisms and the evolution of the nucleate boiling heat transfer coefficient, the notion of partial 
boiling/fully developed boiling must be introduced. As observed by authors such as Nishikawa [93] and Merte et al. [94], when a certain heat flux is reached, nucleate boiling inside the thermosyphon's pool becomes independent to several factors such as gravitational acceleration, liquid depth above the boiling surface or inclination angle and becomes governed by near-wall phenomena. This is assumed to be caused by the transition between partial boiling and fully developed boiling. In partial boiling, also known as the isolated bubble region, bubbles form at the hot surface, grow, detach and rise independently. Yet, after the transition to fully developed boiling, bubbles start to merge, forming stems of vapour that will lead to vapour mushrooms. This was explained by Gaertner [95] whose model, considered as a basis for many studies, is presented in Fig. 17.

During nucleate pool boiling, the following three regions can be observed:

- $\quad$ Subcooled and Partial nucleate boiling (Point A to B):

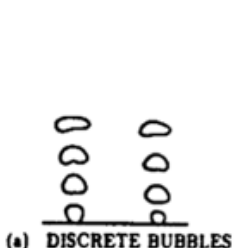

(a)

Figure 17: Vapour structures in nucleate boiling, from Gaertner [95]

Isolated bubbles (Fig. 17 (a)) are formed at the nucleation sites and detach from the surface but collapse in the subcooled bulk liquid. As discussed in the bubble section, the heat transfer is enhanced by the transient conduction, latent heat transport and sensible heat transport mechanisms generated by the departure of bubbles. The stirring action of bubbles also acts as an enhanced convection motion and participates in increasing the subcooled bulk liquid temperature.

- $\quad$ Saturated and Partial nucleate boiling (Point B to C):

At this stage, the bulk temperature has reached the saturation point. The isolated bubbles persist and reach the surface of the pool. With an increase of wall superheat, as the bubble departure frequency and diameter increase, stems of vapour start to form. At some point, these jets of vapour may merge and form small vapour mushrooms (Fig. 17 (b)). This is the transition between partial and fully developed boiling.

- Saturated and Fully developed nucleate boiling (Point C to D):

In saturated fully developed nucleate boiling, stems of vapour from adjacent nucleation sites merge and form large vapour mushrooms above a liquid layer called a macro-layer (Fig. 17 (c)). The presence of this liquid macro-layer underneath the vapour mushrooms may explain the near-wall phenomena observed after the transition to fully developed boiling. Then, boiling is governed by the evaporation of the near wall macro-layer. At high excess temperature (close to point $\mathrm{D}$ ), dry-out of the macro-layer may occur (Fig. 17 (d)) which tends to decrease the nucleate boiling heat transfer coefficient.

\section{- Critical heat flux CHF (Point D)}

While increasing the wall superheat, a maximum heat flux, namely critical heat flux (CHF), is reached and this represents the limit of nucleate boiling. Beyond this point, the pool boiling heat transfer decreases and the burnout phenomenon can occur. The burnout phenomenon was investigated by scientists, such as Nukiyama [96] in 1934 (translated in 1966), who observed the incapacity of the working fluid to receive more energy after the critical heat flux [97]. As a result, instead of being transferred to the working fluid, the energy supplied by the heater is absorbed by the heating surface itself, thus provoking a chain reaction resulting in a sudden rise of the heating surface temperature. The exact scientific mechanisms that are causing the transition to the critical heat flux point are not fully 
understood today. Four postulated models are proposed. The hydrodynamic instability model stipulates that instabilities at the vapour-liquid interface create an accumulation of vapour at the surface. The macro-layer consumption model assumes that, between the departures of two vapour mushrooms, the macro-layer situated underneath the mushroom has enough time to evaporate and is totally consumed. The bubble crowding at the heated surface model postulates that the CHF takes place when the important bubble coalescence near the surface reduces the amount of liquid in contact with the hot surface. Finally, the critical heat flux may be caused by hot-spot heating, meaning that local dry-out points cannot be rewetted due to the high wall temperature [21].

\section{- Transition boiling (Point D to E)}

Beyond the critical heat flux point, the boiling regime transits from nucleate to film boiling. As partial vapour film layers are forming at the solid-liquid interface, the pool boiling heat transfer resistance increases and the heat transfer coefficient is lower. Along the transition, the liquid fraction near the wall decreases and is almost null at the Leidenfrost point, also known as minimum heat flux (MHF). This is represented by point $\mathrm{E}$ in Fig. 16.

\section{- Film boiling (Point E to F)}

In film boiling, a complete vapour layer is formed at the surface-liquid interface. Beyond the MHF point, at high excess temperature, this vapour film blankets the hot surface and prevents contact between the liquid and the surface. Radiative heat transfer through the vapour layer becomes significant.

In a thermosyphon, the pool boiling regimes occurring at the evaporator are natural convection boiling and nucleate boiling. Natural convection typically occurs while the heat-pipe based unit, a heat exchanger for instance, has recently been started. Due to the potential high heat transfer coefficients, nucleate boiling is the regime wanted for heat pipes in order to transfer large amounts of heat. However, the critical heat flux, followed by the transition and film boiling regions, is to be avoided as it may damage the thermosyphon installation due to the burnout phenomenon and the appearance of dry patches.

Therefore, to reach higher performances, thermosyphons use nucleate pool boiling, as the action of bubbles permits better heat transfer coefficients to be achieved. Then, to estimate the thermal resistance of a thermosyphon, the nucleate pool-boiling coefficient must be predicted.

\subsection{Nucleate pool boiling heat transfer correlations}

Because of the complex phenomena involved such as nucleation and growth processes of a bubble, bubble departure diameter and frequency, coupled transient conduction with latent heat transport and sensible heat transport, formation of vapour stems and consumption of a macro-layer underneath vapour mushrooms, nucleate boiling is highly difficult to predict accurately. In addition, the small scales of physical mechanisms and the high impact of the heating surface on nucleate boiling make the development of heat transfer correlations even more difficult. This mainly explains why an important number of nucleate boiling correlations have been reported in the literature. For instance, while investigating the performance of their thermosyphons, Jouhara and Robinson [98] and Jafari et al. [18] compared the experimental boiling heat transfer coefficient with eight and six nucleate pool boiling correlations, respectively. Indeed, regarding the numerous correlations presented, it becomes difficult to determine which model best suits a given situation. In contrast, when numerically estimating the boiling resistance of a TPCT, many scientists (see for instance refs: [20], [97], [99]-[101]) only refer to the most well-known nucleate boiling correlation by Rohsenow [102]. It appears that many researchers using thermosyphons for industrial applications only select the most commonly used boiling correlations. In addition, the few analyses of correlations available in the literature often report the accuracy obtained by the author of the correlation himself. Often, empirical or semi-empirical correlations use adjustable constants chosen to fit the author's experiments. However, when analysed by other researchers and compared to a different set of boiling data, the accuracy of the correlation can 
vary significantly [35]. Then, little value should be granted to the accuracy reported by authors of the correlation, as the correlation was adapted to fit their own data. Instead, an analysis by external authors with different sets of boiling data should be preferred to check the accuracy and reliability of a given correlation. Despite the complexity of choosing a suitable pool boiling correlation, the accuracy of the prediction is of importance when designing a thermosyphon-based system for an industrial application. Indeed, the estimation of the TPCT performance is directly related to the system performance and therefore is linked to the economic profit made and the return-on-investment time of the heat-pipe based installation. In this regard, a critical review of nucleate pool boiling correlations seems relevant in providing thermosyphon users with an analysis of a large number of predictive models. Besides referencing a high number of correlations, the objectives of this section are to bring clarity on the use of each correlation, analyse each model based on the work reported in the literature and sum up the numerous studies in a comprehensive way. Finally, recommendations are made to provide keys and meticulously to choose a nucleate pool boiling correlation when estimating the boiling resistance of a thermosyphon.

To determine the parameters required to develop nucleate pool boiling correlations, the Buckingham-pi theorem can be used [97]. Accordingly to Newton's law (Eq. (1)), the nucleate boiling heat transfer $h_{n b}$ can mainly be described using the wall superheat $\Delta T_{s a t}$, the latent heat of vaporization $i_{l v}$, the body force implied by the liquid-vapour density difference $g\left(\rho_{l}-\rho_{v}\right)$, the surface tension $\sigma$, a characteristic length $L$, and the working fluid thermophysical properties $\rho, C_{p}, k, \mu$ as shown in Eq. (42).

$$
h_{n b}=f\left(\Delta T_{s a t}, g\left(\rho_{l}-\rho_{v}\right), i_{l v}, \sigma, L, \rho, c_{p}, k, \mu\right)
$$

In terms of dimensionless parameters, it follows that:

$$
N u=\frac{h_{n b} L}{k}=f\left[\frac{\rho g\left(\rho_{l}-\rho_{v}\right) L^{3}}{\mu^{2}}, J a=\frac{C_{p} \Delta T}{i_{l v}}, \operatorname{Pr}=\frac{\mu C_{p}}{k}, B o=\frac{g\left(\rho_{l}-\rho_{v}\right) L^{2}}{\sigma}\right]
$$

where $\mathrm{Nu}, \mathrm{Ja}, \mathrm{Pr}$ and $\mathrm{Bo}$ are the Nusselt, Jakob, Prandtl and Bond number, respectively. Yet, some factors are often modified. In particular, instead of using the wall superheat, the nucleate boiling heat flux $q_{n b}{ }_{n b}$ is widely used. Moreover, due to their demonstrated significant influence of bubbles on nucleate boiling, parameters such as the number of active nucleation sites $N_{a}$, the bubble departure diameter $D_{d}$ and the bubble departure frequency $f_{d}$ are commonly introduced. A state of the art of nucleate pool boiling correlations, sorted from most ancient to the most recent, is presented in Table 6. As it is possible for TPCTs' users to determine the heat flux $q_{n b}$ by using the electrical input of the heat source, pool boiling correlation including the heat flux as a parameter can easily be used. Therefore, and in contrast with the precedent state of the art of correlations for the bubble departure diameter and frequency, correlations using the heat flux are considered in this state of the art. 
Table 6: Nucleate pool boiling heat transfer coefficient $h_{n b}$ correlations - State of the Art

\begin{tabular}{|c|c|c|c|c|}
\hline Authors & Correlation & Specific parameter and condition & $\begin{array}{c}\text { Frequency } \\
\text { of use }\end{array}$ & Year \\
\hline $\begin{array}{l}\text { Kruzhilin } \\
{[103]}\end{array}$ & $\begin{array}{l}\quad h_{n b}=0.082\left(\frac{k_{l}}{L_{b}}\right)\left(\frac{i_{l v} q_{n b}}{g T_{s a t} k_{l}} \frac{\rho_{l}}{\rho_{l}-\rho_{v}}\right)^{0.7}\left(\frac{T_{s a t} c_{p, l} \sigma \rho_{l}}{i_{l v}{ }^{2} \rho_{v}{ }^{2} L_{b}}\right)^{0.33} P r_{l}{ }^{-0.45} \\
\text { where, } \\
* \quad L_{b}=\left[\frac{\sigma}{g\left(\rho_{l}-\rho_{v}\right)}\right]^{1 / 2}\end{array}$ & & Very low & 1947 \\
\hline $\begin{array}{l}\text { Rohsenow } \\
{[102]}\end{array}$ & $\begin{array}{l}\text { In terms of wall superheat: } \\
\qquad h_{n b}=\mu_{l} i_{l v}\left[\frac{g\left(\rho_{l}-\rho_{v}\right)}{\sigma}\right]^{1 / 2} \operatorname{Pr}_{l}^{-s / r}\left(\frac{c_{p, l}}{C_{s f} i_{l v}}\right)^{1 / r}\left(\Delta T_{s a t}\right)^{\frac{1-r}{r}} \\
\text { In terms of heat flux: } \\
\qquad h_{n b}=\left(\frac{q_{n b}^{\prime \prime}}{i_{l v}}\right)^{1-r}\left[\mu_{l} / \sqrt{\frac{\sigma}{g\left(\rho_{l}-\rho_{v}\right)}}\right]^{r} \frac{c_{p, l}}{C_{s f}} \operatorname{Pr}_{l}^{-s} \\
\text { where, } \\
* \begin{array}{c}r=1 / 3 \\
s=n=1 \text { for water } \\
s=n=1.7 \text { for other fluids }\end{array}\end{array}$ & $\begin{array}{l}\text { - } C_{s f} \text { is a constant depending on the solid- } \\
\text { fluid characteristics }\end{array}$ & Very high & 1952 \\
\hline $\begin{array}{l}\text { McNelly } \\
{[104]}\end{array}$ & $h_{n b}=0.225\left(\frac{q_{n b}{ }_{n b} c_{p}}{i_{l v}}\right)^{0.69}\left(\frac{P k_{l}}{\sigma}\right)^{0.31}\left(\frac{\rho_{l}}{\rho_{v}}-1\right)^{0.31}$ & & Very low & 1953 \\
\hline $\begin{array}{l}\text { Forster and } \\
\text { Zuber } \\
{[105]}\end{array}$ & $h_{n b}=\frac{0.00122 \times \Delta T_{s a t}{ }^{0.24} \Delta P_{s a t}{ }^{0.75} c_{p, l}{ }^{0.45} \rho_{l}{ }^{0.49} k_{l}{ }^{0.79}}{\sigma^{0.5} i_{l v}{ }^{0.24} \mu_{l}{ }^{0.29} \rho_{l}{ }^{0.24}}$ & $\begin{array}{l}\Delta P_{\text {sat }}=\left.P_{v}\right|_{T=T_{w}}-\left.P_{v}\right|_{T=T_{\text {sat }}} \text { is the } \\
\text { difference in saturation (vapour) pressure } \\
\text { between the wall temperature and the } \\
\text { saturation temperature }\end{array}$ & Medium & 1955 \\
\hline Tien [23] & $h_{n b}=61.3 k_{l} \operatorname{Pr}_{l}^{0.33} N_{a}^{0.5} \Delta T_{\text {sat }}$ & & Very low & 1962 \\
\hline $\begin{array}{l}\text { Lienhard } \\
{[106]}\end{array}$ & $h_{n b}=C k_{l} \operatorname{Pr}^{1 / 3} \frac{\left.\sqrt{\sigma g\left(\rho_{l}-\rho_{v}\right) / \rho_{l}^{2}}\right|_{\text {fluid }}}{\left.\sqrt{\sigma g\left(\rho_{l}-\rho_{v}\right) / \rho_{l}^{2}}\right|_{\text {water }}} N_{a}^{1 / 3}\left(\Delta T_{\text {sat }}\right)^{5 / 4}$ & $\begin{array}{l}\text { The studied fluid is compared to a reference } \\
\text { fluid often chosen as water }\end{array}$ & Very low & 1963 \\
\hline
\end{tabular}




\begin{tabular}{|c|c|c|c|c|}
\hline & $\begin{array}{l}\text { where, } \\
\star C \text { is am empirical constant }\end{array}$ & & & \\
\hline $\begin{array}{l}\text { Mostinskii } \\
{[107]}\end{array}$ & $\begin{array}{l}\qquad h_{n b}=3.596 \times 10^{-5} P_{c r i t}{ }^{0.69}\left(q_{n b}\right)^{0.7} F\left(P^{*}\right) \\
\text { where, } \\
* \quad F\left(P^{*}\right)=1.8 P^{* 0.17}+4 P^{* 1.2}+10 P^{* 10}\end{array}$ & $\begin{array}{l}P^{*}=P / P_{\text {crit }} \text { is the dimensionless reduced } \\
\text { pressure }\end{array}$ & Low & 1963 \\
\hline $\begin{array}{l}\text { Mikic and } \\
\text { Rohsenow } \\
{[34]}\end{array}$ & $\begin{array}{l}\qquad h_{n b}=\left[1-N_{a} K\left(\pi d_{d}^{2} / 4\right)\right] h_{n c}+h_{b} \\
\text { where, } \\
* h_{b}=2 N_{a} D_{d}^{2}\left(\pi k_{l} c_{p, l} \rho_{l} f_{d}\right)^{1 / 2} \\
* K=\frac{T_{c r i t} \ln \left(P^{*}\right)}{\left(1-T_{c r i t}\right)} \\
\text { To estimate the value of bubble related factors } N_{a}, D_{d} \text { and } f_{d} \text {, authors proposed: } \\
* \quad N_{a}=r_{s}^{m}\left(\frac{i_{l v} \rho_{v}}{2 T \sigma}\right)^{m} \Delta T_{\text {sat }} m \\
* D_{d}=a\left[\frac{\sigma}{g\left(\rho_{l}-\rho_{v}\right)}\right]^{1 / 2}\left(\frac{\rho_{l} c_{p, l} T_{s a t}}{\rho_{v} i_{l v}}\right)^{5 / 4} \\
* f_{d}=\frac{0.6}{D_{d}}\left[\frac{\sigma g\left(\rho_{l}-\rho_{v}\right)}{\rho_{v}^{2}}\right]^{1 / 4} \\
*\left\{\begin{array}{c}a=1.5 \times 10^{-4} \text { for water } \\
a=4.65 \times 10^{-4} \text { for other fluids }\end{array}\right.\end{array}$ & $\begin{array}{l}h_{n c} \text { is the natural convection heat transfer } \\
\text { coefficient occurring on the surface not } \\
\text { affected by bubbles. This must be calculated } \\
\text { regarding the geometry by using natural } \\
\text { convection correlations. } \\
\text { * } m \text { is a coefficient that can be obtained by } \\
\text { measuring the cavity radius distribution or } \\
\text { to fit experimental data } \\
\text { * Authors considered that transient heat } \\
\text { transfer occurs in a zone of diameter } 2 d_{b} \\
\text { (equivalent to } K=4 \text { ) }\end{array}$ & Low & 1969 \\
\hline $\begin{array}{l}\text { Danilova } \\
{[108]}\end{array}$ & $\begin{array}{l}\qquad h_{n b}=C\left(\frac{R_{a}}{R_{a 0}}\right)^{0.2}\left(0.14+2.2 P^{*}\right) q_{n b} \quad 0.75 \\
\text { where, } \\
* \quad C \text { is an empirical constant }\end{array}$ & * The correlation was developed for Freon & Very low & 1970 \\
\hline $\begin{array}{l}\text { Labuntsov } \\
{[109]}\end{array}$ & $h_{n b}=0.075\left[1+10\left(\frac{\rho_{v}}{\rho_{l}-\rho_{v}}\right)^{0.67}\right]\left(\frac{k_{l}^{2}}{v_{l} \sigma T_{\text {sat }}}\right)^{0.33} q_{n b}^{\prime \prime}$ & & Medium & 1973 \\
\hline $\begin{array}{l}\text { Imura et al. } \\
{[110]}\end{array}$ & $h_{n b}=0.32\left(\frac{\rho_{l}{ }^{0.65} k_{l}{ }^{0.3} c_{p, l}{ }^{0.7} g^{0.2}}{\rho_{l}{ }^{0.25} i_{l v}{ }^{0.4} \mu_{l}}\right)\left(\frac{P_{v}}{P_{a t m}}\right)^{0.3} q_{n b}{ }_{n b}^{0.4}$ & $\begin{array}{l}\text { Developed especially for thermosyphons } \\
\text { (TPCT) }\end{array}$ & Very high & 1979 \\
\hline
\end{tabular}




\begin{tabular}{|c|c|c|c|c|}
\hline $\begin{array}{l}\text { Stephan } \\
\text { and } \\
\text { Preusser } \\
{[111]}\end{array}$ & $h_{n b}=0.1\left(\frac{k_{l}}{D_{d}}\right)\left(\frac{q_{n b}{ }_{n b} D_{d}}{k_{l} T_{\text {sat }}}\right)^{0.67}\left(\frac{\rho_{v}}{\rho_{l}}\right)^{0.156}\left(\frac{i_{l v} D_{d}{ }^{2}}{\alpha_{l}{ }^{2}}\right)^{0.371}\left(\frac{\alpha_{l}^{2} \rho_{l}}{\sigma D_{d}}\right)^{0.35}\left(\frac{\mu_{l} c_{p, l}}{k_{l}}\right)^{-0.16}$ & & Very low & 1979 \\
\hline $\begin{array}{l}\text { Stephan } \\
\text { and } \\
\text { Abdelsalam } \\
{[112]}\end{array}$ & 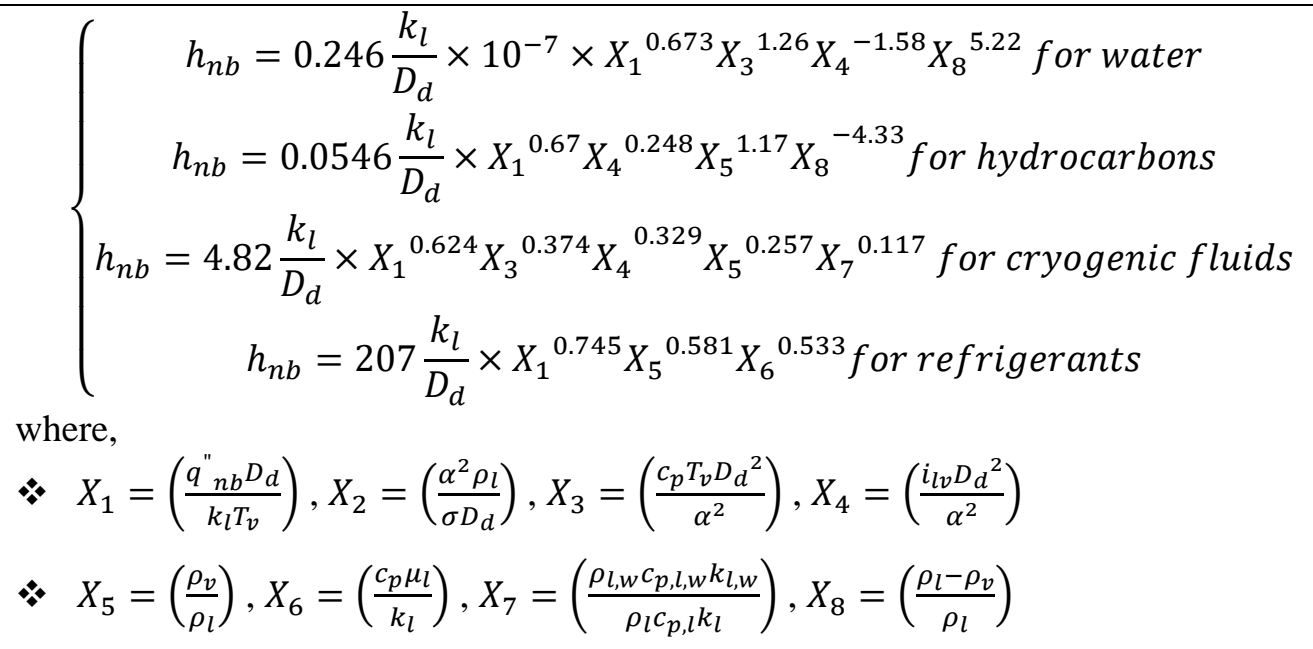 & $\begin{array}{l}10^{-4} \leq P^{*} \leq 0.9 \text { and } \theta=45^{\circ} \text { for water } \\
5.7 \times 10^{-3} \leq P^{*} \leq 0.9 \text { and } \theta=35^{\circ} \text { for } \\
\text { hydrocarbons } \\
\quad 4 \times 10^{-3} \leq P^{*} \leq 0.97 \text { and } \theta=1^{\circ} \text { for } \\
\text { cryogenic fluids } \\
\quad 3 \times 10^{-3} \leq P^{*} \leq 0.78 \text { and } \theta=35^{\circ} \text { for } \\
\text { refrigerants }\end{array}$ & High & 1980 \\
\hline $\begin{array}{l}\text { Shiraishi et } \\
\text { al. [113] }\end{array}$ & $h_{n b}=0.32\left(\frac{\rho_{l}^{0.65} k_{l}^{0.3} c_{p, l}{ }^{0.7} g^{0.2}}{\rho_{l}^{0.25} i_{l v}^{0.4} \mu_{l}^{0.1}}\right)\left(\frac{P_{v}}{P_{a t m}}\right)^{0.23} q_{n b}{ }^{0.4}$ & $\begin{array}{l}\text { Developed especially for thermosyphons } \\
\text { (TPCTs) }\end{array}$ & High & 1981 \\
\hline Bier [114] & $\begin{array}{l}\qquad h_{n b}=3.596 \times 10^{-5} P_{c r i t}{ }^{0.69}\left(q_{n b}^{\prime \prime}\right)^{0.7} F\left(P^{*}\right) \\
\text { where, } \\
* \quad F\left(P^{*}\right)=0.7+2 P^{*}\left(4+\frac{1}{1-P^{*}}\right)\end{array}$ & $\begin{array}{l}P^{*}=P / P_{\text {crit }} \text { is the dimensionless reduced } \\
\text { pressure }\end{array}$ & Very low & 1982 \\
\hline $\begin{array}{l}\text { Nishikawa } \\
{[115]}\end{array}$ & $\begin{array}{l}\quad h_{n b}=12.7\left(\frac{p_{\text {crit }}{ }^{2} R_{\text {mol }} T_{\text {crit }}}{M_{\text {mol }}}\right)^{1 / 10} \times F_{N}\left(P^{*}\right) \times q_{n b}^{\prime \prime}{ }_{n b}^{4 / 5} \times\left(8 R_{p} / R_{p 0}\right)^{\left(1-P^{*}\right) / 5} \\
\text { where, } \\
* \quad F_{N}\left(P^{*}\right)=P^{* 0.23}\left(1-0.99 P^{*}\right)^{0.9}\end{array}$ & $\begin{array}{l}\text { * } R_{p} \text { is the Maximum peak height of the } \\
\text { profile } \\
\text { The Maximum peak height reference } R_{p 0} \\
\text { should be taken as: } R_{p 0}=0.1 \times 10^{-6} \mathrm{~m} \\
\text { * Developed for refrigerants }\end{array}$ & Medium & 1982 \\
\hline
\end{tabular}




\begin{tabular}{|c|c|c|c|c|}
\hline & & $\begin{array}{l}P^{*}=P / P_{\text {crit }} \text { is the dimensionless reduced } \\
\text { pressure }\end{array}$ & & \\
\hline $\begin{array}{l}\text { Cooper } \\
{[116]}\end{array}$ & $h_{n b}=55\left(q_{n b}^{\prime \prime}\right)^{0.67} P^{*\left(0.12-0.2 \log R_{a, p}\right)}\left(-\log P^{*}\right)^{-0.55} M_{m o l}{ }^{-1 / 2}$ & $\begin{array}{l}R_{a, p} \text { is an average roughness parameter } \\
\text { expressed in } \mu \mathrm{m} \text {. If unknown, a default } \\
\text { value of } R_{a, p}=1 \mu \mathrm{m} \text { should be taken. }\end{array}$ & High & 1984 \\
\hline $\begin{array}{l}\text { Ueda et al. } \\
\text { [117] }\end{array}$ & $\begin{array}{l}\qquad h_{n b}=C_{s f}{ }^{-1} P r_{l}{ }^{-1.7}\left(\frac{c_{p, l}}{i_{l v}} q_{n b}{ }_{n b}\right)\left(\frac{L_{b}}{i_{l v} \mu_{l}} q_{n b}\right)^{0.7} \\
\text { where, } \\
\text { * } L_{b}=\left[\frac{\sigma}{g\left(\rho_{l}-\rho_{v}\right)}\right]^{1 / 2}\end{array}$ & $\begin{array}{l}* C_{s f} \text { is a constant depending on the solid- } \\
\text { fluid characteristics }\end{array}$ & Low & 1988 \\
\hline $\begin{array}{l}\text { Kutateladze } \\
{[118]}\end{array}$ & $\begin{array}{l}\qquad h_{n b}=0.44 P r_{l}^{0.35}\left(\frac{k_{l}}{L_{b}}\right)\left(\frac{\rho_{l}}{\rho_{l}-\rho_{v}} \frac{P \times 10^{-4}}{\rho_{v} g i_{l v} \mu_{l}} q_{n b}\right)^{0.7} \\
\text { where, } \\
\text { * } L_{b}=\left[\frac{\sigma}{g\left(\rho_{l}-\rho_{v}\right)}\right]^{1 / 2}\end{array}$ & & High & 1990 \\
\hline $\operatorname{Gro} \beta[119]$ & $h_{n b}=55 q_{n b}{ }^{0.7}\left[P^{* 0.12} /\left(\left(-\log _{10} P^{*}\right)^{0.55} \sqrt{M_{m o l}}\right)\right]$ & $\begin{array}{l}P^{*}=P / P_{\text {crit }} \text { is the dimensionless reduced } \\
\text { pressure }\end{array}$ & Very low & 1990 \\
\hline $\begin{array}{l}\text { Gorenflo et } \\
\text { al.[120] }\end{array}$ & $\begin{array}{l}h_{n b}=h_{0} F\left(P^{*}\right)\left(q_{n b}^{\prime \prime} / q_{0}\right)^{n}\left(R_{a} / R_{a 0}\right)^{0.133} \\
\text { where, } \\
*\left\{\begin{array}{c}F\left(P^{*}\right)=1.73 P^{* 0.27}+\left(6.1+\frac{0.68}{1-P^{*}}\right) P^{* 2} \text { for water } \\
F\left(P^{*}\right)=1.2 P^{* 0.27}+2.5 P^{*}+\frac{P^{*}}{1-P^{*}} \text { for all other fluids except liquid helium }\end{array}\right. \\
*\left\{\begin{array}{c}n=0.9-0.3 P^{* 0.3} \text { for water } \\
n=0.9-0.3 P^{* 0.15} \text { for all other fluids except liquid helium }\end{array}\right.\end{array}$ & $\begin{array}{l}* P^{*}=P / P_{\text {crit }} \text { is the dimensionless reduced } \\
\text { pressure } \\
\text { The standard conditions in which } h_{o} \text { must be } \\
\text { evaluated are: } P^{*}=0.1, R_{a 0}=0.4 \times \\
10^{-6} \mathrm{~m}, q_{0}{ }^{\prime \prime}=20,000 \mathrm{~W} \cdot \mathrm{m}^{-2}\end{array}$ & Medium & 1990 \\
\hline $\begin{array}{l}\text { Kaminaga } \\
\text { et al. [121] }\end{array}$ & $\begin{array}{l}\quad h_{n b}=22\left(\rho_{v} / \rho_{l}\right)^{0.4} R_{a, p}{ }^{\left(1-P^{*}\right) / 5} h_{n b, \text { Kutateladze }} \\
\text { where, } \\
* h_{n b, \text { Kutateladze }}=0.44 P r_{l}^{0.35}\left(\frac{k_{l}}{L_{b}}\right)\left(\frac{\rho_{l}}{\rho_{l}-\rho_{v}} \frac{P \times 10^{-4}}{\rho_{v} i_{l v} \mu_{l}} q_{n b}^{\prime \prime}\right)^{0.7}\end{array}$ & $\begin{array}{l}R_{a, p} \text { is an average roughness parameter } \\
\text { expressed in } \mu m \text {. }\end{array}$ & Very low & 1992 \\
\hline
\end{tabular}




\begin{tabular}{|c|c|c|c|c|}
\hline $\begin{array}{l}\text { Leiner } \\
{[122],} \\
\text { Leiner and } \\
\text { Gorenflo } \\
{[123]}\end{array}$ & 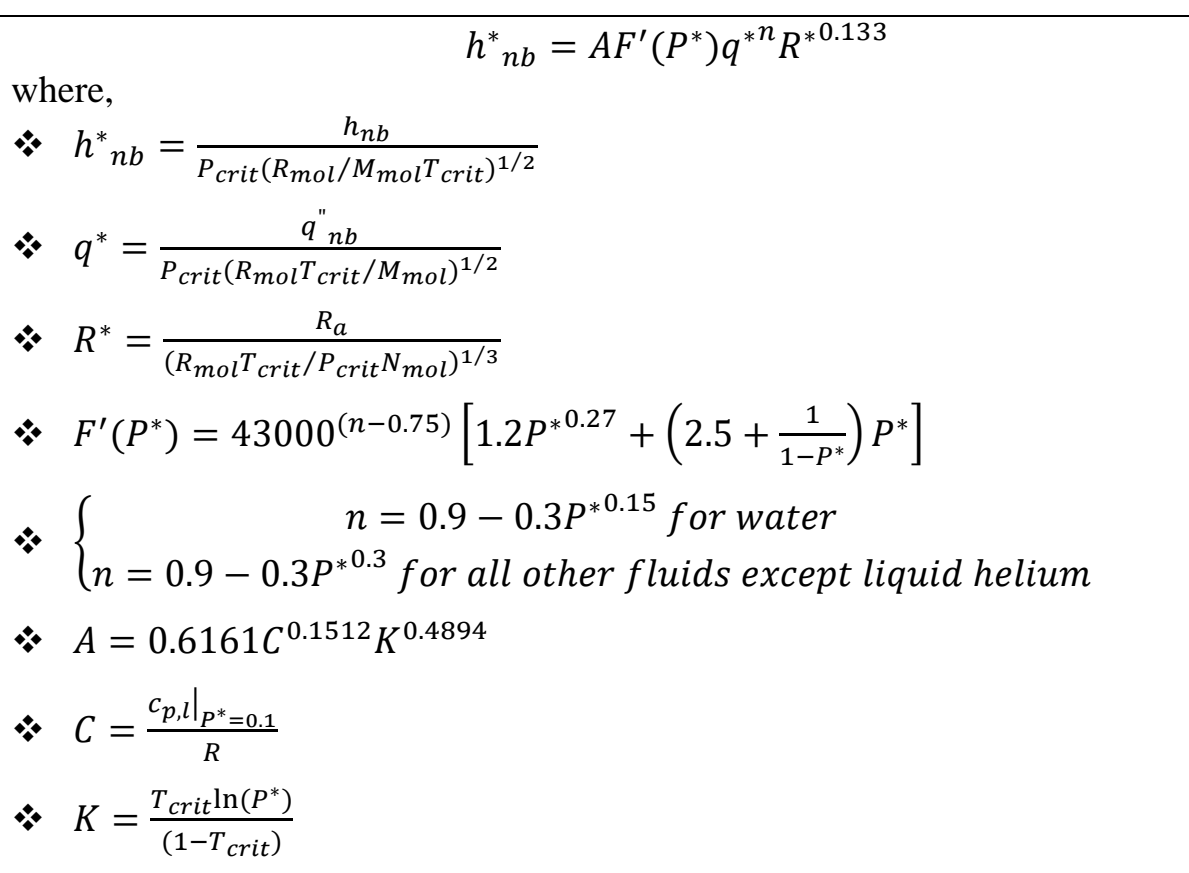 & $\begin{array}{l}\text { H } h^{*}, P^{*} \text { and } R^{*} \text { are the dimensionless } \\
\text { parameters for the heat transfer coefficient, } \\
\text { reduced pressure, heat flux and average } \\
\text { surface roughness parameter, respectively. } \\
\left.* \quad c_{p, l}\right|_{P_{r}=0.1} \text { is the molar specific heat capacity } \\
\text { at } P^{*}=0.1 \text { expressed in }\left[\mathrm{J} \cdot \mathrm{kmol}^{-1} \cdot \mathrm{K}^{-1}\right] \\
* \quad \text { If unknown, a default value } R_{a}=0.4 \times \\
10^{-6} \mathrm{~m} \text { is recommended. }\end{array}$ & Medium & 1994 \\
\hline $\begin{array}{l}\text { Chowdhury } \\
\text { et al. [124] }\end{array}$ & $\begin{array}{l}\qquad\left\{\begin{array}{l}h_{n b}=11.43\left(R e_{b}\right)^{0.72}\left(P r_{l}\right)^{0.42}\left(\frac{\rho_{v}}{\rho_{l}}\right)^{0.5}\left(\frac{d_{b}}{D_{i}}\right)\left(\frac{k_{l}}{D_{d}}\right) \text { for water } \\
h_{n b}=495.7\left(R e_{b}\right)^{0.8}\left(\operatorname{Pr}_{l}\right)^{0.5}\left(\frac{\rho_{v}}{\rho_{l}}\right)^{0.33}\left(\frac{k_{l}}{D_{d}}\right) \text { for ethanol } \\
h_{n b}=6\left(R e_{b}\right)^{0.78}\left(\operatorname{Pr}_{l}\right)^{0.48}\left(\frac{\rho_{v}}{\rho_{l}}\right)^{0.58}\left(\frac{k_{l}}{D_{d}}\right) \text { for Freon } R-113\end{array}\right. \\
\text { where, } \\
* R e_{b}=\frac{q_{n b}^{\prime \prime} D_{d}}{\rho_{v} i_{l v} v_{l}}\end{array}$ & $\begin{array}{l}\text { Developed especially for thermosyphons } \\
\text { (TPCTs) }\end{array}$ & Medium & 1997 \\
\hline $\begin{array}{l}\text { El-Genk } \\
\text { and Saber } \\
{[19]}\end{array}$ & $\begin{array}{l}h_{n b}=(1+4.95 \psi) \times h_{n b, \text { Kutateladze }} \\
\text { where, } \\
* h_{n b, \text { Kutateladze }}=0.44 P r_{l}^{0.35}\left(\frac{k_{l}}{L_{b}}\right)\left(\frac{\rho_{l}}{\rho_{l}-\rho_{v}} \frac{P \times 10^{-4}}{\rho_{v} g i_{l v} \mu_{l}} q_{n b}^{\prime \prime}\right)^{0.7}\end{array}$ & $\begin{array}{l}\text { The mixing coefficient } \psi \text { is used to describe } \\
\text { the mixing phenomenon between the bubble } \\
\text { rising along the wall and the bubble } \\
\text { generated in the pool. }\end{array}$ & Medium & 1998 \\
\hline
\end{tabular}




\begin{tabular}{|c|c|c|c|c|}
\hline & $\begin{array}{l}* \psi=\left(\frac{\rho_{l}}{\rho_{v}}\right)^{0.4}\left[\frac{P_{v} v_{l}}{\sigma}\left(\frac{\rho_{l}{ }^{2}}{\sigma g\left(\rho_{l}-\rho_{v}\right)}\right)^{1 / 4}\right]^{1 / 4} \\
* L_{b}=\left[\frac{\sigma}{g\left(\rho_{l}-\rho_{v}\right)}\right]^{1 / 2}\end{array}$ & & & \\
\hline $\begin{array}{l}\text { Kiatsiriroat } \\
\text { et al. [125] }\end{array}$ & 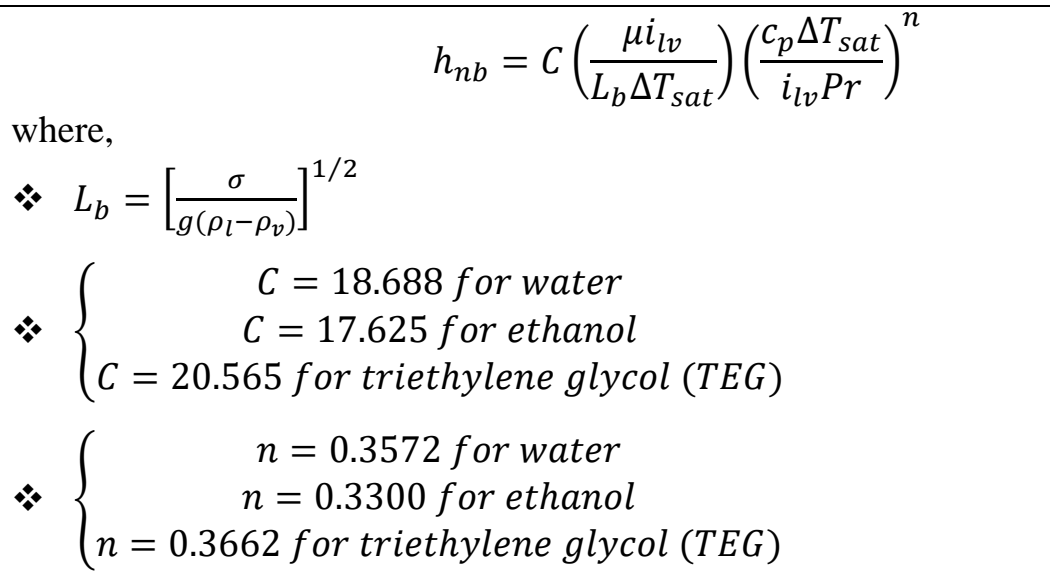 & $\begin{array}{l}\text { * Developed especially for thermosyphons } \\
\text { (TPCTs) }\end{array}$ & Very low & 2000 \\
\hline $\begin{array}{l}\text { Ribatski } \\
\text { and } \\
\text { Jabardo } \\
{[126]}\end{array}$ & $\begin{array}{l}\qquad h_{n b}=C R_{a}^{0.2} P^{* 0.45}\left[-\log \left(P^{*}\right)\right]^{-0.8} M^{-0.5} q_{n b}{ }_{n b}^{n} \\
\text { where, } \\
* \quad C \text { is an empirical constant }\end{array}$ & $\begin{array}{l}* P^{*}=P / P_{\text {crit }} \text { is the dimensionless reduced } \\
\text { pressure }\end{array}$ & Very low & 2003 \\
\hline
\end{tabular}


Several works analysing boiling correlation accuracy can be found in the literature. In a review on bubble parameters, Mohanty [35] reported seven pool boiling correlations using parameters related to bubbles such as the bubble departure diameter and frequency. The author observed that even if some correlations might fit certain boiling data, the prediction accuracy can be significantly lower for other experiments. In a global review of thermosyphons, Jafari et al. [18] compared the experimental boiling performances of a TPCT using water as a working fluid with six nucleate boiling correlations. In their experiments, authors showed the accuracy of a correlation could differ regarding the thermosyphon's filling ratio. Jouhara and Robinson [98] experimentally focused on small diameter TPCTs charged with water, FC-84, FC-77 and FC-3283 and compared the experimental evaporator resistance with eight predictive models. Pioro et al. [127] accurately analysed six existing boiling heat transfer correlations using an important database for Water, Ethanol, R-113, and n-Heptane. The Rohsenow [102] correlation was found to be the more accurate. Using a copper TPCT with FC-72 as a working fluid, Park et al.[128] investigated the influence of the filling ratio on the boiling heat transfer and compared the experimental results to the most known correlations of Rohsenow [102] and Imura et al. [110]. The predictive model from Rohsenow [102] described best the experiments. Similarly, Noie [129] compared the two models of Rohsenow [102] and Imura et al. [110] while investigating the impact of the input heat flux, filling ratio and evaporator length on a vertical copper two-phase closed thermosyphon filled with water. Even if the agreement between the models and the experiment was described as "reasonable" by the author, the correlation by Rohsenow [102] seems to present a better accuracy according to the plots. Guo and Nutter [130] compared the boiling heat transfer of two TPCTs charged with R134a with the correlation by Imura et al. [110] and found an accuracy of about 30\%. In a review on thermosyphons, Jafari et al. [131] compared five correlations with water, ethanol and R113 experimental data. According to the experiments made on water, at low heat flux, the correlation of Imura et al. [110] and Rohsenow [102] were quite accurate. In contrast, the correlations by Kutateladze [118] and Chowdhury et al. [124] seem to over predict the heat transfer. For ethanol, both Rohsenow [102] and Imura et al. [110] predictions are within an error lower than 15\%. The correlation by Stephan and Abdelsalam [112] was found really accurate. In opposition, the correlations by Kutateladze [118] and Chowdhury et al. [124] are not recommended because they over predict the heat transfer coefficient. Chowdhury and Fumito [132] experimentally investigated pool boiling of Freon R-113 in a vertical small diameter tube. The correlations of Stephan and Abdelsalam [112] and McNelly [104] predicted the experimental values within an error of $\pm 20 \%$. By compiling 731 experimental data points for various fluids, El-Genk and Saber [19] developed a new pool boiling correlation. In their work, the accuracy of the correlations by Gro $\beta$ [119], Imura et al. [110], Shiraishi et al. [113], Ueda et al. [117] and Kaminaga et al. [121] were studied. Kiatsiriroat [125] used an equation derived from the Rohsenow [102] correlation while investigating the performance of a thermosyphon heat pipe charged with TEG-water and Ethanol-water mixtures. The Rohsenow [102] correlation was able to predict most of the mixture data within $15 \%$ of error. In an experiment on a nitrogen TPCT, Nakano et al. [133] used the correlation proposed by ElGenk and Saber [19] but the accuracy of the correlation wasn't clearly analysed. Jafari et al. [134] recently studied the transient characteristics of a TPCT and showed that the Imura et al. [110] correlation is highly accurate for high filling ratios. However, the discrepancy between prediction and experiment increases with lower filling ratios.

An analysis of nucleate pool boiling correlations has been made based on the previously introduced work. This study gathers the opinions and tends to bring out the most suitable correlations while evaluating the boiling resistance of a thermosyphon in the case where only pool boiling takes place ( $F R=100 \%)$. This analysis is presented in Table 7. It must be noted that the accuracy reported by authors on their own correlation is not considered while investigating its reliability. This is considered to be of little value compared with checking the accuracy of a correlation on different sets of boiling data. 
Table 7: Nucleate pool boiling heat transfer coefficient $h_{n b}$ correlations - Analysis

\begin{tabular}{|c|c|c|c|c|}
\hline Authors & Positive points & Negative points & $\begin{array}{l}\text { Frequency } \\
\text { of use }\end{array}$ & Year \\
\hline $\begin{array}{l}\text { Kruzhilin } \\
{[103]}\end{array}$ & * Developed to suit a wide variety of fluids [98] & $\begin{array}{l}\text { More general at the expense of the accuracy [98] } \\
\text { Average mean error of } 16 \%, 23 \%, 30 \% \text { and } 19 \% \text { for Water, } \\
\text { Ethanol, R-113 and n-Heptane, respectively [127] }\end{array}$ & Very low & 1947 \\
\hline $\begin{array}{l}\text { Rohsenow } \\
{[102]}\end{array}$ & $\begin{array}{l}* \text { The fluid-surface interface in considered by the coefficient } C_{s f} \\
\text { that gives flexibility and possible adjustments of the correlation } \\
* \quad \text { Adaptable for a wide range of surface material/fluid } \\
\text { combinations } \\
* \quad \text { Good at low heat fluxes too } \\
* \quad \text { Independent of the heater geometry and orientation [18] } \\
* \quad \text { Accurate for small diameter TPCTs [98] } \\
* \quad \text { Average mean error of } 2 \%, 4 \%, 3 \% \text { and } 1 \% \text { for Water, Ethanol, } \\
\text { R-113 and n-Heptane, respectively [127] } \\
* \quad \text { Good agreement despite a slight over prediction at higher heat } \\
\text { fluxes [18] } \\
* \quad \text { Better prediction than Imura et al. [128], [129] } \\
* \quad \text { Error lower than } 15 \% \text { [125] }\end{array}$ & $\begin{array}{l}\text { * The value of the coefficient } C_{s f} \text { required to characterize the } \\
\text { surface-fluid interface can be difficult to estimate accurately } \\
* \quad \text { Slight over prediction at filling ratio }<100 \%[18] \\
* \quad \text { Less accurate for very small filling ratio }( \pm 30 \%)[18]\end{array}$ & Very high & 1952 \\
\hline $\begin{array}{l}\text { McNelly } \\
{[104]}\end{array}$ & * Accuracy about $\pm 20 \%$ for R-113 [132] & $\begin{array}{l}\text { Accuracy } \pm 20 \%- \pm 40 \% \text { for water, benzene and Isopropanol } \\
\text { [35] }\end{array}$ & Very low & 1953 \\
\hline $\begin{array}{l}\text { Forster and } \\
\text { Zuber } \\
{[105]}\end{array}$ & & * The surface-fluid interface is not considered & Medium & 1955 \\
\hline Tien [23] & & $\begin{array}{l}\text { The density of active nucleation sites is often unknown and } \\
\text { experimental values are taken } \\
* \quad \text { The constant } 61.3 \text { was obtained from water boiling only }\end{array}$ & Very low & 1962 \\
\hline
\end{tabular}




\begin{tabular}{|c|c|c|c|c|}
\hline & & $\begin{array}{l}\text { The number of active nucleation site exponents may vary in the } \\
\text { range } N_{a}^{0.3}-N_{a}^{0.5} \text {, depending on the fluid considered, which } \\
\text { seems inaccurate }\end{array}$ & & \\
\hline $\begin{array}{l}\text { Lienhard } \\
{[106]}\end{array}$ & * Expression derived from Tien [23] correlation & $\begin{array}{l}\text { The studied fluid is compared to a reference fluid which is } \\
\text { laborious } \\
\text { * The empirical constant } C \text { is actually used to fit experimental } \\
\text { data }\end{array}$ & Very low & 1963 \\
\hline $\begin{array}{l}\text { Mostinskii } \\
{[107]}\end{array}$ & * Number of physical properties reduced by using pressure & & Low & 1963 \\
\hline $\begin{array}{l}\text { Mikic and } \\
\text { Rohsenow } \\
\text { [34] }\end{array}$ & & $\begin{array}{l}\text { * This correlation based on the bubble growth model from a } \\
\text { surface requires parameters related to the bubble formation } \\
\left(N_{a}, d_{d}, f\right) \text { that are difficult to obtain. Many other correlations } \\
\text { than those proposed by the authors are reported in the literature } \\
\text { to evaluate these coefficients } \\
\text { * This equation also requires natural convection correlations } \\
\text { * The value of coefficient } m \text { is not accurate } \\
\text { * The proposed equation neglects the latent heat transport and the } \\
\text { temperature gradient into the fluid }\end{array}$ & Low & 1969 \\
\hline $\begin{array}{l}\text { Danilova } \\
\text { [108] }\end{array}$ & & $\begin{array}{l}\text { The empirical constant } C \text { is actually used to fit experimental } \\
\text { data } \\
* \quad \text { The correlation was developed for Freon }\end{array}$ & Very low & 1970 \\
\hline $\begin{array}{l}\text { Labuntsov } \\
{[109]}\end{array}$ & $\begin{array}{l}* \text { Developed to suit a wide variety of fluids [98] } \\
\quad \text { Accurate for small diameter TPCTs [98] }\end{array}$ & $\begin{array}{l}\text { More general at the expense of the accuracy [98] } \\
\text { Important under-prediction. Less accurate for small filling } \\
\text { ratios. Not recommended [18] } \\
\text { Average mean error of } 24 \%, 25 \%, 26 \% \text { and } 7 \% \text { for Water, } \\
\text { Ethanol, R-113 and n-Heptane, respectively [127] }\end{array}$ & Medium & 1973 \\
\hline $\begin{array}{l}\text { Imura et al. } \\
{[110]}\end{array}$ & $\begin{array}{l}\text { Covers all the regimes from natural convection to fully } \\
\text { developed nucleate boiling [18], [19] }\end{array}$ & $\begin{array}{ll}* & \text { Slight under prediction at filling ratio }<100 \% \text { [18] } \\
* & \text { Over prediction higher than } 30 \% \text { for small diameter TPCTs [98] }\end{array}$ & Very high & 1979 \\
\hline
\end{tabular}




\begin{tabular}{|c|c|c|c|c|}
\hline & $\begin{array}{l}\text { The correlation was found accurate by the following } \\
\text { references: [128]-[130] } \\
\text { * Excellent accuracy at high filling ratio FR }=100 \% \text { [134] } \\
\text { Good agreement at low and high heat flux }( \pm 10 \%) \text { despite a } \\
\text { slight under prediction at higher heat fluxes for small filling } \\
\text { ratios. Stays quite accurate regardless of the filling ratio [18] } \\
\text { * Developed especially for pool boiling in thermosyphons } \\
\text { (TPCTs) }\end{array}$ & $\begin{array}{ll}* & \text { Accuracy about 30\% for a TPCT charged with R134a [130] } \\
* & \text { Large discrepancy with the data by Kaminaga et al. [121][19] } \\
* & \text { Not advised for small filling ratio [134] }\end{array}$ & & \\
\hline $\begin{array}{l}\text { Stephan } \\
\text { and } \\
\text { Preusser } \\
{[111]}\end{array}$ & & $\begin{array}{l}\text { * Empirical using bubble parameters } \\
\text { * Accuracy } \pm 20 \%- \pm 50 \%[35]\end{array}$ & Very low & 1979 \\
\hline $\begin{array}{l}\text { Stephan } \\
\text { and } \\
\text { Abdelsalam } \\
{[112]}\end{array}$ & $\begin{array}{l}\text { Made by regression analysis methods over } 5000 \text { existing } \\
\text { experimental data points } \\
* \text { Applicable for different fluids } \\
* \text { Accurate for small diameter TPCTs [98] } \\
\text { * Accuracy } \pm 10 \%- \pm 20 \% \text { for four sets of data (water, Benzene } \\
\text { and Isopropanol), predicted all data with a reasonable accuracy } \\
\text { [35] } \\
* \text { Accuracy lower than } \pm 20 \% \text { for R-113 [132] }\end{array}$ & $\begin{array}{l}\text { * Initially made for natural convection boiling but often used in } \\
\text { nucleate boiling too } \\
* \quad \text { Accuracy } \pm 40 \%- \pm 50 \% \text { for two sets of data (water) [35] }\end{array}$ & High & 1980 \\
\hline $\begin{array}{l}\text { Shiraishi et } \\
\text { al. [113] }\end{array}$ & $\begin{array}{l}\text { Derived from Imura et al. [110] by changing the coefficient } 0.3 \\
\text { to } 0.23 \\
* \text { Developed especially for thermosyphons (TPCTs) } \\
* \text { Accuracy estimated about } \pm 30 \% \text { at low heat flux and about } \\
\pm 10 \% \text { at high heat flux }[19]\end{array}$ & $\begin{array}{l}\text { - Less accurate than Imura et al. [110] } \\
\text { * Slight under prediction[18]. } \\
\text { The correlation seems to be sensitive to the operating } \\
\text { * Large discrepancy with the data by Kaminaga et al. [121][19] }\end{array}$ & High & 1981 \\
\hline Bier [114] & $\begin{array}{ll}* & \text { Expression derived from Mostinskii [107] correlation } \\
* & \text { Number of physical properties reduced by using pressure }\end{array}$ & & Very low & 1982 \\
\hline
\end{tabular}




\begin{tabular}{|c|c|c|c|c|}
\hline $\begin{array}{l}\text { Nishikawa } \\
\text { [115] }\end{array}$ & * The surface roughness is considered [74] & $\begin{array}{l}\text { The absence of fluid-specific parameters suggests this equation } \\
\text { is generally suitable only for the fluids examined by the authors. } \\
\text { Modifications are needed for this correlation to suit other fluids }\end{array}$ & Medium & 1982 \\
\hline $\begin{array}{l}\text { Cooper } \\
\text { [116] }\end{array}$ & $\begin{array}{l}\text { The expression comes from an analysis of a wide range of } \\
\text { boiling data } \\
\text { The surface roughness is considered }\end{array}$ & $\begin{array}{l}\text { The generalization in terms of the square root of the molecular } \\
\text { weight } M_{m o l} \text { is oversimplified and can bring significant errors } \\
\text { [21] } \\
\text { * Not accurate for } P^{*}=P / P_{\text {crit }} \leq 0.08 \\
\text { The surface wettability isn't considered } \\
\text { For water, the mean absolute error ranged from } 44 \% \text { to } 324 \% \text {. } \\
\text { Not accurate for low reduced pressure }\left(P^{*}=P / P_{\text {crit }} \leq 0.08\right) \\
\text { [135] }\end{array}$ & High & 1984 \\
\hline $\begin{array}{l}\text { Ueda et al. } \\
\text { [117] }\end{array}$ & $\begin{array}{l}\text { Derived from the Rohsenow [102] correlation to fit } \\
\text { experimental data } \\
* \text { The fluid-surface interface in considered by the coefficient } C_{s f}\end{array}$ & $\begin{array}{l}\text { The values of } C_{s f} \text { measured experimentally vary from } \\
\text { Rohsenow [102] } \\
* \text { Accuracy about } 30 \%[19]\end{array}$ & Low & 1988 \\
\hline $\begin{array}{l}\text { Kutateladze } \\
\text { [118] }\end{array}$ & * Developed to suit a wide variety of fluids [18], [98] & $\begin{array}{l}\text { More general at the expense of the accuracy [18], [98] } \\
\text { Important under-prediction, less accurate for small filling ratio } \\
\text { [18] } \\
\text { Under prediction higher than } 30 \% \text { for small diameter TPCTs } \\
\text { [98] } \\
\text { Average mean error of } 22 \%, 40 \%, 47 \% \text { and } 13 \% \text { for Water, } \\
\text { Ethanol, R-113 and n-Heptane, respectively [127] }\end{array}$ & High & 1990 \\
\hline Gro $\beta$ [119] & $\begin{array}{l}\text { * Derived from Cooper correlation } \\
\text { * Based on } 2529 \text { experimental data }\end{array}$ & $\begin{array}{l}\text { The generalization in terms of the square root of the molecular } \\
\text { weight } M_{m o l} \text { is oversimplified and can bring significant errors } \\
\text { [21] } \\
* \quad \text { Reported accuracy about } 30 \%[19] \\
\text { * Observed accuracy about } 60 \%[19]\end{array}$ & Very low & 1990 \\
\hline $\begin{array}{l}\text { Gorenflo et } \\
\text { al.[120] }\end{array}$ & $\begin{array}{l}\text { Values of } h_{0} \text { have been reported for numerous fluids in the } \\
\text { literature (for water, } h_{0}=5600 \text { ) }\end{array}$ & $\begin{array}{l}\text { The main limit is that the heat transfer coefficient } h_{n b} \text { is related } \\
\text { to a standard one } h_{o} \text { that must be evaluated accurately }\end{array}$ & Medium & 1990 \\
\hline
\end{tabular}




\begin{tabular}{|c|c|c|c|c|}
\hline & $\begin{array}{l}\text { * If the value of } h_{0} \text { is not reported, Gorenflo et al.[120] } \\
\text { recommend to use the Stephan and Preusser [111] correlation } \\
\text { (as cited in Ref. [136]) } \\
\text { * The surface roughness is considered } \\
\text { * } \text { Nice accuracy for low surface roughness } \\
\text { * Accommended for water } \\
\text { The correlation is well-describing small surface roughness heat } \\
\text { transfer coefficients [135] }\end{array}$ & & & \\
\hline $\begin{array}{l}\text { Kaminaga } \\
\text { et al. [121] }\end{array}$ & $\begin{array}{l}* \text { Derived from Kutateladze correlation } \\
* \quad \text { Suitable for small pool }(D \leq 37 \mathrm{~mm})\end{array}$ & $\begin{array}{l}\text { Over-prediction at low heat flux [19] } \\
\text { Reported accuracy for water, ethanol and R-113 about } \pm 20 \% \text {, } \\
\pm 20 \% \text { and } \pm 30 \% \text {, respectively [19] } \\
\text { * Observed accuracy of } \pm 40 \% \text { [19] }\end{array}$ & Very low & 1992 \\
\hline $\begin{array}{l}\text { Leiner } \\
{[122],} \\
\text { Leiner and } \\
\text { Gorenflo } \\
{[123]}\end{array}$ & $\begin{array}{l}\text { This correlation is recommended for poorly known fluids } \\
\text { * Dimensionless form of the Gorenflo et al.[120] correlation } \\
\text { * No more standard value required } \\
\text { Other values of coefficient } A \text { are also reported. In particular, } \\
\text { factors considering gravity and surface tension can slightly } \\
\text { improve the accuracy of } A\end{array}$ & $\begin{array}{l}\text { * This correlation is less accurate than the Gorenflo et al.[120] } \\
\text { correlation: The RMS-deviation is about } 14 \% \\
\text { * This equation is not recommended for water (in this case, the } \\
\text { Gorenflo et al.[120] equation should be preferred) } \\
\text { * Not accurate for water (mean absolute error about 50\%) [135] }\end{array}$ & Medium & 1994 \\
\hline $\begin{array}{l}\text { Chowdhury } \\
\text { et al. [124] }\end{array}$ & $\begin{array}{l}\text { * Developed especially for thermosyphons (TPCTs) } \\
\text { * Developed for water, ethanol and R113 } \\
\text { * This correlation is suitable for confined regions where the } \\
\text { bubble diameter is comparable with the tube diameter [98] } \\
\text { * More accurate than Kutateladze and Labuntsov [18] }\end{array}$ & $\begin{array}{l}\text { * Important under-prediction. Less accurate for small filling ratio. } \\
\text { Not recommended [18]. } \\
\text { * Accuracy about } 15 \% \text { [98] }\end{array}$ & Medium & 1997 \\
\hline $\begin{array}{l}\text { El-Genk } \\
\text { and Saber } \\
{[19]}\end{array}$ & * Equation derived from Kutateladze correlation [118] & & Medium & 1998 \\
\hline
\end{tabular}




\begin{tabular}{|c|c|c|c|c|}
\hline & $\begin{array}{l}\text { The mixing coefficient } \psi \text { is used to describe the mixing } \\
\text { phenomenon between the bubble rising along the wall and the } \\
\text { bubble generated in the pool. } \\
\text { * Developed to suit various fluids such as water, ethanol, } \\
\text { methanol, Dowtherm-A, R-11 and R-113 } \\
\text { * Suitable for small pool }(D \leq 37 \mathrm{~mm}) \\
\text { * Small over prediction [18] } \\
\text { * Accuracy of } \pm 15 \%[131]\end{array}$ & & & \\
\hline $\begin{array}{l}\text { Kiatsiriroat } \\
\text { et al. [125] }\end{array}$ & $\begin{array}{l}* \text { Derived from Rohsenow correlation } \\
* \text { Can be used for } \mathrm{FR}<100 \% \text { as it considers falling film } \\
\text { evaporation }\end{array}$ & The values of the empirical constant $C$ can be discussed & Very low & 2000 \\
\hline $\begin{array}{l}\text { Ribatski } \\
\text { and } \\
\text { Jabardo } \\
{[126]}\end{array}$ & & $\begin{array}{l}\text { The generalization in terms of the square root of the molecular } \\
\text { weight } M_{m o l} \text { is oversimplified and can bring significant errors } \\
\text { [21] } \\
\text { * The empirical constant } C \text { is actually used to fit experimental } \\
\text { data }\end{array}$ & Very low & 2003 \\
\hline
\end{tabular}


In the analysis presented, it can be observed that the most known correlations are also the most analysed. Therefore, a good idea of the reliability of these models is reported in the literature. In comparison, many models are only listed but little investigated by external authors. In particular, the use of recent correlations remains hazardous, as their reliability has not been tested on a larger scale yet. Nevertheless, it comes out that the most known correlations are often the more accurate or are correlations that represent a breakthrough in the knowledge of nucleate pool boiling heat transfer. Based on the references [18], [19], [35], [98], [125], [127]-[132], [134], in order to estimate the nucleate pool boiling heat transfer coefficient in a thermosyphon where only pool boiling occurs ( $F R=100 \%)$, the following recommendations are made: Regardless of its popularity, the first predictive model advised is the Rohsenow [102] correlation. Indeed, despite its wide use, its accuracy, reliability and advantages in comparison to other available correlations was proved. It comes out that the correlation from Rohsenow [102] shows a constant reliability and a good accuracy in most of the nucleate pool boiling situations. The use of a coefficient $C_{s f}$ to characterize the surface-fluid interaction is relevant as it allows a flexibility regarding the material and fluid used. Moreover, thermosyphon users who need a quick estimate of the boiling heat transfer can rely on the $C_{s f}$ table reported in the literature whereas the most meticulous researchers can estimate the value of a coefficient $C_{s f}$ by considering the micro-geometry of the surface, the nucleation and bubble growth process and the surface-liquid small-scale interactions, among others. One must be aware that the accuracy of the correlation by Rohsenow [102] decreases with the filling ratio which seems to show this correlation is suitable for pool boiling only in the case where $\mathrm{FR}=100 \%$. The second correlation recommended was developed by Imura et al. [110]. This correlation was especially developed for thermosyphons and shows an excellent accuracy at high filling ratios. In some cases, though, this correlation showed inaccuracy. For instance, this correlation is not advised in the case of small diameter TPCTs. Hence, the use of this correlation must be made carefully and should only be applied when only pool boiling takes place at the thermosyphon's evaporator. Then, the correlation by Stephan and Abdelsalam [112] is strongly recommended. As this correlation was built using regression analysis methods over 5000 existing experimental data points, the chances that this correlation suits the boiling heat transfer of a given TPCT are high. This predictive model also shows a good flexibility as it can be used for various fluids such as water, Benzene, Isopropanol and R113. Moreover, this correlation seems to be quite insensitive to the thermosyphon diameter. Finally, the recent correlation by El-Genk and Saber [19] seems promising. Again, the correlation was developed using a large data base which is relevant regarding the high discrepancy between boiling experiments. The correlation is suitable for water, ethanol, methanol, Dowtherm-A, R-11 and R-113 regardless of the pool diameter. Finally, the bubble mechanism is introduced by using a mixing coefficient $\psi$ that may be modified in the future to describe better the participation of bubbles in nucleate boiling. Yet, due to its relatively recent release, proving the reliability of this correlation by a higher number of authors would be wise. A conclusion, summing up the analysis of the most suitable nucleate pool boiling correlations, when estimating the boiling heat transfer coefficient of a thermosyphon, is presented in Table 8.

Table 8: Nucleate pool boiling heat transfer coefficient $h_{n b}$ correlations - Recommendations

\begin{tabular}{|c|c|c|c|c|}
\hline Authors & $\begin{array}{c}\text { Frequency } \\
\text { of use }\end{array}$ & Year & Comments & $\begin{array}{l}\text { Recomm } \\
\text { endation }\end{array}$ \\
\hline $\begin{array}{l}\text { Rohsenow } \\
\text { [102] }\end{array}$ & Very high & 1952 & $\begin{array}{l}\text { Widely used and tested } \\
\text { * Good accuracy and reliability for most nucleate pool } \\
\text { boiling } \\
\text { * Adaptable for a wide range of surface material/fluid } \\
\text { combinations by adjusting the value of the coefficient } \\
C_{s f} \\
\text { * The coefficient } C_{s f} \text { also allows the consideration of } \\
\text { the micro-geometry and the nucleation process } \\
\text { Less accurate for very small filling ratio }\end{array}$ & 1 \\
\hline
\end{tabular}




\begin{tabular}{|c|c|c|c|c|c|}
\hline $\begin{array}{l}\text { Imura et al. } \\
\text { [110] }\end{array}$ & Very high & 1979 & $*$ & $\begin{array}{l}\text { Widely used and tested } \\
\text { Covers all the regimes from natural convection to fully } \\
\text { developed nucleate boiling } \\
\text { Excellent accuracy at high filling ratio FR=100\% in } \\
\text { the case where pool boiling only is taking place } \\
\text { Developed especially for pool boiling in } \\
\text { thermosyphons (TPCTs) } \\
\text { Not advised for small diameter TPCTs ( } D \leq 37 \mathrm{~mm} \text { ) }\end{array}$ & 2 \\
\hline $\begin{array}{l}\text { Stephan and } \\
\text { Abdelsalam } \\
\text { [112] }\end{array}$ & High & 1980 & * & $\begin{array}{l}\text { Made by regression analysis methods over } 5000 \\
\text { existing experimental data points } \\
\text { Accuracy estimated about } \pm 10 \%- \pm 20 \% \\
\text { Correlation suitable and accurate for a high proportion } \\
\text { of pool boiling experiments } \\
\text { Applicable for different fluids such as water, Benzene, } \\
\text { Isopropanol and R-113 } \\
\text { Stays accurate for small diameter TPCTs ( } D \leq \\
37 \mathrm{~mm} \text { ) }\end{array}$ & 3 \\
\hline $\begin{array}{l}\text { El-Genk and } \\
\text { Saber [19] }\end{array}$ & Medium & 1998 & $*$ & $\begin{array}{l}\text { Good accuracy despite possible small over prediction } \\
\text { Based on a wide data source of } 731 \text { boiling points } \\
\text { Developed to suit various fluids such as water, } \\
\text { ethanol, methanol, Dowtherm-A, R-11 and R-113 } \\
\text { Suitable for small pools ( } D \leq 37 \mathrm{~mm} \text { ) } \\
\text { The bubble dynamic is considered using the mixing } \\
\text { coefficient } \psi \\
\text { Recent correlation: reliability to be proved by more } \\
\text { authors }\end{array}$ & 4 \\
\hline
\end{tabular}

It should be noticed that the presented models to calculate the nucleate pool boiling heat transfer coefficient are often two-dimensional models. Therefore, the material and surface characteristics, in addition to the heat-flux and temperature distribution in the wall, are supposed to be constant and homogeneous in the thermosyphon section and all along the evaporator. Then, a discrepancy could be observed between the experiments and the theoretical models as the thermal characteristics of a TPCT are not perfectly uniform in all the directions. Nevertheless, according to Rohsenow [21], the simplification may be reasonable for relatively thin walls.

While investigating nucleate pool boiling, it quickly emerges that a high discrepancy between the experiments is observed [19], [126]. This main obstacle is still faced by researchers who struggle to develop accurate models to predict boiling heat transfer. To overcome this problem, scientists are following two separate paths. On the one hand, with the help of more and more powerful equipment, many are investigating in detail the micro-mechanisms occurring in nucleate pool boiling, such as the nucleation process, bubble growth, wall temperature fluctuation, microlayer evaporation, sensible heat transport, latent heat transport and macro-layer phenomena to improve the accuracy of detailed but laborious models. With the same effort, some scientists are investigating an isolated factor on nucleate pool boiling such as the surface aspect, material, number of active nucleation sites, wall thickness, pressure, subcooling, presence of dissolved gases, gravity, hysteresis or inclination angle among others. On the other hand, while confronted with the highly scattered nucleate boiling data, scientists try to develop more user-friendly correlations to fit most of the boiling cases. So far, it has been shown that, in the case of TPCT users, these correlations are widely used. Indeed, in the applications of 
thermosyphons, the boiling resistance remains a source of uncertainties when predicting the performance of an installation.

\section{FURTHER INVESTIGATIONS ON NUCLEATE POOL BOILING}

\subsection{Pool boiling in small diameter pipes}

For some applications such as compact heat exchangers [98], [137], small-diameter thermosyphons can be used. Due to the confined space, the boiling mechanisms are changing and the pool boiling heat transfer correlations used to estimate the performances of a small diameter thermosyphon must be adapted. To describe the confinement and narrow passage in small tubes, the confinement number describing the importance of the bubble length scale $L_{b}$ to the internal diameter of the pipe $D_{i}$ is introduced [137], [138]:

$$
C o=\frac{L_{b}}{D_{i}}=\frac{\left[\sigma / g\left(\rho_{l}-\rho_{v}\right)\right]^{1 / 2}}{D_{i}}
$$

With $L_{b}$ the bubble length scale $(\mathrm{m})$, and $D_{i}$ the internal diameter of the pipe (m). According to references [138]-[141], the effect of confinement on boiling should be addressed for tubes with a confinement number $0.5<\mathrm{Co}$. Indeed, when the bubble diameter becomes significant in comparison with the TPCT diameter, the bubble growth is governed by the narrow boiling space. In confined spaces, it was showed that a decrease in the tube diameter leads to higher bubble rise velocity [141]. Furthermore, bubbles can fill the narrow tube section and, in that case, are separated from the wall by a liquid layer that evaporates, thus, generating an exponential and rapid growth of the bubble [137], [141]. This mechanism is similar to the microlayer evaporation underneath the base of a bubble. The thin film evaporation between the bubble and the wall tends to enhance the boiling heat transfer. However, under the action of the vapour going up, liquid entrainment is more likely to take place and a slug flow can appear. In addition, partial dry-out is eased due to the important volume of a bubble compared to that of the pool. Ishibashi and Nishikawa [141] attest that beside the typical isolated bubble region of pool boiling, a coalesced bubble region exists in tube with restricted sections. In the isolated bubble region in narrow tubes, it seems that saturated boiling is similar to the common pool boiling. However, in the coalesced bubble region, merged bubbles are growing and rise regularly at a low departure frequency [141]. Then, the boiling characteristics in the coalesced bubble region are significantly different and so is the boiling heat transfer. In their study, Ishibashi and Nishikawa [141] observed that the coalesced bubble region stands for tubes with a boiling space dimension $\Delta R$ lower than $3 \mathrm{~mm}$. By investigated boiling of distilled water, sodium oleate aqueous solution, saponin aqueous solution, and ethyl alcohol, Ishibashi and Nishikawa [141] obtained the evolution of the boiling heat transfer coefficient with the boiling space dimension $\Delta R$ showed in Fig. 18. 

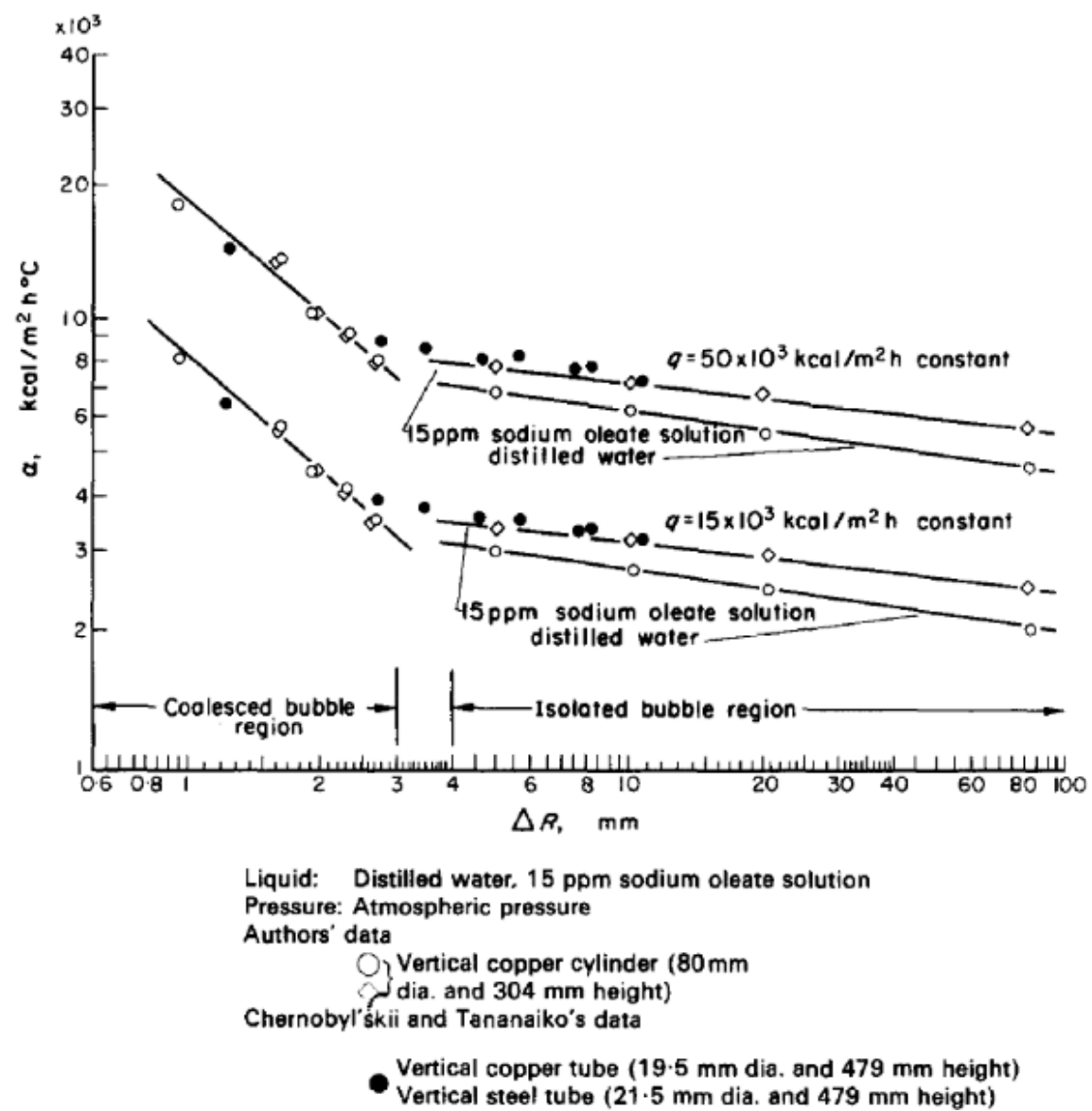

Figure 18: Variation of heat-transfer coefficients $(\alpha)$ due to change of boiling space dimensions, from Ishibashi and Nishikawa [141]

According to the study of Ishibashi and Nishikawa [141] presented in Fig. 18, the pool boiling heat transfer coefficient is higher in the coalesced bubble region. For a space dimension of $\Delta R=0.97 \mathrm{~mm}$, the boiling heat transfer coefficient in the coalesced bubble region was almost 4 times higher than common pool boiling in larger tubes. Ishibashi and Nishikawa [141] also proposed correlations to describe the influence of the narrow space on pool boiling heat transfer coefficient including one equation for the coalesced bubble region in the form:

$$
h_{n b, \text { confined }}=\frac{k_{l}}{\Delta R} 13.6 \times\left(R e^{*}\right)^{2 / 3}
$$

with,

$$
\left\{\begin{array}{c}
R e^{*}=\frac{q^{\prime \prime}}{i_{l v} \rho_{v}} \frac{\Delta R}{v_{l}}\left(\frac{q^{\prime \prime}}{q_{0}{ }^{\prime \prime}}\right) \\
q_{0}{ }^{\prime \prime}=1.6 \times 10^{6} \mathrm{~W} \cdot \mathrm{m}^{-2}
\end{array}\right.
$$

where $k_{l}$ is the liquid thermal conductivity $\left(\mathrm{W} \cdot \mathrm{m}^{-1} \cdot \mathrm{K}^{-1}\right), \Delta R$ the boiling space dimension (m), $q^{\prime \prime}$ the boiling heat flux per unit surface area $\left(\mathrm{W} . \mathrm{m}^{-2}\right), i_{l v}$ the latent heat of vaporization $\left(\mathrm{J} \cdot \mathrm{kg}^{-1}\right)$, and $v_{l}$ the kinematic viscosity of the liquid $\left(\mathrm{m}^{2} \cdot \mathrm{s}^{-1}\right)$. Similarly to Ishibashi and Nishikawa [141], Yao and Chang [142] identified three boiling mechanisms in narrow tubes: isolated bubbles, slightly deformed bubbles, and coalesced deformed bubbles. Yao and Chang [142] investigated pool boiling in a confined space for Freon-113, acetone and water at $1 \mathrm{~atm}$ for annuli with gap sizes of 0.32, 0.80 and $2.58 \mathrm{~mm}$. Fujita et al. [143] investigated nucleate pool boiling of saturated water at atmospheric pressure in narrow spaces between rectangular surfaces with a gap in the range 5-0.15 mm. At a gap of $5 \mathrm{~mm}$, the boiling regime was similar to unconfined pool boiling. At moderate gaps between $2 \mathrm{~mm}$ to $0.6 \mathrm{~mm}$, isolated bubbles were deformed, flattened and grow rapidly. In addition of the rapid grow of bubbles, it was 
assumed by Fujita et al. [143] that the evaporation of the liquid film between the bubble and the wall also enhances significantly the boiling heat transfer. At a gap of $0.15 \mathrm{~mm}$, the heating surface was completely covered by a vapour bubble formed by the merging of smaller bubbles. Local dry-out was also observed. Fujita et al. [143] compared their experiment to the heat transfer correlation for confined space by Ishibashi and Nishikawa [141] (Eq. 49) and concluded that the predictive model was in fairly good agreement (accuracy $\pm 30 \%$ ). To evaluate the boiling heat transfer coefficient in small diameter thermosyphons, Jouhara and Robinson [98] compared their experimental values with the correlation by Chowdhury et al. [124] presented in Table 6 that takes into account the confinement factor. The agreement was reasonable with an over prediction of the heat transfer coefficient about $15 \%$. Smith et al. [138] studied the impact of confinement in a fully transparent thermosyphon using water, ethanol and HFE-7000. It was found that the reduce pressure inside the TPCT has an influence on the bubble size, what is of high importance in the case of confined space. Indeed, at low reduced pressure, the bubble can be large enough to occupy the whole tube section, authors attest. In the study by Kew and Cornwell [137] boiling in a single, small-diameter tube with diameters in the range $1.39 \leq D \leq 3.69$ $\mathrm{mm}$ was investigated. The fluids used were R141b and Flutec PP1. In this work, the motion of the liquid was forced by external means and thus, flow boiling was studied. Nevertheless, authors compared their experimental data for flow boiling in a small diameter tube to both flow boiling and nucleate poolboiling heat transfer correlations. The authors [137] found that the flow boiling correlation described reasonably well the heat transfer for the largest tube diameters studied but failed into predicting the boiling heat transfer for small diameters. Instead, the nucleate pool boiling correlation by Cooper [116] best described the boiling data in small tubes and is advised. It was concluded by Kew and Cornwell [137] that nucleate pool boiling correlation can be used for flow boiling in small diameter tubes with a confinement number $C o<0.3$. A similar conclusion was made by Lazarek and Black [144] who studied boiling of R113 in a $3.15 \mathrm{~mm}$ diameter tube, which corresponds to a confinement number $C o=0.35$. They observed that in the case of narrow tubes, the mass flux and flow quality had little influence on boiling that mainly depended on the heat flux. Hence, it seems that flow boiling in small diameter tubes can be described by nucleate pool boiling correlations.

\subsection{Geyser boiling}

Under some conditions, a large bubble with a diameter in the scale of the tube diameter can be formed in the pool at the evaporator of a thermosyphon. In that case, liquid entrainment is likely to occur and the large bubble expels a significant portion of the liquid trapped above the vapour bubble to the condenser: this is called geyser boiling. The geysering mechanism in a pool is schematized in Fig. 19 [145].

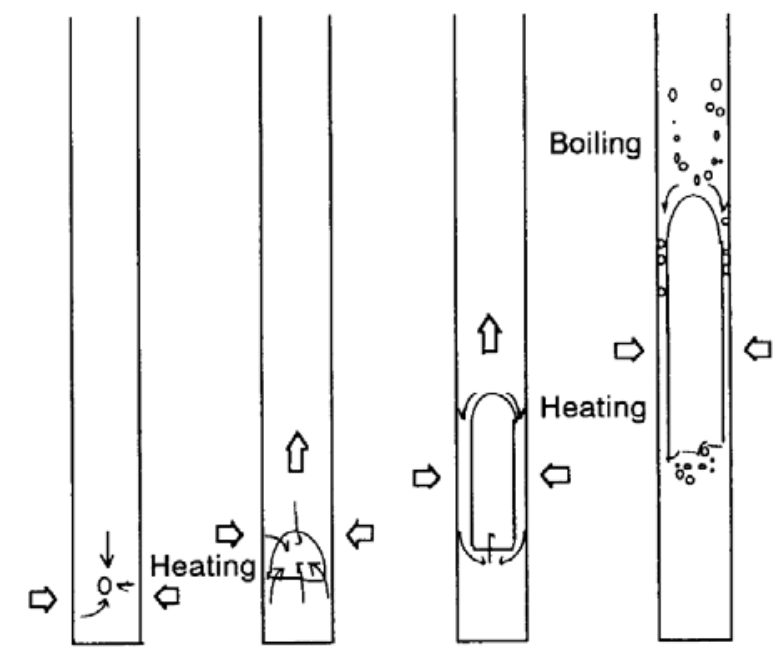

Figure 19: Geyser boiling in a pool, from Kuncoro et al. [145] 
In geyser boiling, a periodic entrainment of a portion of liquid by a large vapour bubble generate a shock in the condenser. In addition to the violent vibrations observed, a typical sound can be heard due to the explosive collision of the large liquid mass entrained to the condenser [146], [147]. After the projection of a portion of liquid, the working fluid returns to the evaporator by gravity. It was found that, in geyser boiling, the pressure rise projecting the liquid mass flow from the pool to the condenser is much shorter (milliseconds) than the time needed for the flow to return to more stable conditions [148]. The rapid growth of a large bubble that propel liquid to the condenser is to avoid in thermosyphons and can generate important vibrations and high pressure pics in the thermosyphon [147]-[150]. Even if geysering is reported to slightly impact the performances of a thermosyphon, it is mostly studied as it is a source of a mechanical issues and can lead to shock damages in thermosyphons [148].

To bring light on the mechanisms leading to geyser boiling, Morgan and Brandy [149] focus on the bubble dynamic. According to the authors, [149], the formation of a large vapour portion can be explained by the coalescence of smaller bubbles, moving from a high static pressure region to a lower static pressure region. It was observed that a bubble situated in the wake of another travels faster than the above bubble and tends to overtake it. This may also favour the coalescence of smaller bubbles into a larger vapour portion that could lead to geyser boiling. Yet, another bubble mechanism was reported by Lin et al. [151] who observed the quick growth of a single bubble emerging from the most active nucleation site in the pool, until it reaches a size comparable to the tube diameter. The relevance of this mechanism as a source of geyser boiling was later confirmed by visual experimentation by Wang et al. [152], as attest their observation presented in Fig. 20.

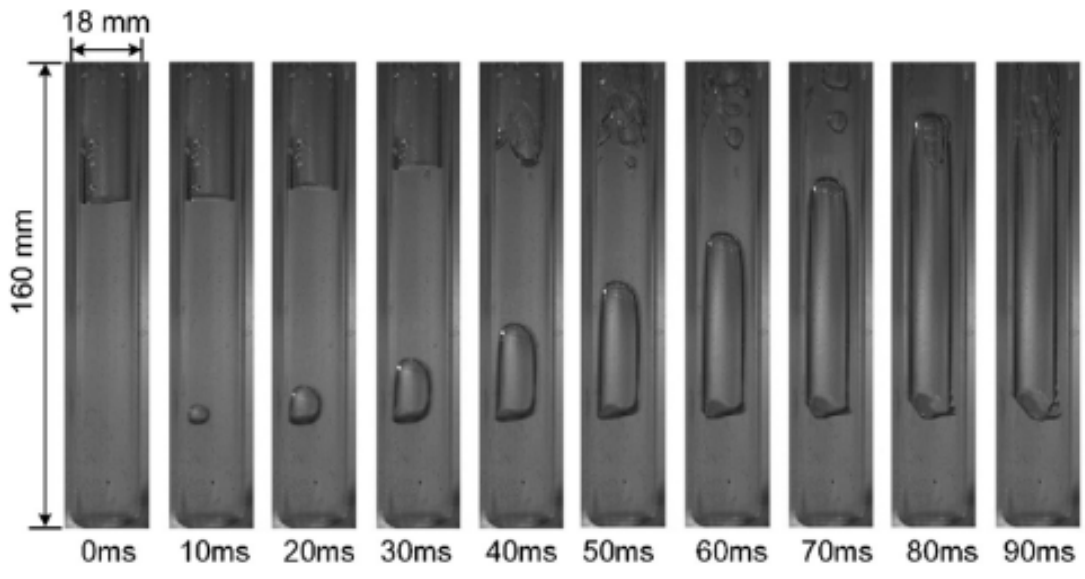

Figure 20: Growth of a single bubble into a high diameter vapour portion, from $W$ ang et al. [152]

In Fig. 20, it is clear that a single bubble grows and reaches a significant volume what begets geyser boiling. According to the literature [153], the phenomena of geyser boiling takes place at low heat flux and is eased in the case of smooth surface where the number of active nucleation sites is low. In addition, as geysering is characterize by the ratio of bubble diameter to the inside tube diameter, geyser boiling is more likely to occur in confined thermosyphon as visually showed in the work by Smith et al. [154]. In their study, Smith et al. [154] attest that during geyser boiling, heat is conveyed both by latent and sensible heat transport.

Many researchers have investigated the impact of several factors on geyser boiling. Based on the references [145], [146], [148], [151], the reported factors impacting geysering in the pool of a thermosyphons are: internal diameter of the TPCT, internal pressure, filling ratio, working fluid used, length of the evaporator section, temperature in the condenser, input heat flux, inclination angle, and thermophysical properties of the thermosyphon. Emami et al. [146] investigated the effect of factors such as inclination angle, filling ratio, input heat flux, coolant mass flow rate, and inside diameter of 
the tube on geyser boiling of distilled water. It was found that decreasing the inclination angle decreases the period of temperature oscillation in the TPCT wall and reduces the intensity of the geyser boiling impact. It was also observed that a higher filling ratio leads to stronger impact inside the thermosyphon. However, according to their study, there is no effect of the coolant mass flow on geyser boiling. At high heat flux, authors [146] attest geyser boiling didn't take place due to high pressure inside the thermosyphon preventing the formation of a large bubble in the scale of the tube diameter. Finally, it was showed that smaller thermosyphon diameters favour the geyser mechanism. Lin et al. [151] studied the effect of the heat flux, condenser temperature, filling ratio and evaporator's length on geyser boiling. It was observed that, with higher heat fluxes, shorter evaporator's length, and smaller filling ratio, geyser appear more frequently. For lower filling ratios of $F R=50 \%$ and $F R=30 \%$, no geyser were observed. Indeed, and in agreement with the references [134], [145], [151], [155], geyser boiling is more likely to appear at high filling ratios. In the case of a copper inclined thermosyphon using water and ethanol with an internal diameter $D_{i}=13 \mathrm{~mm}$, Negishi and Sawada [147] observed that geyser boiling occurred at a filling ratio $F R$ higher than $70 \%$. Hence, they advised to use filling ratios lower than $60 \%$. However, there is little chance for this criterion to be applied to a wider scale as this limit is likely to depend on the internal diameter of the TPCT, of the working fluid used and surface material chosen. The influence of the filling ratio, aspect ratio, heat input and coolant mass flow on geyser boiling in a thermosyphon was also focused by Khazaee et al. [155]. Authors found that the frequency of geyser increased with an increase of heat flux and aspect ratio and a decrease in the filling ratio. Xia et al. [156] studied the instabilities in a flat thermosyphon using water, acetone and ethanol. They report that geyser boiling can be source of mechanical damage and can threaten the safe operation of a thermosyphon. It was found that the filling ratio had an effect on the boiling instabilities only at low and medium heat flux. At higher heat flux, the instabilities are govern by the heat input and the fluctuations are decreasing. An interesting fact highlighted by the work of Xia et al. [156] is that falling droplet can generate an homogeneous nucleation process and form a bubble inside the pool. According to Kuncoro et al. [145], the initiation of a geyser is strongly impacted by the temperature distribution in the thermosyphon. Thus, the authors [145] deduced that geysering is also affected by the geometry and thermal properties of the TPCT. Recently, Kujawska et al. [157] investigated the influence of the thermosyphon's working fluid on geyser boiling. The working fluid tested were pure deionised-water, deionised-water with sodium dodecyl (surfactant), a silica nanofluid, and a nanofluid with nanohorn nanoparticles. They concluded that geysering is strongly affected by the working fluid, in particular, by the presence of chemical stabilisers. In the special case of nanoparticles, the solid-vapour interface and thus geysering is affected.

By the variation of pressure generated inside the thermosyphon by geysering, the boiling heat transfer coefficient may be impacted. Indeed, as reported by Lee and Mital [158], the heat transfer in thermosyphon is quite sensitive to the operating pressure. Yet, according to Noie et al. [150] and Jafari et al. [134], geysering does not affect the heat transfer performances. Hence, it seems that geysering only has a small impact on the pool boiling heat transfer coefficient. The most known reported heat transfer correlation for geysering in a pool was made by Casarosa [159] who correlated the pool boiling heat transfer coefficient for geyser boiling in the form:

$$
h_{n b, \text { geyser }}=2.925 P_{v}^{0.18} q^{2 / 3}
$$

where $P_{v}$ is the vapour pressure in bar, and $q^{\prime \prime}$ the heat flux per unit surface area $\left(\mathrm{W} \cdot \mathrm{m}^{-2}\right)$. The accuracy of the correlation is estimated around $20 \%$ and was also recommended by Lin et al. [151] to describe the heat transfer coefficient for geysering in pool boiling.

Geyser boiling was also studied computationally. In 2016, Jouhara et al. [153] developed the first model of geyser boiling using computational fluid dynamic (CFD) method. To do so, a mass source term was added to the continuity equation in the volume of fluid (VOF) model to consider the mass 
transition during the phase change. A preview of the CFD geyser boiling model developed by Jouhara et al. [153] is presented in Fig. 21.

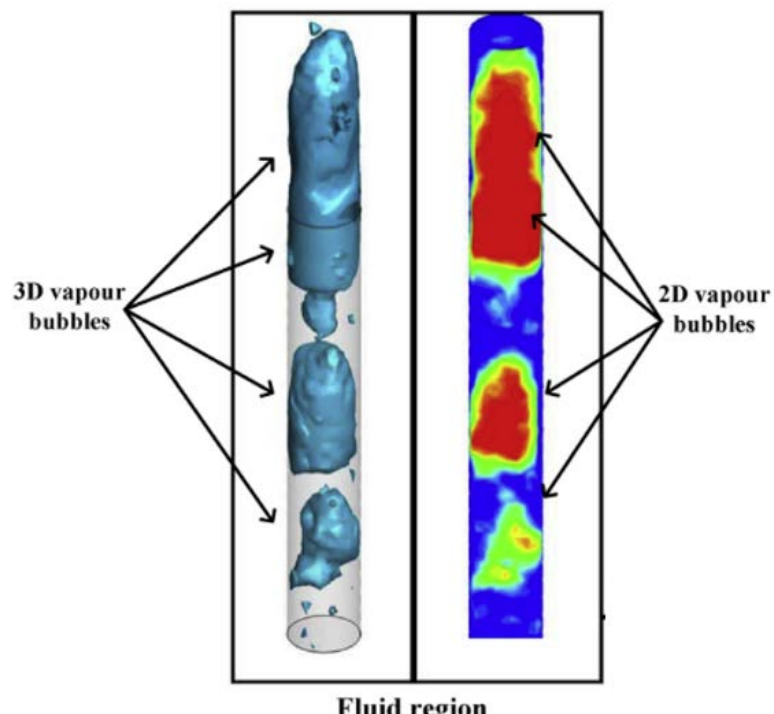

Figure 21: CFD modelling of geyser, from Joubara et al. [153]

To attest the reliability of the model, the CFD results have been compared to visual experimentation of geyser boiling in a glass thermosyphon and validated by the authors [153]. To model geyser boiling, Wang et al. [152] used the volume of fluid method including an improved Lee model to consider the superheat. According to a comparison with visual experiments, it was concluded that the improved Lee model introduced has a better prediction performance than the original model.

\subsection{Pool boiling modelling and simulations}

As briefly presented along this work, with the advances of numerical models and increase of computational power, computational fluid dynamic (CFD) simulations are opening a new area in the study of pool boiling heat transfer. This can be highlighted by the preceding investigation of geyser boiling using simulations by Jouhara et al. [153] and Wang et al. [152]. In the objective of making the reader aware of the potential of this new technique to investigate pool boiling, several works are reviewed hereafter.

In order to bring light on the bubble mechanisms implied in pool boiling, Stephan and Hammer [160] first used computational simulations to show the isotherms distribution around a bubble. It was demonstrated with the simulation that the heat flow is mainly concentrated in the micro region and that about $38 \%$ of the total heat flow goes through this region. Dhir [74] is one of the first researcher who associated both mathematical model and simulations to study bubbles in pool boiling. Based on the simulation, Dhir [74] investigated the impact of the contact angle on the bubble growth and estimated that the evaporation of the macrolayer represent about $20 \%$ of the bubble growth contribution. Low gravity condition could also be investigated. Based on the simulations, new boiling heat transfer correlations were reported. Stephan and Fuchs [77] developed a fully transient numerical model to investigate the local heat distribution in the heater wall during boiling. The computational model was used to predict the heat and fluid flows during the entire growth cycle of a bubble from a single nucleation site. Using the simulation data, it was showed that the heating surface decreases in temperature during the bubble growth stage. In addition, it was concluded that the capability of the wall of temporarily storing energy should be considered in the development of boiling correlations. Nam et al. [65] investigated the bubble departure diameter in water and developed a balance model between the buoyancy force and the surface tension. A preview of their work is shown in Fig. 22. 


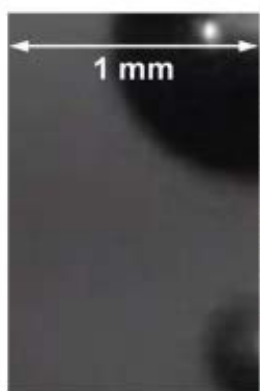

(a) $\mathrm{t}_{1}{ }^{\circ}=0.042$

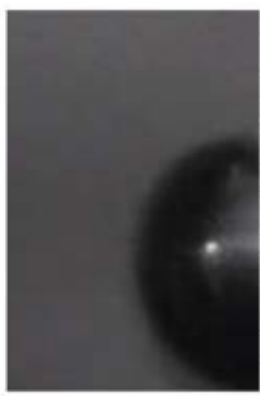

(c) $\mathrm{t}_{1}{ }^{*}=0.271$

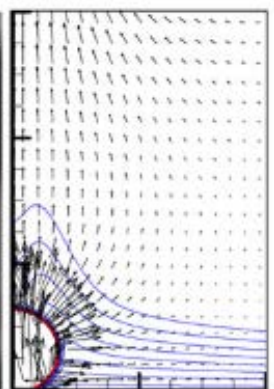

$\mathrm{t}_{2}{ }^{*}=0.046$

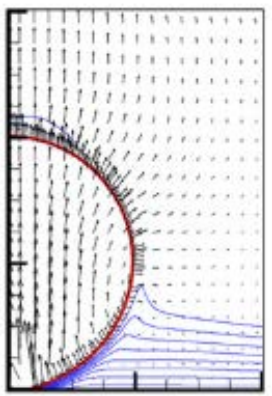

$\mathrm{t}_{2}{ }^{*}=0.266$

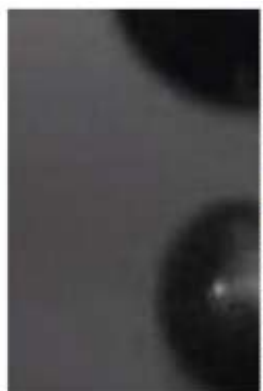

(b) $\mathrm{t}_{1}{ }^{\circ}=0.146$

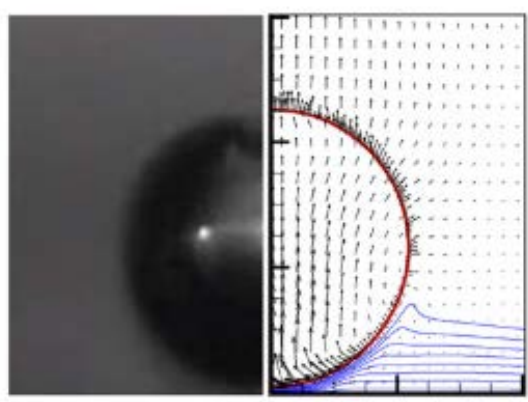

(d) $t_{1}{ }^{*}=0.334$

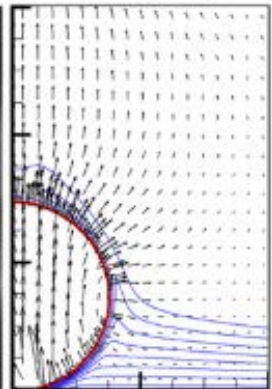

$\mathrm{t}_{2}{ }^{*}=0.146$

$\mathrm{t}_{2}{ }^{*}=0.366$

Figure 22: Simulated bubble shapes, temperature distributions, and velocity vectors, from Nam et al. [65]

The simulation agreed relatively well with the experimental observations. The study numerical and experimental study by Nam et al. [65] provided knowledge on the development of micro/nano surfaces for enhanced boiling.

To develop a boiling simulation of a complete pool, modelling technics have been developed. The most common and widely used method is known as the Volume Of Fluid (VOF) method. This method was used by De Schepper et al. [161], [162] who showed that boiling can be modelled using the Volume Of Fluid (VOF) model using a Piecewise Linear Interface Calculation (PLIC) method for reconstruction of the interface between the two-phase. The evaporation model by De Schepper et al. [161], [162] is commonly used develop user defined functions (UDF) and describe the mass transfer occurring during boiling. To dispense with the demanding interface reconstruction between the two phases that requires a high computational effort, Brackbill et al. [163] developed a new method for modelling surface tension effect in a two-phase flow, known as the continuum surface force (CSF) model. This method is often used to model boiling as it is reported to eliminate the need for interface reconstruction and allow an accurate modelling of the two-phases for lower computational expense.

Using these methods, efforts have been made by researchers to model a complete pool boiling. Yet, before 2010, many reported models such as the works by Legierski et al. [164] or Zang et al. [165] have successfully modelled evaporation and condensation but failed into simulating boiling and the apparition of bubbles in the pool. Later, Alizadehdakhel et al. [166] presented a two-dimensional twophase flow model of a thermosyphon and simulated boiling and condensation phenomena using the volume of fluid (VOF) technique. Authors [166] included the volume fraction to the source terms proposed by De Schepper et al. [161]. At the bottom of the evaporator, the simulation showed bubbles forming a vapour layer near the wall and then moving to the centre of the thermosyphon at the top of the evaporator. The agreement between numerical predictions and experimental results for the temperature distribution was good for the evaporator and condenser but lower for the adiabatic section as heat losses are not considered in the model. The simulations by Annamalai and Ramalingam [167] and Lin et al. [168] have highlighted the capability of CFD modelling to predict the temperature distribution during pool boiling in a thermosyphon. Fadhl et al. [169] simulated boiling in a thermosyphon with ANSYS FLUENT using the VOF method and a UDF including the mass source 
term of De Schepper et al. [161] to take into account the mass transition between the two-phases. The continuum surface force (CSF) model by Brackbill et al. [163] was implemented for the interfacial surface tension. In addition, the mixture properties of the two-phase flow were calculated for each computational cell by the model using a barycentre made with the volume fraction of each phase. A preview of the pool boiling simulation in a thermosyphon by Fadhl et al. [169] is presented in Fig. 23.

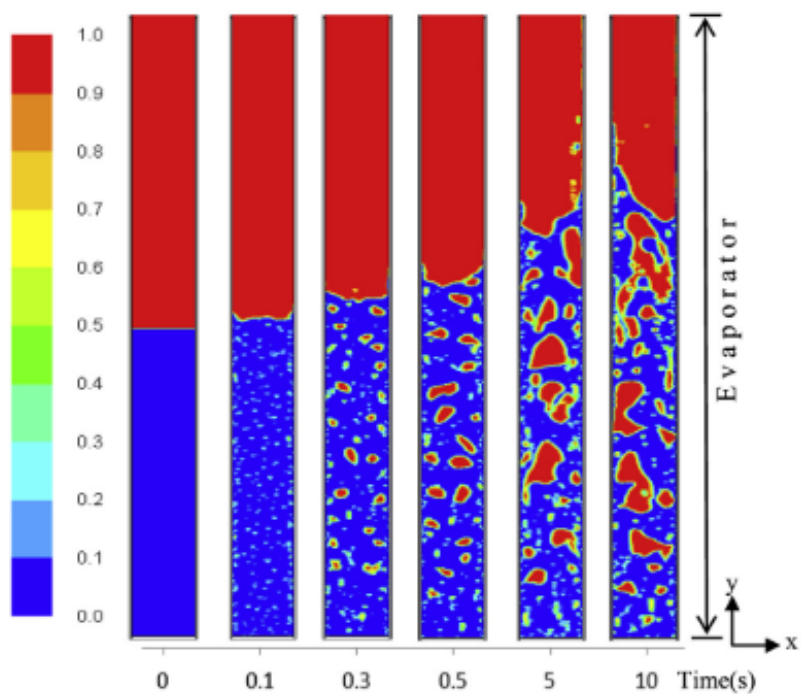

Figure 23: CFD modelling of pool boiling in a thermosyphon, by Fadbl et al. [169]

In terms of temperature distribution, the maximum relative error between the CFD model and the experiments obtain was 13\%. Later, Fadhl et al. [170] similarly modelled a thermosyphon using R134a and R404a as working fluids. The CFD simulation was validated with good agreement between the predicted temperature profiles and experimental data (maximum relative error of $3 \%$ ).

In regard of the preceding introduction made on pool boiling modelling and CFD simulations, it is clear that the progress in this area open new opportunities in the study of boiling. In particular, this tool can be helpful into studying the small-scale mechanisms implied in the bubble dynamic or to investigate boiling conditions that are difficult to observe, such as low gravity pool boiling. As concluded by Dhir [74], it is expected that, in a close future, numerical simulations will become more and more relevant in the study of boiling and phase-change heat transfer. However, up to today, it is not likely that a computational model can provide new and accurate pool boiling heat transfer correlations. Indeed, as reported by Jouhara et al. [153], simulations of boiling and two-phase flow inside a thermosyphon is still at an early stage. Difficulties are currently faced into attesting the reliability and accuracy of a given fluid dynamic simulation. Even if Computational Fluid Dynamic (CFD) modelling is a powerful and promising tool that could lead to new heat transfer correlations, one must be aware that the two-phase phenomena observed are governed by the code implemented and that the results observed reflect the input given and may differ from the reality. Thus, the results of CFD and the phenomena observed in simulations must be analysed with caution and always questioned.

\subsection{Further discussions}

In the presented work reviewing pool-boiling correlations, some factors have not been covered in depth. In some special cases, the pool boiling heat transfer correlation used may need to be adapted to estimate the pool boiling heat transfer coefficient. A brief list of the further parameters that could affect the pool boiling mechanisms is made hereafter:

- $\quad$ Angle and shape of the pool

- Wall thickness and capacity of temporarily storing thermal energy

- Presence of surface treatment 
- $\quad$ Pressure and potential subcooling of the working fluid

- Working fluid used/ Nano fluids/ Liquid metals

- $\quad$ Effect of the pressure and fluid subcooling

- Low gravity conditions

Among others, these parameters may imply a modification of the pool boiling phenomena and adapted heat transfer correlations may be required to estimate the pool boiling resistance of a thermosyphon. Finally, limitations such as the entrainment limit, apparition of dryout and sonic limit must also be taken into account when designing a thermosyphon.

\section{CONCLUSION}

In this paper, the main boiling mechanisms occurring at the evaporator of thermosyphons where only pool boiling takes place have been described. The importance of bubbles in enhancing the nucleate boiling heat transfer coefficients is demonstrated and factors such as the nucleation process, bubble growth, microlayer evaporation, the sensible and latent heat transport coupled with transient conduction occurring at the departure of a bubble from a cavity, the bubble departure diameter and the bubble departure frequency are key factors in improving pool boiling heat transfer and thus the performance of a thermosyphon. To ease the selection of suitable correlations while estimating the bubble departure diameter, frequency and nucleate pool boiling heat transfer coefficient, a critical review has been carried on and the most recommended correlations have been reported in tables. Thus, this paper can be considered as a starting point for thermosyphon users who wants to estimate the nucleate pool boiling resistance of a TPCT. Yet, it also came out that the study of nucleate pool boiling remains not fully understood up to today. In addition, the high discrepancy of boiling experiments is still a main obstacle faced by researchers. Therefore, the intensive development of nucleate boiling correlations is still ongoing. Nevertheless, there is little value in proposing empirical or semi-empirical correlations, as they will probably suit only one special set of boiling data. Instead, focusing on the influence of one parameter in pool boiling and on improving the repeatability of nucleate boiling experiments seems to be a more relevant approach to increase the accuracy of predictive models.

\section{ACKNOWLEDGEMENTS}

This research has been funded by the Innovation and Networks Executive Agency (INEA), European Commission for project PVadapt under Grant Agreement number 818342.

\section{CONFLICT OF INTEREST}

None declared

\section{REFERENCES}

[1] R. S. Gaugler, “Heat transfer device.” Google Patents, 06-Jun-1944.

[2] H. Jouhara, A. Chauhan, T. Nannou, S. Almahmoud, B. Delpech, and L. C. Wrobel, "Heat pipe based systems - Advances and applications," Energy, vol. 128, pp. 729754, Jun. 2017.

[3] K. N. Shukla, “Heat Pipe for Aerospace Applications-An Overview,” J. Electron. Cool. Therm. Control, vol. 05, no. 01, pp. 1-14, 2015.

[4] T. He, C. Mei, and J. P. Longtin, “Thermosyphon-assisted cooling system for refrigeration applications,” Int. J. Refrig., vol. 74, pp. 165-176, Feb. 2017.

[5] P. Gang, F. Huide, Z. Huijuan, and J. Jie, "Performance study and parametric analysis of a novel heat pipe PV/T system,” Energy, vol. 37, no. 1, pp. 384-395, Jan. 2012.

[6] H. N. Chaudhry, B. R. Hughes, and S. A. Ghani, “A review of heat pipe systems for heat recovery and renewable energy applications,” Renew. Sustain. Energy Rev., vol. 16, no. 4, pp. 2249-2259, May 2012. 
[7] W. Qu, "Progress Works of High and Super High Temperature Heat Pipes,” Dev. Heat Transf., p. 13, 2011.

[8] A. A. Eidan, S. E. Najim, and J. M. Jalil, "Experimental and numerical investigation of thermosyphone performance in HVAC system applications," Heat Mass Transf. und Stoffuebertragung, vol. 52, no. 12, pp. 2879-2893, 2016.

[9] H. Mroue, J. B. Ramos, L. C. Wrobel, and H. Jouhara, "Experimental and numerical investigation of an air-to-water heat pipe-based heat exchanger," Appl. Therm. Eng., vol. 78, pp. 339-350, Mar. 2015.

[10] B. Delpech, B. Axcell, and H. Jouhara, "Experimental investigation of a radiative heat pipe for waste heat recovery in a ceramics kiln,” Energy, vol. 170, pp. 636-651, Mar. 2019.

[11] S. Almahmoud and H. Jouhara, "Experimental and theoretical investigation on a radiative flat heat pipe heat exchanger,” Energy, vol. 174, pp. 972-984, May 2019.

[12] H. Jouhara, N. Khordehgah, S. Almahmoud, B. Delpech, A. Chauhan, and S. A. Tassou, "Waste heat recovery technologies and applications," Therm. Sci. Eng. Prog., vol. 6, pp. 268-289, Jun. 2018.

[13] H. Jouhara et al., "The performance of a novel flat heat pipe based thermal and PV/T (photovoltaic and thermal systems) solar collector that can be used as an energy-active building envelope material,” Energy, vol. 108, pp. 148-154, Aug. 2016.

[14] A. Chauhan, J. Trembley, L. C. Wrobel, and H. Jouhara, "Experimental and CFD validation of the thermal performance of a cryogenic batch freezer with the effect of loading,” Energy, vol. 171, pp. 77-94, Mar. 2019.

[15] H. Shabgard, M. J. Allen, N. Sharifi, S. P. Benn, A. Faghri, and T. L. Bergman, “Heat pipe heat exchangers and heat sinks: Opportunities, challenges, applications, analysis, and state of the art,” Int. J. Heat Mass Transf., vol. 89, pp. 138-158, Oct. 2015.

[16] H. Jouhara, "Economic assessment of the benefits of wraparound heat pipes in ventilation processes for hot and humid climates,” Int. J. Low-Carbon Technol., vol. 4, no. 1, pp. 52-60, 2009.

[17] D. Gorenflo, E. Baumhögger, G. Herres, and S. Kotthoff, "Prediction methods for pool boiling heat transfer: A state-of-the-art review,” Int. J. Refrig., vol. 43, pp. 203-226, Jul. 2014.

[18] D. Jafari, P. Di Marco, S. Filippeschi, and A. Franco, “An experimental investigation on the evaporation and condensation heat transfer of two-phase closed thermosyphons,” Exp. Therm. Fluid Sci., vol. 88, pp. 111-123, Nov. 2017.

[19] M. S. El-Genk and H. H. Saber, "Heat transfer correlations for small, uniformly heated liquid pools,” Int. J. Heat Mass Transf., vol. 41, no. 2, pp. 261-274, 1997.

[20] Y. A. Cengel, “Heat Transfer: A Practical Approach,” New York McGraw-Hill, pp. 785-841, 2003.

[21] W. M. Rohsenow Editor et al., "Handbook of heat transfer,” 1998.

[22] ESDU, "Heat pipes - performances of two-phase closed thermosyphons," ESDU 81038, no. November, 1983.

[23] C. L. Tien, “A hydrodynamic model for nucleate pool boiling,” Int. J. Heat Mass Transf., vol. 5, no. 6, pp. 533-540, Jun. 1962. 
[24] V. P. Carey, “Liquid-Vapor Phase-Change Phenomena,” Hemisph. Publ. Corp. DC, 1992.

[25] J. H. Lienhard, "Correlation for the limiting liquid superheat,” Chem. Eng. Sci., vol. 31, no. 9, pp. 847-849, Jan. 1976.

[26] J. H. Lienhard, N. Shamsundar, and P. O. Biney, "Spinodal lines and equations of state: A review,” Nucl. Eng. Des., vol. 95, pp. 297-314, Aug. 1986.

[27] C. T. Avedisian, “The Homogeneous Nucleation Limits of Liquids,” Journal of Physical and Chemical Reference Data, vol. 14, no. 3. pp. 695-729, 1985.

[28] R. Cole, “Boiling Nucleation,” Adv. Heat Transf., vol. 10, pp. 85-166, Jan. 1974.

[29] W. M. Rohsenow, J. P. Hartnett, and E. N. Ganic, Handbook of heat transfer fundamentals. New York, 1985.

[30] G. F. Hewitt, G. L. Shires, and T. R. Bott, Process heat transfer, vol. 113. CRC press Boca Raton, FL, 1994.

[31] C. H. Wang and V. K. Dhir, "On the prediction of active nucleation sites including the effect of surface wettability," in Proceedings of the Engineering Foundation Conference on Pool and External Flow Boiling, 1992, pp. 111-118.

[32] S. G. Bankoff, "Entrapment of gas in the spreading of a liquid over a rough surface," AIChE J., vol. 4, no. 1, pp. 24-26, 1958.

[33] K. A. Zhokhov, "Number of vapour generating centers,” Aerodyn. Heat Transf. Work. Elem. Power Equipment, Leningrad, Russ., pp. 131-135, 1969.

[34] B. B. Mikic and W. M. Rohsenow, "A New Correlation of Pool-Boiling Data Including the Effect of Heating Surface Characteristics,” J. Heat Transfer, vol. 91, no. 2, pp. 245-250, May 1969.

[35] R. L. Mohanty and M. K. Das, “A critical review on bubble dynamics parameters influencing boiling heat transfer,” Renew. Sustain. Energy Rev., vol. 78, pp. 466-494, Oct. 2017.

[36] Lord Rayleigh, "On the pressure developed in a liquid during the collapse of a spherical cavity,” London, Edinburgh, Dublin Philos. Mag. J. Sci., vol. 34, no. 200, pp. 94-98, 1917.

[37] M. S. Plesset and S. A. Zwick, "The Growth of Vapour Bubbles in Superheated Liquid,” J. Appl. Phys., vol. 25, pp. 493-500, 1954.

[38] B. . Mikic, W. . Rohsenow, and P. Griffith, “On bubble growth rates,” Int. J. Heat Mass Transf., vol. 13, no. 4, pp. 657-666, Apr. 1970.

[39] O. Miyatake, I. Tanaka, and N. Lior, “A simple universal equation for bubble growth in pure liquids and binary solutions with a nonvolatile solute," Int. J. Heat Mass Transf., vol. 40, no. 7, pp. 1577-1584, May 1997.

[40] T. G. Theofanous and P. D. Patel, "Universal relations for bubble growth,” Int. J. Heat Mass Transf., vol. 19, no. 4, pp. 425-429, Apr. 1976.

[41] H. K. Forster and N. Zuber, "Growth of a Vapor Bubble in a Superheated Liquid,” J. Appl. Phys., vol. 25, no. 4, pp. 474-478, Apr. 1954.

[42] N. Zuber, “The dynamics of vapor bubbles in nonuniform temperature fields,” Int. $J$. Heat Mass Transf., vol. 2, no. 1-2, pp. 83-98, Mar. 1961. 
[43] B. B. Mikic and W. M. Rohsenow, "Bubble growth rates in non-uniform temperature field,” Prog. Heat Mass Transf., vol. 2, pp. 283-293, 1969.

[44] R. Cole and H. L. Shulman, "Bubble growth rates at high Jakob numbers,” Int. J. Heat Mass Transf., vol. 9, no. 12, pp. 1377-1390, Dec. 1966.

[45] M. G. Cooper, “The microlayer and bubble growth in nucleate pool boiling,” Int. J. Heat Mass Transf., vol. 12, no. 8, pp. 915-933, Aug. 1969.

[46] W. Fritz, "Berechnung des maximalvolumes von dampfblasen,” Phys. Zeitschr, vol. 36, pp. 379-384, 1935.

[47] B. E. Staniszewski, "Nucleate boiling bubble growth and departure,” Cambridge, Mass.: Massachusetts Institute of Technology, Division of Industrial Cooperation, 1959.

[48] E. Ruckenstein, "A physical model for nucleate boiling heat transfer from a horizontal surface,” Bul. Institutului Politeh. Bucuresti Chang. to Bul. Inst. Politeh.'Gheorghe Gheorghiu-Dej'Bucuresti, vol. 23, 1961.

[49] R. Cole and H. L. Shulman, "Bubble departure diameters at subatmospheric pressures,” in Chem. Eng. Prog. Symp. Ser, 1966, vol. 62, no. 64, pp. 6-16.

[50] R. Cole, "Bubble frequencies and departure volumes at subatmospheric pressures," AIChE J., vol. 13, no. 4, pp. 779-783, 1967.

[51] R. Cole and W. M. Rohsenow, "Correlation of bubble departure diameters for boiling of saturated liquids,” in Chem. Eng. Prog. Symp. Ser, 1969, vol. 65, no. 92, pp. 211213.

[52] S. J. D. Van Stralen and W. Zijl, "Fundamental developments in bubble dynamics,” in Proceedings of the sixth international heat transfer conference, Toronto, 1978, vol. 6, pp. 429-450.

[53] V. S. Golorin, B. A. Kol'chugin, and E. A. Zakharova, "Investigation of the mechanism of nucleate boiling of ethyl alcohol and benzene by means of high-speed motion-picture photography,” Heat Transf. Res, vol. 10, no. 4, pp. 79-98, 1978.

[54] S. S. Kutateladze and I. I. Gogonin, "Growth rate and detachment diameter of a vapor bubble in free convection boiling of a saturated liquid,” Teplofiz. Vysok. Temp., vol. 17, pp. 792-797, 1979.

[55] G. Kocamustafaogullari, "Pressure dependence of bubble departure diameter for water,” Int. Commun. Heat Mass Transf., vol. 10, no. 6, pp. 501-509, Nov. 1983.

[56] D. Gorenflo, V. Knabe, and V. Bieling, "Bubble Density on Surfaces With Nucleate Boiling-It's Influence on Heat Transfer and Burnout Heat Fluxes at Elevated Saturation Pressures," in Proceedings of 8th International Heat Transfer Conference, 1986, pp. 1995-2000.

[57] M. K. Jensen and G. J. Memmel, "Evaluation of bubble departure diameter correlations," in Proceedings of the Eighth International Heat Transfer Conference, 1986, vol. 4, pp. 1907-1912.

[58] U. Wenzel and P. Stephan, "Saturated pool boiling and subcooled flow boiling of mixtures at atmospheric pressure.” ResearchSpace@ Auckland, 1992.

[59] L. Z. Zeng, J. F. Klausner, and R. Mei, “A unified model for the prediction of bubble detachment diameters in boiling systems — I. Pool boiling,” Int. J. Heat Mass Transf., 
vol. 36, no. 9, pp. 2261-2270, Jan. 1993.

[60] C. Yang, Y. Wu, X. Yuan, and C. Ma, "Study on bubble dynamics for pool nucleate boiling,” Int. J. Heat Mass Transf., vol. 43, no. 2, pp. 203-208, Jan. 2000.

[61] H. C. Lee, B. Do Oh, S. W. Bae, and M. H. Kim, "Single bubble growth in saturated pool boiling on a constant wall temperature surface,” Int. J. Multiph. Flow, vol. 29, no. 12, pp. 1857-1874, Dec. 2003.

[62] J. Kim and M. H. Kim, "On the departure behaviors of bubble at nucleate pool boiling,” Int. J. Multiph. Flow, vol. 32, no. 10-11, pp. 1269-1286, Oct. 2006.

[63] H. T. Phan, N. Caney, P. Marty, S. Colasson, and J. Gavillet, "Surface wettability control by nanocoating: The effects on pool boiling heat transfer and nucleation mechanism,” Int. J. Heat Mass Transf., vol. 52, no. 23-24, pp. 5459-5471, Nov. 2009.

[64] H. T. Phan, N. Caney, P. Marty, S. Colasson, and J. Gavillet, "A model to predict the effect of contact angle on the bubble departure diameter during heterogeneous boiling,” Int. Commun. Heat Mass Transf., vol. 37, no. 8, pp. 964-969, Oct. 2010.

[65] Y. Nam, E. Aktinol, V. K. Dhir, and Y. S. Ju, "Single bubble dynamics on a superhydrophilic surface with artificial nucleation sites,” Int. J. Heat Mass Transf., vol. 54, no. 7-8, pp. 1572-1577, Mar. 2011.

[66] M. I. Lamas, J. M. S. Jabardo, A. Arce, and P. Fariñas, "Numerical analysis of the bubble detachment diameter in nucleate boiling," in Journal of Physics: Conference Series, 2012, vol. 395, no. 1, p. 12174.

[67] S. Hamzekhani, M. Maniavi Falahieh, and A. Akbari, "Bubble departure diameter in nucleate pool boiling at saturation: Pure liquids and binary mixtures," Int. J. Refrig., vol. 46, pp. 50-58, Oct. 2014.

[68] A. Suszko and M. S. El-Genk, "Saturation boiling of PF-5060 on rough Cu surfaces: Bubbles transient growth, departure diameter and detachment frequency," Int. J. Heat Mass Transf., vol. 91, pp. 363-373, Dec. 2015.

[69] S. Bovard, H. Asadinia, G. Hosseini, and S. A. Alavi Fazel, "Investigation and experimental analysis of the bubble departure diameter in pure liquids on horizontal cylindrical heater,” Heat Mass Transf., vol. 53, no. 4, pp. 1199-1210, 2017.

[70] X. Wang, Z. Wu, J. Wei, and B. Sundén, "Correlations for prediction of the bubble departure radius on smooth flat surface during nucleate pool boiling," Int. J. Heat Mass Transf., vol. 132, pp. 699-714, Apr. 2019.

[71] I. L. Pioro, W. Rohsenow, and S. S. Doerffer, "Nucleate pool-boiling heat transfer. I: Review of parametric effects of boiling surface,” Int. J. Heat Mass Transf., vol. 47, no. 23, pp. 5033-5044, 2004.

[72] J. Du, C. Zhao, and H. Bo, "A modified model for bubble growth rate and bubble departure diameter in nucleate pool boiling covering a wide range of pressures,” Appl. Therm. Eng., vol. 145, pp. 407-415, Dec. 2018.

[73] H. Chen, G. Chen, X. Zou, Y. Yao, and M. Gong, "Experimental investigations on bubble departure diameter and frequency of methane saturated nucleate pool boiling at four different pressures,” Int. J. Heat Mass Transf., vol. 112, pp. 662-675, Sep. 2017.

[74] V. K. Dhir, "Numerical simulations of pool-boiling heat transfer," AIChE J., vol. 47, no. 4, pp. 813-834, 2001. 
[75] W. Gao, J. Qi, X. Yang, J. Zhang, and D. Wu, “Experimental investigation on bubble departure diameter in pool boiling under sub-atmospheric pressure," Int. J. Heat Mass Transf., vol. 134, pp. 933-947, May 2019.

[76] N. Kumar, M. Q. Raza, and R. Raj, "Surfactant aided bubble departure during pool boiling,” Int. J. Therm. Sci., vol. 131, pp. 105-113, Sep. 2018.

[77] P. Stephan and T. Fuchs, "Local heat flow and temperature fluctuations in wall and fluid in nucleate boiling systems," Heat Mass Transf. und Stoffuebertragung, vol. 45, no. 7, pp. 919-928, 2009.

[78] J. G. Collier and J. R. Thome, Convective boiling and condensation. Clarendon Press, 1994.

[79] H. J. Ivey, "Relationships between bubble frequency, departure diameter and rise velocity in nucleate boiling,” Int. J. Heat Mass Transf., vol. 10, no. 8, pp. 1023-1040, Aug. 1967.

[80] M. Jakob and W. Fritz, "Versuche uber den verdampfungsvorgang. Forschung auf dem Gebiete des Ingenieurwesens,” 1931.

[81] M. Jakob and W. Linke, "Heat transfer from a horizontal plate,” Forsch. Geb. Ingenieurwes, vol. 4, p. 434, 1933.

[82] M. Jakob, “Heat Transfer, 1, Chapter 29.” Wiley and Sons, New York, 1949.

[83] F. N. Peebles, "Studies on the motion of gas bubbles in liquid," Chem. Eng. Prog., vol. 49, no. 2, pp. 88-97, 1953.

[84] R. Cole, "A photographic study of pool boiling in the region of the critical heat flux," AIChE J., vol. 6, no. 4, pp. 533-538, 1960.

[85] P. W. McFadden and P. Grassmann, "The relation between bubble frequency and diameter during nucleate pool boiling,” Int. J. Heat Mass Transf., vol. 5, no. 3-4, pp. 169-173, Mar. 1962.

[86] N. Zuber, "Nucleate boiling. The region of isolated bubbles and the similarity with natural convection,” Int. J. Heat Mass Transf., vol. 6, no. 1, pp. 53-78, Jan. 1963.

[87] A. P. Hatton and I. S. Hall, "Photographic study of boiling on prepared surfaces," in Proc. Third Int. Heat Transfer Conf., Chicago, Illinois, USA, Aug. 7-12, 1966, 1966, vol. 4, pp. 24-37.

[88] K. Stephan, Heat transfer in condensation and boiling. Springer, 1992.

[89] T. Kumada, H. Sakashita, and H. Yamagishi, "Pool boiling heat transfer-I. Measurement and semi-empirical relations of detachment frequencies of coalesced bubbles,” Int. J. Heat Mass Transf., vol. 38, no. 6, pp. 969-977, Apr. 1995.

[90] J. H. Kim, S. M. You, and J. Y. Pak, "Effects of heater size and working fluids on nucleate boiling heat transfer," Int. J. Heat Mass Transf., vol. 49, no. 1-2, pp. 122131, Jan. 2006.

[91] H. Sakashita and A. Ono, "Boiling behaviors and critical heat flux on a horizontal plate in saturated pool boiling of water at high pressures,” Int. J. Heat Mass Transf., vol. 52, no. 3-4, pp. 744-750, Jan. 2009.

[92] P. Goel, A. K. Nayak, P. P. Kulkarni, and J. B. Joshi, "Experimental study on bubble departure characteristics in subcooled nucleate pool boiling,” Int. J. Multiph. Flow, vol. 89, pp. 163-176, Mar. 2017. 
[93] K. Nishikawa, Y. Fujita, S. Uchida, and H. Ohta, "Effect of surface configuration on nucleate boiling heat transfer," Int. J. Heat Mass Transf., vol. 27, no. 9, pp. 15591571, 1984.

[94] H. Merte Jr, H. S. Lee, and J. S. Ervin, “Transient nucleate pool boiling in microgravity: Some initial results,” Microgravity Sci. Technol., vol. 7, pp. 173-179, 1994.

[95] R. F. Gaertner, "Photographic study of nucleate pool boiling on a horizontal surface," J. Heat Transfer, vol. 87, no. 1, pp. 17-27, 1965.

[96] S. Nukiyama, "The maximum and minimum values of the heat Q transmitted from metal to boiling water under atmospheric pressure,” Int. J. Heat Mass Transf., vol. 9, no. 12, pp. 1419-1433, 1966.

[97] F. P. Incropera, D. P. DeWitt, T. L. Bergman, and A. S. Lavine, "Fundamentals of Heat and Mass Transfer,” Fundamentals of Heat and Mass Transfer. p. 997, 2007.

[98] H. Jouhara and A. J. Robinson, "Experimental investigation of small diameter twophase closed thermosyphons charged with water, FC-84, FC-77 and FC-3283,” Appl. Therm. Eng., vol. 30, no. 2-3, pp. 201-211, Feb. 2010.

[99] Y. Kim, D. H. Shin, J. S. Kim, S. M. You, and J. Lee, "Boiling and condensation heat transfer of inclined two-phase closed thermosyphon with various filling ratios," Appl. Therm. Eng., vol. 145, pp. 328-342, Dec. 2018.

[100] H. Mroue, J. B. Ramos, L. C. Wrobel, and H. Jouhara, "Performance evaluation of a multi-pass air-to-water thermosyphon-based heat exchanger," Energy, vol. 139, pp. 1243-1260, Nov. 2017.

[101] J. Ramos, A. Chong, and H. Jouhara, "Experimental and numerical investigation of a cross flow air-to-water heat pipe-based heat exchanger used in waste heat recovery," Int. J. Heat Mass Transf., vol. 102, pp. 1267-1281, Nov. 2016.

[102] W. M. Rohsenow, "A method of correlating heat-transfer data for surface boiling of liquids,” 1952.

[103] G. N. Kruzhilin, "Free-convection transfer of heat from a horizontal plate and boiling liquid,” Dokl. AN SSSR (Reports USSR Acad. Sci., vol. 58, no. 8, pp. 1657-1660, 1947.

[104] M. J. McNelly, “A correlation of rates of heat transfer to nucleate boiling of liquids,” J. Imp. Coll. Chem. Eng. Soc, vol. 7, pp. 18-34, 1953.

[105] H. K. Forster and N. Zuber, "Dynamics of vapor bubbles and boiling heat transfer," AIChE J., vol. 1, no. 4, pp. 531-535, 1955.

[106] J. H. Lienhard, “A semi-rational nucleate boiling heat flux correlation,” Int. J. Heat Mass Transf., vol. 6, no. 3, pp. 215-219, Mar. 1963.

[107] I. L. Mostinkski, “Application of the rule of corresponding states for calculation of heat transfer and critical heat flux,” Teploenergetika, vol. 4, no. 1963, pp. 66-71, 1963.

[108] G. N. Danilova, “Correlation of boiling heat transfer data for Freons,” Heat Transf. Res, vol. 2, no. 2, pp. 73-78, 1970.

[109] D. A. Labuntsov, "Heat transfer problems with nucleate boiling of liquids," Therm. Eng.(USSR)(Engl. Transl.), v. 19, no. 9, pp. 21-28, 1973.

[110] H. Imura, H. Kusuda, J.-I. Ogata, T. Miyazaki, and N. Sakamoto, "Heat transfer in two-phase closed-type thermosyphons,” JSME Trans., vol. 45, pp. 712-722, 1979. 
[111] K. Stephan and P. Preusser, "Wrmebergang und maximale wrmestromdichte beim behiterienden binrer und ternrer flussigkeitsgemische,” Chem Ing Tech, 1979.

[112] K. Stephan and M. Abdelsalam, "Heat-transfer correlations for natural convection boiling,” Int. J. Heat Mass Transf., vol. 23, no. 1, pp. 73-87, Jan. 1980.

[113] M. Shiraishi, K. Kikuchi, and T. Yamanishi, "Investigation of heat transfer characteristics of a two-phase closed thermosyphon,” J. Heat Recover. Syst., vol. 1, no. 4, pp. 287-297, Jan. 1981.

[114] K. Bier, J. Schmadl, and D. Gorenflo, "Influence of heat flux and saturation pressure on pool boiling heat transfer of binary mixtures.," VT VERFAHRENSTECHNIK., vol. 16, no. 9, pp. 708-710, 1982.

[115] K. Nishikawa, Y. Fujita, H. Ohta, and S. Hidaka, "Effect of the surface roughness on the nucleate boiling heat transfer over the wide range of pressure," in Heat Transfer, Proceedings of the International Heat Transfer Conference, 1982, pp. 61-66.

[116] M. G. Cooper, "Saturation nucleate pool boiling - A simple correlation,” Inst. Chem. Eng. Symp. Ser., vol. 2.86, pp. 785-793, Jan. 1984.

[117] T. Ueda, T. Miyashita, and P.-H. Chu, Heat transport characteristics of a closed twophase thermosyphon, vol. 54. 1988.

[118] S. S. Kutateladze, "Heat Transfer and Hydrodynamic Resistance: Handbook (in Russian), Chapter 12.7.” Energoatomizdat Publishing House, Moscow, 1990.

[119] U. Groß, "Pool Boiling Heat Transfer inside a Two-Phase Thermosyphon (Correlation of Experimental Data)," in Proc. 9th Int. Heat Transfer Conf., 1990, 1990, vol. 2, pp. 57-62.

[120] D. Gorenflo, P. Sokol, and S. Caplanis, "Pool boiling heat transfer from single plain tubes to various hydrocarbons,” Int. J. Refrig., vol. 13, no. 5, pp. 286-292, Sep. 1990.

[121] F. Kaminaga, Y. Okamoto, T. Suzuki, and T. Ma, "Study on boiling heat transfer correlation in a closed two-phase thermosyphon," in Proceedings of the 8th International Heat Pipe Conference, Beijing, 1992.

[122] W. Leiner, "Heat transfer by nucleate pool boiling-general correlation based on thermodynamic similarity,” Int. J. Heat Mass Transf., vol. 37, no. 5, pp. 763-769, Mar. 1994.

[123] W. Leiner and D. Gorenflo, "Methods of predicting the boiling curve and a new equation based on thermodynamic similarity,” Pool Extern. Flow Boil., pp. 99-103, 1992.

[124] F. M. Chowdhury, F. Kaminaga, K. Goto, and K. Matsumura, "Boiling heat transfer in a small diameter tube below atmospheric pressure on a natural circulation condition," J. Japan Assoc. Heat Pipe, vol. 16, no. 1997, pp. 14-16, 1997.

[125] T. Kiatsiriroat, A. Nuntaphan, and J. Tiansuwan, "Thermal performance enhancement of thermosyphon heat pipe with binary working fluids,” Exp. Heat Transf., vol. 13, no. 2, pp. 137-152, 2000.

[126] G. Ribatski and J. M. S. Jabardo, "Experimental study of nucleate boiling of halocarbon refrigerants on cylindrical surfaces,” Int. J. Heat Mass Transf., vol. 46, no. 23, pp. 4439-4451, Nov. 2003.

[127] I. L. Pioro, W. Rohsenow, and S. S. Doerffer, "Nucleate pool-boiling heat transfer. II: 
assessment of prediction methods,” Int. J. Heat Mass Transf., vol. 47, no. 23, pp. 5045-5057, Nov. 2004.

[128] Y. J. Park, H. K. Kang, and C. J. Kim, "Heat transfer characteristics of a two-phase closed thermosyphon to the fill charge ratio," Int. J. Heat Mass Transf., vol. 45, no. 23, pp. 4655-4661, Nov. 2002.

[129] S. H. Noie, "Heat transfer characteristics of a two-phase closed thermosyphon,” Appl. Therm. Eng., vol. 25, no. 4, pp. 495-506, Mar. 2005.

[130] W. Guo and D. W. Nutter, "An experimental study of axial conduction through a thermosyphon pipe wall,” Appl. Therm. Eng., vol. 29, no. 17-18, pp. 3536-3541, Dec. 2009.

[131] D. Jafari, A. Franco, S. Filippeschi, and P. Di Marco, "Two-phase closed thermosyphons: A review of studies and solar applications,” Renew. Sustain. Energy Rev., vol. 53, pp. 575-593, Jan. 2016.

[132] F. M. Chowdhury and Fumito Kaminaga, "Boiling heat transfer characteristics of R113 in a vertical small diameter tube under natural circulation condition,” Int. J. Heat Mass Transf., vol. 45, no. 24, pp. 4823-4829, Nov. 2002.

[133] A. Nakano, M. Shiraishi, M. Nishio, and M. Murakami, “An experimental study of heat transfer characteristics of a two-phase nitrogen thermosyphon over a large dynamic range operation,” Cryogenics (Guildf)., vol. 38, no. 12, pp. 1259-1266, Dec. 1998.

[134] D. Jafari, S. Filippeschi, A. Franco, and P. Di Marco, "Unsteady experimental and numerical analysis of a two-phase closed thermosyphon at different filling ratios," Exp. Therm. Fluid Sci., vol. 81, pp. 164-174, Feb. 2017.

[135] B. J. Jones, J. P. McHale, and S. V. Garimella, "The Influence of Surface Roughness on Nucleate Pool Boiling Heat Transfer,” J. Heat Transfer, vol. 131, no. 12, p. 121009, 2009.

[136] D. Gorenflo, “Pool Boiling: VDI-Heat Atlas,” VDI-Verlag, Dusseldorf, Ger., 1993.

[137] P. A. Kew and K. Cornwell, "Correlations for the prediction of boiling heat transfer in small-diameter channels,” Appl. Therm. Eng., vol. 17, no. 8-10, pp. 705-715, Aug. 1997.

[138] K. Smith, A. J. Robinson, and R. Kempers, "Confinement and vapour production rate influences in closed two-phase reflux thermosyphons Part B: Heat transfer,” Int. J. Heat Mass Transf., vol. 120, pp. 1241-1254, May 2018.

[139] G. B. Wallis, “One-dimensional two-phase flow,” 1969.

[140] I. I. Chernobylskii and I. M. Tananaiko, "Heat exchange during boiling of liquids in narrow annular tubes,” Sov. physics-technical Phys., vol. 1, no. 10, pp. 2244-2249, 1956.

[141] E. Ishibashi and K. Nishikawa, “Saturated boiling heat transfer in narrow spaces,” Int. J. Heat Mass Transf., vol. 12, no. 8, pp. 863-893, Aug. 1969.

[142] S.-C. Yao and Y. Chang, "Pool boiling heat transfer in a confined space," Int. J. Heat Mass Transf., vol. 26, no. 6, pp. 841-848, Jun. 1983.

[143] Y. Fujita, H. Ohta, S. Uchida, and K. Nishikawa, "Nucleate boiling heat transfer and critical heat flux in narrow space between rectangular surfaces,” Int. J. Heat Mass 
Transf., vol. 31, no. 2, pp. 229-239, Feb. 1988.

[144] G. M. Lazarek and S. H. Black, "Evaporative heat transfer, pressure drop and critical heat flux in a small vertical tube with R-113,” Int. J. Heat Mass Transf., vol. 25, no. 7, pp. 945-960, 1982.

[145] H. Kuncoro, Y. F. Rao, and K. Fukuda, "An experimental study on the mechanism of geysering in a closed two-phase thermosyphon,” Int. J. Multiph. Flow, vol. 21, no. 6, pp. 1243-1252, Nov. 1995.

[146] M. R. S. Emami, S. H. Noie, M. Khoshnoodi, M. T. H. Mosavian, and A. Kianifar, "Investigation of geyser boiling phenomenon in a two-phase closed thermosyphon," Heat Transf. Eng., vol. 30, no. 5, pp. 408-415, 2009.

[147] K. Negishi and T. Sawada, "Heat transfer performance of an inclined two-phase closed thermosyphon,” Int. J. Heat Mass Transf., vol. 26, no. 8, pp. 1207-1213, Aug. 1983.

[148] A. Kujawska, B. Zajaczkowski, L. M. Wilde, and M. H. Buschmann, "Geyser boiling in a thermosyphon with nanofluids and surfactant solution,” Int. J. Therm. Sci., vol. 139, no. October 2018, pp. 195-216, 2019.

[149] S. K. Morgan and H. F. Brady, "Elimination of the geysering effect in missiles," in Advances in Cryogenic Engineering, Springer, 1962, pp. 206-213.

[150] S. H. Noie, M. R. S. Emami, and M. Khoshnoodi, "Effect of inclination angle and filling ratio on thermal performance of a two-phase closed thermosyphon under normal operating conditions," Heat Transf. Eng., vol. 28, no. 4, pp. 365-371, 2007.

[151] T. F. Lin, W. T. Lin, Y. L. Tsay, J. C. Wu, and R. J. Shyu, "Experimental investigation of geyser boiling in an annular two-phase closed thermosyphon,” Int. J. Heat Mass Transf., vol. 38, no. 2, pp. 295-307, Jan. 1995.

[152] X. Wang, Y. Wang, H. Chen, and Y. Zhu, “A combined CFD/visualization investigation of heat transfer behaviors during geyser boiling in two-phase closed thermosyphon,” Int. J. Heat Mass Transf., vol. 121, pp. 703-714, Jun. 2018.

[153] H. Jouhara, B. Fadhl, and L. C. Wrobel, "Three-dimensional CFD simulation of geyser boiling in a two-phase closed thermosyphon,” Int. J. Hydrogen Energy, vol. 41, no. 37, pp. 16463-16476, Oct. 2016.

[154] K. Smith, R. Kempers, and A. J. Robinson, "Confinement and vapour production rate influences in closed two-phase reflux thermosyphons Part A: Flow regimes,” Int. J. Heat Mass Transf., vol. 119, pp. 907-921, Apr. 2018.

[155] I. Khazaee, R. Hosseini, and S. H. Noie, "Experimental investigation of effective parameters and correlation of geyser boiling in a two-phase closed thermosyphon," Appl. Therm. Eng., vol. 30, no. 5, pp. 406-412, Apr. 2010.

[156] G. Xia, W. Wang, L. Cheng, and D. Ma, "Visualization study on the instabilities of phase-change heat transfer in a flat two-phase closed thermosyphon," Appl. Therm. Eng., vol. 116, pp. 392-405, Apr. 2017.

[157] A. Kujawska, B. Zajaczkowski, L. M. Wilde, and M. H. Buschmann, "Geyser boiling in a thermosyphon with nanofluids and surfactant solution,” Int. J. Therm. Sci., vol. 139, no. February, pp. 195-216, 2019.

[158] Y. Lee and U. Mital, “A two-phase closed thermosyphon,” Int. J. Heat Mass Transf., vol. 15, no. 9, pp. 1695-1707, Sep. 1972. 
[159] C. Casarosa, E. Latrofa, and A. Shelginski, "The geyser effect in a two-phase thermosyphon,” Int. J. Heat Mass Transf., vol. 26, no. 6, pp. 933-941, Jun. 1983.

[160] P. Stephan and J. Hammer, “A new model for nucleate boiling heat transfer,” vol. 30, pp. 119-125, 1994.

[161] S. C. K. De Schepper, G. J. Heynderickx, and G. B. Marin, "Modeling the evaporation of a hydrocarbon feedstock in the convection section of a steam cracker," Comput. Chem. Eng., vol. 33, no. 1, pp. 122-132, Jan. 2009.

[162] S. C. K. De Schepper, G. J. Heynderickx, and G. B. Marin, "CFD modeling of all gasliquid and vapor-liquid flow regimes predicted by the Baker chart," Chem. Eng. J., vol. 138, no. 1-3, pp. 349-357, May 2008.

[163] J. . Brackbill, D. . Kothe, and C. Zemach, "A continuum method for modeling surface tension,” J. Comput. Phys., vol. 100, no. 2, pp. 335-354, Jun. 1992.

[164] J. Legierski, B. Wie, cek, and G. de Mey, "Measurements and simulations of transient characteristics of heat pipes,” Microelectron. Reliab., vol. 46, no. 1, pp. 109-115, Jan. 2006.

[165] M. Zhang, Z. Liu, G. Ma, and S. Cheng, "Numerical simulation and experimental verification of a flat two-phase thermosyphon," Energy Convers. Manag., vol. 50, no. 4, pp. 1095-1100, Apr. 2009.

[166] A. Alizadehdakhel, M. Rahimi, and A. A. Alsairafi, "CFD modeling of flow and heat transfer in a thermosyphon," Int. Commun. Heat Mass Transf., vol. 37, no. 3, pp. 312318, Mar. 2010.

[167] A. S. Annamalai and V. Ramalingam, "Experimental investigation and computational fluid dynamics analysis of a air cooled condenser heat pipe,” Therm. Sci., vol. 15, no. 3, pp. 759-772, 2011.

[168] Z. Lin, S. Wang, R. Shirakashi, and L. Winston Zhang, "Simulation of a miniature oscillating heat pipe in bottom heating mode using CFD with unsteady modeling," Int. J. Heat Mass Transf., vol. 57, no. 2, pp. 642-656, Feb. 2013.

[169] B. Fadhl, L. C. Wrobel, and H. Jouhara, "Numerical modelling of the temperature distribution in a two-phase closed thermosyphon,” Appl. Therm. Eng., vol. 60, no. 1-2, pp. 122-131, Oct. 2013.

[170] B. Fadhl, L. C. Wrobel, and H. Jouhara, "CFD modelling of a two-phase closed thermosyphon charged with R134a and R404a,” Appl. Therm. Eng., vol. 78, pp. 482490, Mar. 2015. 